This item was submitted to Loughborough's Research Repository by the author.

Items in Figshare are protected by copyright, with all rights reserved, unless otherwise indicated.

\title{
On the acoustic response of a generic gas turbine fuel injector passage
}

\section{PLEASE CITE THE PUBLISHED VERSION}

https://doi.org/10.1016/j.jsv.2019.01.043

\section{PUBLISHER}

(c) Elsevier

\section{VERSION}

AM (Accepted Manuscript)

\section{PUBLISHER STATEMENT}

This paper was accepted for publication in the journal Journal of Sound and Vibration and the definitive published version is available at https://doi.org/10.1016/j.jsv.2019.01.043.

\section{LICENCE}

CC BY-NC-ND 4.0

\section{REPOSITORY RECORD}

Su, Jialin, Andrew Garmory, and Jon Carrotte. 2019. "On the Acoustic Response of a Generic Gas Turbine Fuel Injector Passage". Loughborough University. https://hdl.handle.net/2134/36727. 


\title{
On the Acoustic Response of a Generic Gas Turbine Fuel Injector Passage
}

\author{
J. Su ${ }^{\mathrm{a}, *}$, A. Garmory ${ }^{\mathrm{a}}$, J.F. Carrotte ${ }^{\mathrm{a}}$ \\ ${ }^{a}$ Department of Aeronautical and Automotive Engineering, Loughborough University, \\ Loughborough, LE11 3TU, United Kingdom
}

\begin{abstract}
A current trend in the design of modern aero engines is the transition towards leaner combustion as a solution to satisfy increasingly stringent emission regulations. Lean combustion systems are often more susceptible to thermoacoustic instability and the fuel injector can play a critical role. This paper presents an analytical study on the unsteady air flow through a generic injector passage in response to incident acoustic waves. The injector passage is represented by a simplified geometry which comprises the main geometrical passage features. The unsteady flow through the passage is obtained by combining the elemental solutions for different parts of the passage. This enables the transfer impedance of the injector passage to be determined and the effects of different design parameters on the sensitivity of the air flow to acoustic perturbations to be examined. The convective wave associated with the unsteady swirl vane wakes is also visited and compared with the results from the numerical simulations obtained in previous works. In addition to helping derive design practices for injector passages from the perspective of thermoacoustic instability, the current analysis can also be applied as a preliminary design tool to assess the acoustic characteristics for an injector passage of the axial swirler type.
\end{abstract}

Keywords:

fuel injector, thermoacoustic instability, acoustic wave, transfer impedance, swirl vane wake

\footnotetext{
*Corresponding author

Email address: j.su@lboro.ac.uk (J. Su )
} 


\section{Nomenclature}

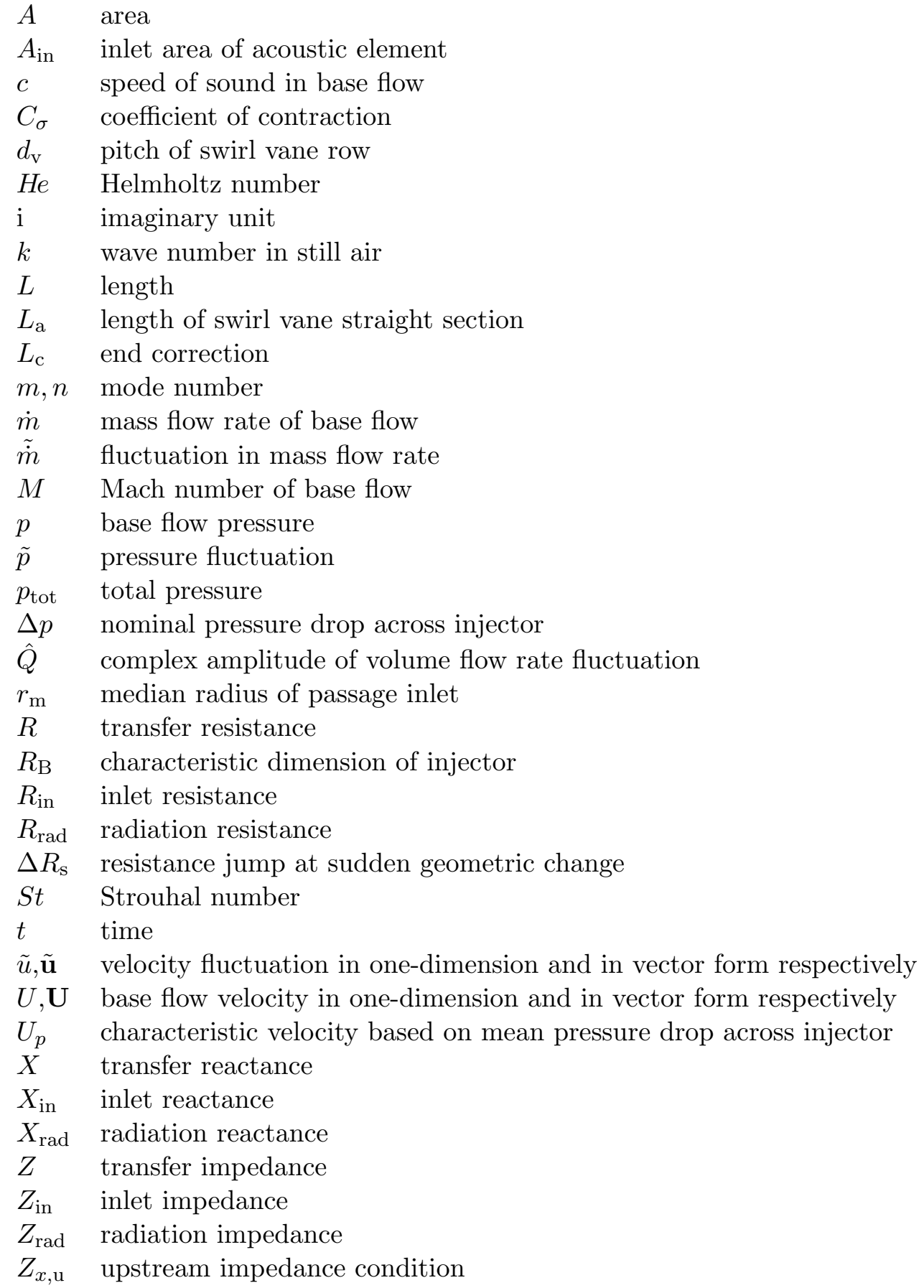


$Z_{x} \quad$ acoustic impedance

$\eta_{\mathrm{a}} \quad$ coefficient of aerodynamic efficiency of injector passage

$\theta \quad$ swirl vane turning angle

$\rho \quad$ base flow density

$\tilde{\rho} \quad$ density fluctuation

$\rho_{0} \quad$ air density in plenum

$\tilde{\phi} \quad$ fluctuation in velocity potential

$\omega \quad$ angular frequency

\section{Accents}

$\sim$ fluctuation in time
$\quad$ complex amplitude of fluctuation

\section{Introduction}

Depending on the application either liquid or gaseous fuel is introduced into the combustion system of modern gas turbines through fuel injectors. These injectors are designed to enable the combustion system to fulfil a number of operability requirements which include the need to avoid thermoacoustic instabilities over the engine operating range. However, the drive to reduce pollutant formation has resulted in a transition towards lean combustion which makes the system more prone to such instabilities. Extensive research on this phenomenon has been reported in the literature, e.g. $[1,2,3,4,5,6,7,8,9]$. In the thermoacoustic feedback loop the pressure waves generated in the combustion process, typically by unsteady heat release, interact with the fuel injector and induce fluctuations in the air flow passing through it and entering the combustion zone. As a result further unsteadiness in the combustion process can be provoked. At the same time, fluctuations in the injector flow can lead to perturbations in stoichiometry of the flow being delivered to the heat release region, as well as affecting the atomisation process when liquid fuel is used. These factors also contribute to the unsteadiness of the combustion process. The role played by the fuel injector in the thermoacoustic instability is of particular significance, and more so for lean combustion systems, in which the majority of the combustor air flow passes through the fuel injector.

The injectors used within modern gas turbines have evolved into highly complex geometries displaying a variety of different styles. However, the main 
considerations in injector design are generally focused around generating flow conditions that enhance fuel/air mixing and produce a flow region dedicated to flame stabilisation. Typically modern fuel injectors consist of one or more passages through which the air flow passes whilst tangential momentum (i.e swirl) is imparted to it. This is usually accomplished via turning vanes which creates a downstream recirculation region within which the flame is stabilised. As the flow passes through the injector gaseous or liquid fuel is introduced into the airstream. Numerous studies (e.g. $[10,11,12,13,14,15])$ have been carried out to understand the various aerodynamic processes and flow field features generated by the injector such as vortex breakdown, precessing vortex core (PVC), and central toroidal recirculation zone (CTRZ), etc. The findings from these works are in general most beneficial for optimising injector design with the aim of improving combustion efficiency and reducing the formation of pollutants. However, also of concern is the unsteady flow generated by the fuel injector due to incident acoustic perturbations on account of its effect on thermoacoustic instability. Studies on both non-reacting (e.g. $[16,17,18,19,20])$ and reactng (e.g. $[5,21,22,23,24,25])$ scenarios have been reported.

The stability of the whole combustion system has been conveniently evaluated using low order network models such as those introduced by Dowling and Stow [26] and Paschereit et al. [27]. In such a model each acoustic element can be characterised with an acoustic transfer matrix (e.g. [28, 29, $30,31]$ ). Given the acoustic boundary conditions of the system, the acoustic impedance at any point in the acoustic network can be calculated from the transfer matrices of the acoustic elements. When the base flow in a system is supplied by atmosphere from upstream, the acoustic impedance at a point in the acoustic network is defined as $Z_{x}=-\hat{p} / \hat{Q}$ observed from downstream relative to the air supply. Here $\hat{p}$ and $\hat{Q}$ are the complex amplitudes of the fluctuations in pressure and volume flux respectively. The acoustic impedance describes the stiffness of the air flow response to the pressure fluctuation imposed at the corresponding location of the system. Alternative to transfer matrix, the passive acoustic elements in a system can often also be represented by their transfer impedances (e.g. [32, 33, 34]) when an acoustic circuit type of approach is adopted. The transfer impedance of an acoustically compact element is generally calculated as the ratio between the fluctuation in pressure difference across the acoustic element and the fluctuation in volume flux through it. It is essentially equal to the acoustic impedance change across the element. Considering this and the variation of 
unsteady volume flux within a non-compact acoustic element, the transfer impedance of the injector is defined in a generalised sense here to match the acoustic impedance change across the injector:

$$
Z=Z_{x, \mathrm{~d}}-Z_{x, \mathrm{u}}=\frac{\hat{p}_{\mathrm{u}}}{\hat{Q}_{\mathrm{u}}}-\frac{\hat{p}_{\mathrm{d}}}{\hat{Q}_{\mathrm{d}}}=R+\mathrm{i} X
$$

where ' $u$ ' and ' $d$ ' denote the flow conditions upstream and downstream of the injector respectively.

The transfer impedance, $Z$, has real (transfer resistance, $R$ ) and imaginary (transfer reactance, $X$ ) components. It can also be derived from the transfer matrix of the injector. As will be discussed in Section 3.2.2, the influence of the upstream impedance condition, $Z_{x, \mathrm{u}}=-\hat{p}_{\mathrm{u}} / \hat{Q}_{\mathrm{u}}$, on the transfer impedance defined by Eq. (1) is estimated to be of second order of combinations of small non-dimensional parameters including Helmholtz number, Mach number and the cross-section area ratio between the injector passage and upstream plenum. For a typical gas turbine setup this influence is shown to be small as far as the current study is concerned. Meanwhile, according to its definition $Z$ is not a function of the transfer impedances of the acoustic elements downstream. Based on the above, the transfer impedance can be practically considered as a local property of the injector. For low Helmholtz numbers (e.g. less than 0.5), the difference between $\hat{Q}_{\mathrm{u}}$ and $\hat{Q}_{\mathrm{d}}$ is small and the inverse of $Z$ indicates the sensitivity of air flow rate through the injector with respect to the fluctuation in pressure difference across the injector. When $Z_{x, \mathrm{u}}$ has been determined with the acoustic boundary condition and elements upstream of the injector, the acoustic impedance at the injector exit is obtained by combining $Z_{x, \mathrm{u}}$ and the injector transfer impedance directly. For the frequency range relevant to thermoacoustic instability, the injector transfer impedance is an important constituent of the acoustic impedance at the injector exit.

As previously described the incident acoustic waves generated by the unsteady heat release from the combustion process can lead to fluctuation in the air flow through the injector. It is obvious that a large injector transfer impedance is desirable for combustion stability. In this aspect, characterising a fuel injector with the transfer impedance provides a convenient way to define a design target for fuel injector from the thermoacoustic perspective based on a largely local property of the injector. The transfer resistance is generally dominated by the loss associated with the mean/base air flow through the injector. Considering the amount of air flow through the fuel 
injector in a lean combustion system, it is crucial that the efficiency of the injector air flow under nominal operating conditions be not compromised for an injector design intended to increase transfer resistance. Meanwhile, the transfer reactance mainly corresponds to the inertia of air flow within the fuel injector and, in general, increases with the injector length. This, however, will be constrained by the physical dimension of the whole combustion system.

For a low-order thermoacoustic network model another important component is the flame transfer/describing function (FTF/FDF) which correlates the unsteady air flow through the fuel injector with the fluctuation in flame heat release. For swirl stabilised flames, which are widely adopted in modern gas turbine engines, the combustion process is also affected by the circumferential component of the injector air flow. This can often be analysed through the non-dimensional swirl number $[35,36]$ parameter. It is well recognised that acoustic perturbation also induces a circumferential velocity fluctuation downstream of the swirl vanes, which is convected at the local air flow velocity $[18,37,38,39]$. Indeed a convective wave had been earlier identified in a study on sound transmission through a blade row [40], but has to date mostly been ignored by acousticians because it does not induce any pressure fluctuation (c.f. Section 5). It can, however, have a significant effect on the combustion process, and therefore needs to be included in the analysis of thermoacoustic instability. Based on the corresponding FTF/FDF, Palies et al. [38] and Candel et al. [39] showed that the effect of circumferential velocity fluctuation on the unsteady heat release can interact either constructively or destructively with that due to the axial velocity fluctuation depending on the excitation frequency.

On account of the general geometric complexity of the fuel injector, accurate characterisation, for example, by transfer matrix or transfer impedance, of the acoustic response of injector air flow is often carried out by experimental or numerical means (e.g. [19, 21, 28, 30, 31, 38]). On the other hand, a widely adopted approach in analytical studies is to simplify the injector as a constant-sectioned duct which is defined by a characteristic area and an effective length, plus a loss coefficient (e.g. [3, 26, 29]). This has been extended to using a series of connected ducts for a more generalised representation of the fuel injector $[30,41]$. Despite extensive research efforts on the acoustic response of injector air flows, a large part of the studies available in literature have been focused upon extracting/predicting the acoustic characteristics of the injector as a lumped acoustic element and their subsequent application for stability analysis of the combustion system. There are rela- 
tively limited results published with regard to generic design practices that optimise the acoustic characteristics of fuel injector for the benefit of combustion stability. The aforementioned duct approximations for fuel injectors generally suggest that contracting the injector exit cross-section increases acoustic damping and hence the transfer resistance, whilst lengthening the injector results in higher transfer reactance. Nevertheless, in a practical design the former modification will inflict higher loss on the base flow and the latter is limited by the combustion system geometries. Instead of increasing the transfer impedance, manipulating the convective time lags of the fluctuations of various combustion-controlling quantities, such as equivalence ratio and swirl number, was also discussed [38, 42]. The corresponding injector design changes will, however, to a large extent depend on the actual combustion process which is at the same also determined by the other components of the combustion system.

As lean combustion technologies gain more popularity in future gas turbine design, it is of importance to gain more insight into the acoustic response of the injector air flow and continue exploring generic and applicable injector design practices that help alleviate thermoacoustic instability. This paper presents an analytical study on the unsteady air flow in a generic injector passage triggered by incident acoustic waves. At first the passage geometry is divided into various zones and the corresponding unsteady flow solutions for individual zones are obtained separately. These elemental solutions are subsequently assembled to form the complete solution for the whole passage. The sensitivity of air flow within the injector passage with respect to acoustic perturbation is investigated mainly based on the transfer impedance of injector passage calculated from the unsteady flow solution. By examining the relevant physics and the effects of different design parameters of the injector passage on its transfer impedance, this study helps shed light on design practices that reduce the sensitivity of the injector air flow to incident acoustic waves (or, equivalently, increase the transfer impedance). In addition, the convective wave associated with the circumferential velocity fluctuation is revisited, adding more physical insight to its influence on the unsteady air flow inside the injector passage. Provided with suitable mean flow parameters, the analysis can be readily applied at a preliminary design stage for an injector passage of the generic type considered in this paper. 


\section{Experimental and Numerical Studies}

The acoustically excited unsteady flow in a generic single-passage injector configuration has been investigated in a combined experimental and numerical study reported by $\mathrm{Su}$ et al. [19]. The experiments were conducted with an impedance tube style setup using the multi-microphone technique. The test facility is isothermal, operating at atmospheric conditions. A depiction is given in Fig. 1. A centrifugal fan was used to generate a suitable pressure drop across the fuel injector such that the mean baseline flow was at a Mach number comparable with engine conditions. The flow was produced by atmospheric air from the upper plenum passing through the fuel injector, before continuing down a straight square-sectioned duct and entering the exhaust plenum, where it was extracted back to atmosphere. In this study the mean pressure drop, $\Delta p$, across the injector is approximately $3 \%$ of the pressure in the upstream plenum. The mean mass flow rate, $\dot{m}$, through the injector can be measured with a metering orifice deployed in the duct. For the single-passage injector the total aerodynamic loss, $\Delta p_{\text {tot }}$ (i.e. loss in total pressure), incurred by the injector passage on the air flow can be expressed as below in terms of a coefficient of aerodynamic efficiency, $\eta_{\mathrm{a}}$, defined as the ratio between the actual and ideal mass flow rate:

$$
\Delta p_{\mathrm{tot}}=\Delta p\left(1-\eta_{\mathrm{a}}^{2}\right), \quad \eta_{\mathrm{a}}=\frac{\dot{m}}{\rho_{0} A_{\mathrm{out}} U_{p} \cos \theta}, \quad U_{p}=\sqrt{\frac{2 \Delta p}{\rho_{0}}}
$$

where $\rho_{0}$ is the air density in the upstream plenum, $A_{\text {out }}$ is the the outlet area of the injector passage and $\theta$ is the swirl vane turning angle. Generally $\eta_{\mathrm{a}}$ is a characteristic property of the injector passage, which is mostly independent of $\Delta p$. The generic axial swirler type of annular passage considered in this work is representative of the main passage designs commonly found in the fuel injectors of medium and large lean combustion gas turbine engines. Such type of injector passage is in general significantly larger in dimension than its counterpart for rich burn injectors, typically at least twice as large in radius and more than three times as long in length.

Acoustic measurements were conducted without the metering orifice. Plane acoustic waves were created by two loud speakers located near the downstream end of the duct, with the waves travelling upstream towards the injector and against the base flow direction. In the experiments, the engine frequency range of concern is scaled down to atmospheric conditions by 
matching the Strouhal number defined as:

$$
S t=\omega R_{\mathrm{B}} / U_{p}
$$

where $\omega$ is the angular frequency and $R_{\mathrm{B}}$ is a characteristic dimension of the injector. Four Kulite dynamic pressure transducers were installed at different axial locations along the duct to measure the unsteady pressure downstream of the injector. A detailed description of the test facility, instrumentation and data processing methodology are given in [43] and [44]. From the unsteady pressure measured by the Kulites, the incident and reflected acoustic waves were reconstructed based on the multi-microphone technique, whereby the pressure and velocity fluctuations at the injector exit, i.e. $\hat{p}_{\mathrm{d}}$ and $\hat{u}_{\mathrm{d}}$, can be determined.

In the current experimental setup, the pressure fluctuation in the upstream plenum is nearly zero except for a small region in the vicinity of the injector inlet. This provides a datum upstream condition of $\hat{p}_{\mathrm{u}} \approx 0$ and $Z_{x, \mathrm{u}} \approx 0$, so that the measured transfer impedance is essentially $Z=-\hat{p}_{\mathrm{d}} / \hat{Q}_{\mathrm{d}}$. In an industrial gas turbine the injector inlet is open to an upstream chamber of finite size, which imposes an upstream impedance condition $Z_{x, \mathrm{u}} \neq 0$. In Section 3.2.2 the influence of this impedance condition on $Z$, as defined by Eq. (1), is estimated to be small for the current study. Meanwhile, finiteness of the upstream chamber results in negligible change in the transfer resistance but a lower transfer reactance compared to that under the infinite plenum condition. As to be discussed in Section 3.2.1 this is due to a reduced end correction at the passage inlet. For the single passage considered here the percentage of such a change in the transfer reactance is estimated to be small and does not affect the following analysis on the internal geometric parameters of injector passage.

In [19] numerical simulations with computational fluid dynamics (CFD) were also reported based on the experimental scenario. The computational domain is illustrated in Fig. 2. It consists of a duct section whose length is more than ten times the injector diameter, the full injector geometry and a hemispherical artificial plenum. A schematic of the single-passage injector is included in Fig. 2. The commercial package ICEM- CFD was used to generate the computational grid (also shown in Fig. 2). The CFD calculations were performed with the open-source programme OpenFOAM [45] (version 2.1.1). The approach of unsteady Reynolds-averaged Navier-Stokes simulation (URANS) was adopted with the $k-\omega$ shear-stress-transport (SST) turbu- 
lence model. The compressible unsteady flow was solved using the pressurebased algorithm of Pressure Implicit with Splitting of Operator (PISO) introduced by Issa [46]. At the same time, suitable acoustic boundary conditions were derived from the Euler equations and applied in the simulations to match the corresponding experimental conditions. Specifically plane pressure waves of specified frequencies and amplitudes are injected at the outlet of the duct section, whilst the hemispheric inlet surface of the plenum is set to be non-reflective. Details of the numerical methodology and simulation parameters are given in [19]. An example of the instantaneous flow field is given in Fig. 3 showing the axial velocity contour and the flow streamlines in the injector centre cross-plane. In the plot $L$ is a characteristic injector length scale and $U_{p}$ is the velocity scale introduced in Eq. (2). From the simulation results the injector transfer impedance was determined in the same manner as in the experiments by application of the multi-microphone method to the pressure data at the same axial locations as those of the Kulites in the experiments.

The results obtained from the experiments and the CFD simulations respectively are reproduced in Fig. 4. The Strouhal number is defined with the median radius, $r_{\mathrm{m}}$, of the passage inlet as the characteristic dimension (i.e. $\left.R_{\mathrm{B}}=r_{\mathrm{m}}\right)$. The passage inlet area, $A_{\mathrm{in}}$, is used as the reference area for non-dimensionalisation of $Z$. The frequency range considered in this work corresponds to $0.18<S t<0.2$, which includes the frequency span most relevant to thermoacoustic instability plus a small margin at both the low and high frequency ends. The accuracy of numerical simulations is verified by the agreement between the experimental measurements and CFD results. Thus having validated the CFD methodology one can interrogate these CFD solutions for details of the acoustically perturbed unsteady flow field inside the injector passage which is difficult to access by experimental means. The experimental and CFD studies together provide the data for comparison with the analysis to be presented in the following sections of the paper. Thereby this analysis can be applied to identify the effects of various geometric features of the injector passage on its response characteristics to incident acoustic waves.

\section{Propagation of Acoustic Waves through Injector Passage}

In this work, an analytical study is carried out for the acoustically perturbed unsteady flow inside the single-passage injector configuration shown 
in Fig. 2.

\subsection{Formulation of Problem}

When the disturbances are significantly smaller than the base flow states, the acoustically perturbed unsteady flow can be described by the linearised continuity, momentum (i.e. Navier-Stokes equations) and energy equations around the base flow conditions. The set of equations is completed with the equation of state, for which the ideal gas law is assumed. In high Reynolds number flows, the viscous effect is usually confined to certain compact regions (e.g. boundary layers and vortex sheets). As a result, for the bulk flow the Navier-Stokes equations may be reduced to the Euler equations. In this section the acoustic propagation within the injector passage is considered by solving the linear wave equations, complemented by the assumption of concentrated hydrodynamic losses, for a simplified geometry that includes the passage design parameters to be studied.

\subsubsection{Simplified Geometry for Analytical Study}

For the passage dimensions and frequency range considered in the present work no persistent radial or circumferential modes can be established inside the passage. On account of an outer-inner radius ratio close to one, the radial variation of the flow field is ignored. The annular passage can be approximated by a straight flow channel between two bounding walls. The span of the channel is equal to the median line circumference at the passage inlet. The distance between the bounding walls at an axial location is defined such that the channel cross-section area matches that of the actual passage geometry at the same location. By virtue of periodicity the flow channel can be extended spanwise to form an infinite self-repeating domain. But the transfer impedance will be calculated only over one span. The mid-plane section of the channel is represented by the simplified geometry illustrated in Fig. 5 including notations of the main passage geometric parameters. The swirl vanes are assumed to be of zero thickness and their shape is simplified to a circular arc connected to a straight tangent line segment. The pitch of swirl vane row is measured at the swirl vane midspan. The entire passage is divided into four zones along the axial direction. Zone I extends from just downstream of the bell-mouthed inlet up to the leading edge of the swirl vane

row. Zone II is the front turning sections of the pathways formed between neighbouring swirl vanes, whilst the rear straight sections of the pathways 
are designated as zone III. The remaining part of the passage (from the swirl vane trailing edges to the passage exit) is zone IV.

\subsubsection{Hydrodynamic Loss at Sudden Geometrical Change}

A concentrated hydrodynamic loss usually arises at the location of a geometrical discontinuity, such as a sudden change of cross-section area. At the steady state (i.e. base flow) this loss (in total pressure) across the discontinuity can be expressed in terms of a coefficient of contraction, $C_{\sigma}$, as follows:

$$
\Delta p_{\text {tot }}=\frac{1}{2} \rho_{\mathrm{d}} U_{\mathrm{d}}^{2}\left(\frac{1}{C_{\sigma}^{2}}-1\right)
$$

where $\rho_{\mathrm{d}}$ and $U_{\mathrm{d}}$ are the base flow density and velocity downstream of the geometrical change respectively. The lossless condition is $C_{\sigma}=1$. On account of its compactness, such a region of sudden geometrical change is modelled with a jump in transfer resistance when calculating the transfer impedance of the whole injector passage. Assuming that its variation with frequency is small, this resistance jump is effectively equal to its value at the quasi-steady state (i.e. $\omega \approx 0$ ), $\Delta R_{\mathrm{s}}$, given as:

$$
\Delta R_{\mathrm{s}}=\dot{m}\left[\left(\frac{1}{C_{\sigma} A_{\mathrm{d}}}\right)^{2}-\left(\frac{1}{A_{\mathrm{u}}}\right)^{2}\right]
$$

where $A_{\mathrm{u}}$ and $A_{\mathrm{d}}$ are the cross-section areas upstream and downstream of the geometrical change respectively. When Eq. (5) is applied to an orifice connecting two plenums, $\Delta R_{\mathrm{s}} \approx \dot{m} /\left(C_{\sigma} A\right)^{2}$ at the orifice inlet and $\Delta R_{\mathrm{s}} \approx 0$ at the exit assuming negligible pressure recovery. Their sum is equivalent to the corresponding term accounting for the acoustic resistance of the orifice in the works by Luong et al. [47] and Bellucci et al. [48]. In this case $C_{\sigma}$ at the orifice inlet is essentially the discharge coefficient.

\subsubsection{Hydrodynamic Loss in Turbulent Boundary Layer}

In a wall-bounded internal flow such as that within the injector passage, the effect of viscosity is most prominent in the boundary layers (laminar or turbulent). Given the conditions of low Mach number flows (typically $M<0.2$ ) and adiabatic walls (negligible heat transfer) this effect is mainly of a hydrodynamic nature and its contribution to the transfer impedance can be assessed at the incompressible limit. To this end, the incompressible oscillating straight channel flows at representative base flow conditions within 
the passage are considered for an estimation of the viscous effect on the unsteady passage flow.

The base flow Reynolds number based on the passage annular gap width suggests that it belongs to the turbulent regime. Using Prandtl's mixing length hypothesis, Howe [49] derived an analytical expression for the attenuation of acoustic waves in parallel turbulent channel flows. More recently Weng et al. [50] reconsidered the same problem using a Reynolds stress transport model in the Reynolds-averaged Navier-Stokes (RANS) equations. Both theories predicted that, for the Reynolds number and frequency range considered in the present paper, the variation of transfer resistance with respect to frequency is small due to turbulence relaxation (i.e. delay in the response of turbulence to acoustic perturbation), whilst the boundary layer effect on the transfer reactance is negligible compared to the inertia of the flow itself. The flow through the passage is not actually parallel to the passage walls as the boundary layers are developing on both walls. Using URANS CFD simulations, $\mathrm{Su}$ [51] investigated the incompressible oscillating flows with developing turbulent boundary layers for a two-dimensional straight channel whose length is equal to the passage length and width equal to the passage annular gap width. Two flow speeds were considered, corresponding the flow conditions in the passage upstream and downstream of the swirl vane row respectively. The results obtained led to a judgement on the boundary layer effects concurring with that derived from the theories by Howe and Weng et al.

Based on the above discussion, the viscous effect appears to the unsteady passage flow as mostly a distributed hydrodynamic loss. Such a loss is generally difficult to separate from the total flow loss. To reduce the complexity of the problem for the current analytical study, the contribution of viscous effect to the transfer impedance is simplified to a concentrated hydrodynamic

loss and merged with that occurring at a more distinguishable geometrical location within the passage where the highest loss is observed.

\subsubsection{Governing Equations of Inviscid Unsteady Flows}

The linearised continuity and Euler equations for the unsteady flow within the injector passage read:

$$
\begin{gathered}
\frac{\partial \tilde{\rho}}{\partial t}+\nabla \cdot(\rho \tilde{\mathbf{u}}+\tilde{\rho} \mathbf{U})=0 \\
\rho \frac{\partial \tilde{\mathbf{u}}}{\partial t}+\rho(\mathbf{U} \cdot \nabla \tilde{\mathbf{u}}+\tilde{\mathbf{u}} \cdot \nabla \mathbf{U})+\tilde{\rho} \mathbf{U} \cdot \nabla \mathbf{U}+\nabla \tilde{p}=0
\end{gathered}
$$


where $\mathbf{U}$ is the base flow velocity, and $\tilde{\rho}$ and $\tilde{\mathbf{u}}$ are the fluctuations in density and velocity respectively. In the absence of any entropy waves, the inviscid and adiabatic assumptions reduce the equations of energy conservation and state to the following relation between $\tilde{p}$ and $\tilde{\rho}$ [52, pp. 120]:

$$
\tilde{p} / \tilde{\rho}=c^{2}
$$

where $c$ is the speed of sound in the base flow. In parallel with the inviscid condition, it is further assumed that any vorticity is confined to compact regions of singularities that occupy only infinitesimal volumes of the flow field (e.g. a vortex sheet). Thereby the unsteady flow field is irrotational and can be expressed in terms of a fluctuating velocity potential, $\tilde{\phi}$, such that $\tilde{\mathbf{u}}=\nabla \tilde{\phi}$.

The base flow states in the injector passage need to be specified for Eqs. (6)-(8). The process of establishing the base flow is outlined in Appendix A. The base flow parameter required as the external input is the coefficient of aerodynamic efficiency, $\eta_{\mathrm{a}}$. It can be obtained from experiments, CFD, or in a preliminary design stage through empirical estimations. In the current analysis the value derived from the base flow CFD simulation is adopted. By minimising the uncertainties in the base flow conditions, this enables a direct comparison of the following analytical calculations with the CFD simulations of the acoustically excited unsteady flow. With the flow details accessible from the CFD results such a comparison helps gain more insight of the unsteady flow. The air flow within the injector passage is assumed to be lossless except at certain locations of geometrical discontinuity, where concentrated hydrodynamic losses are introduced. Different ways of distributing the total loss along the passage were attempted in [51]. The changes in the results, particularly the total passage transfer impedance, were found to be negligible. From the base flow CFD result the profile loss at the swirl vane leading edges is identified as the largest contributor to the total loss for the flow inside the injector passage. As a practical choice, the total loss is simplified to a hydrodynamic loss concentrated at the swirl vane leading edges, which can be calculated with $\Delta p$ and $\eta_{\mathrm{a}}$, c.f. Eq. (2).

\subsection{Solutions for Representative Constitutive Geometries}

Starting from Eqs. (6)-(8), different formulations are applied for the unsteady flow in the different passage regions illustrated in Fig. 5. The individual solutions for the representative geometrical features comprising the 
passage are presented here as the building elements for the whole passage solution. The transfer impedance of the passage inlet consists of the radiation impedance and a resistive component associated with area contraction. Its estimation is described in Section 3.2.1. In zone I the passage cross-section area can generally vary along the axial direction and the unsteady flow is solved using a quasi one-dimensional approach as presented in Section 3.2.2. It is noted that for the injector passage considered here the cross-section area is constant in zone I and a closed-form solution of the unsteady flow in this zone is available. Based on the argument to be given in Section 3.2.3, the unsteady flow in zone II is also calculated with the quasi one-dimensional formulation. In zones III and IV combined, the problem is presented as the interaction between a flat plate array and incident acoustic waves approaching from downstream. In zone IV the variation of passage cross-section area normal to the axial direction is small in the current analysis. Hence the passage cross-section area in zone IV is assumed to be constant and equal to that in zone III such that the base flow for Eqs. (6)-(8) is uniform in these two zones. A closed-form solution of the unsteady flow in zones III and IV can be obtained as to be described in Section 3.2.4.

\subsubsection{Radiation and Inlet Impedances}

With the injector passage open to an ample upstream plenum, there arises a radiation impedance at the passage inlet. In the experiment the injector is mounted in a plate of a width more than eight times the median radius of the passage inlet, which can be treated effectively as an infinite flange. Therefore the radiation impedance of the passage inlet is initially estimated following an approach similar to that described in $[52, \S 7.5(\mathrm{a})]$ and $[53, \S 302]$ for a flanged circular inlet but adapted here for a flanged annular inlet. The fluctuating flow at the inlet cross-section of the passage is modelled as an annular piston vibrating along its axis with a fluctuating velocity $\tilde{u}$. The annular piston is represented by a collection of infinitesimal baffled point sources of strength, $\tilde{u} \mathrm{~d} A$, where $\mathrm{d} A$ is the elemental area occupied by a baffled point source on the piston. An illustration of the baffled point source and the annular piston is given in Fig. 6, where $r_{1}$ and $r_{2}$ are the inner and outer radii of the annular passage inlet respectively.

The total force induced by the baffled point sources upon the annular piston itself is given by the double integral below [52, pp. 185]:

$$
\mathrm{f}=\mathrm{i} \frac{\rho \omega \tilde{u}}{2 \pi} \iint \frac{e^{-\mathrm{i} k z}}{z} \mathrm{~d} A \mathrm{~d} A^{\prime}
$$


where $k=\omega / c$ is the wave number in still air and $z$ is the distance between the elemental areas $\mathrm{d} A$ and $\mathrm{d} A^{\prime}$. In Eq. (9) both integration regions $A$ and $A^{\prime}$ refer to the surface of the annular piston. The base flow states are taken at the passage inlet, i.e. $\rho=\rho_{\text {in }}$ and $c=c_{\text {in }}$. Following the procedure described in $[52, \S 7.5(\mathrm{a})]$, the double integration of Eq. (9) is evaluted in the form below:

$$
\begin{aligned}
& \mathrm{f}=\rho_{\text {in }} c_{\text {in }} \tilde{u}\left[\mathrm{f}_{\mathrm{c}}\left(r_{2}\right)-\mathrm{f}_{\mathrm{c}}\left(r_{1}\right)-4 \mathrm{i} \int_{r_{1}}^{r_{2}} \int_{-\arccos \left(\frac{r_{1}}{r}\right)}^{\arccos \left(\frac{r_{1}}{r}\right)} e^{-\mathrm{i} k r \cos \Phi} \sin \left(k r_{\sigma}\right) \mathrm{d} \Phi \mathrm{d} r\right] \\
& \mathrm{f}_{\mathrm{c}}(r)=\pi r^{2}\left[1-\frac{J_{1}(2 k r)}{k r}+\mathrm{i} \frac{H_{1}(2 k r)}{k r}\right], \quad r_{\sigma}=\sqrt{r_{1}^{2}-r^{2} \sin ^{2} \Phi}
\end{aligned}
$$

where $J_{1}$ is the first order Bessel function of the first kind and $H_{1}$ is the first order Struve function. Once $\mathrm{f}$ is calculated, the radiation impedance is obtained accordingly as:

$$
Z_{\mathrm{rad}}=\frac{\mathrm{f} / A}{A \tilde{u}}=\frac{\mathrm{f}}{\left[\pi\left(r_{2}^{2}-r_{1}^{2}\right)\right]^{2} \tilde{u}}=R_{\mathrm{rad}}+\mathrm{i} X_{\mathrm{rad}}
$$

The radiation impedance of the passage inlet calculated as above for different frequencies is plotted in Fig. 7. The frequency is expressed as the dimensionless Helmholtz number $H e_{(r)}=k r_{\mathrm{m}}$ where $r_{\mathrm{m}}=\left(r_{1}+r_{2}\right) / 2$ is the median radius of the passage inlet. The frequencies of interest in the current study correspond to $0.05<k r_{\mathrm{m}}<0.25$.

The radiation impedance can also be expressed in terms of a length end correction, $L_{\mathrm{c}}$, and a pressure reflection coefficient, $\left|R_{p}\right|$, as:

$$
\begin{aligned}
Z_{\mathrm{rad}}=R_{\mathrm{rad}}+\mathrm{i} X_{\mathrm{rad}} & =\frac{\rho_{\mathrm{in}} c_{\mathrm{in}}}{A_{\text {in }}} \cdot \frac{1-\left|R_{p}\right| e^{-\mathrm{i} 2 k L_{\mathrm{c}}}}{1+\left|R_{p}\right| e^{-\mathrm{i} 2 k L_{\mathrm{c}}}} \\
& \approx \frac{\rho_{\text {in }} c_{\text {in }}}{A_{\text {in }}} \cdot \frac{1-\left|R_{p}\right|}{1+\left|R_{p}\right|}+\mathrm{i} \frac{\rho_{\text {in }} \omega L_{\mathrm{c}}}{A_{\text {in }}} \quad\left(k L_{\mathrm{c}} \ll 1\right)
\end{aligned}
$$

The calculation presented so far for the radiation impedance has not taken into account the presence of the bellmouth at the passage inlet. It was shown by Selamet et al. [54] that an inlet bellmouth is expected to increase $L_{\mathrm{c}}$. In the current analysis, such an increase in $L_{\mathrm{c}}$ is estimated by interpolation from the plots given in [54] using the ratio between the bellmouth radius, $r_{\mathrm{b}}$, and the annulus half width, $h_{\mathrm{a}}=\left(r_{2}-r_{1}\right) / 2$, in place of the ratio between the 
bellmouth radius and the circular duct radius. This produces an end correction change of $\delta L_{\mathrm{c}} \approx 0.3 h_{\mathrm{a}}$ for the geometry considered here. Meanwhile, in the low frequency range the change in $\left|R_{p}\right|$ caused by the bellmouth is shown to be small in [54]. Thus the influence of bellmouth on $R_{\text {rad }}$ can be assumed to be negligible. Accordingly the radiation impedance calculated with Eq. (11) is supplemented by the additional term, $\mathrm{i} \rho_{\mathrm{in}} \omega \delta L_{\mathrm{c}} / A_{\mathrm{in}}$.

In addition to the radiation impedance, the transfer impedance of the passage inlet also includes a resistive component, $R_{\mathrm{s}, \mathrm{in}}$, that arises due to the area change (contraction). As discussed in Section 3.1.2 this is modelled with $R_{\mathrm{s}, \text { in }}=\dot{m} /\left(C_{\sigma} A_{\text {in }}\right)^{2}$, given by Eq. (5) with $A_{\mathrm{u}}=\infty$ and $A_{\mathrm{d}}=A_{\text {in }}$. Combining it with the corrected radiation impedance, the transfer impedance for the bellmouth inlet of the single injector passage considered here is predicted as:

$$
Z_{\text {in }}=R_{\text {in }}+\mathrm{i} X_{\text {in }}=R_{\text {s,in }}+Z_{\text {rad }}+\mathrm{i} \frac{\rho_{\text {in }} \omega \delta L_{\mathrm{c}}}{A_{\text {in }}}
$$

For $R_{\mathrm{s}, \mathrm{in}}, \dot{m}$ is obtained from $\eta_{\mathrm{a}}$ and the given mean pressure drop $\Delta p$. As aforementioned the CFD-calculated value of $\eta_{\mathrm{a}}$ is used here and the loss at the passage inlet is assumed to be negligible. A comparison of $Z_{\text {in }}$ estimated with Eq. (13) and that calculated from CFD simulations is presented in Fig. 8. The same normalisations as those in Fig. 4 are applied.

The estimated $R_{\text {in }}$ is lower than that calculated with CFD. This is basically due to the assumption $C_{\sigma}=1$. The actual $C_{\sigma}$ can be found when provided with the base flow pressure at the passage inlet, which is available from the CFD result but not from experiments. The corrected inlet resistance calculated with the actual $C_{\sigma}$ is also produced in Fig. 8 and shows an improved match with the CFD values. Nonetheless, $C_{\sigma}$ is in general close to one for a bellmouth, and as indicated in Section 3.1.4 separating the inlet loss from the total loss within the passage only results in a negligible change in the prediction of total passage transfer impedance. To minimise the external input for the current analysis the assumption $C_{\sigma}=1$ is retained for the passage inletand the inlet loss is combined into the concentrated hydrodynamic loss reflected by $\eta_{\mathrm{a}}$. On the other hand, the inlet reactance is well predicted by analytical means.

The calculation of $Z_{\text {rad }}$ in this section for a flanged annular aperture matches the current experimental setup and is valid when the upstream plenum is sufficiently large. In an industrial gas turbine setup (particularly in aerospace application) the injector passage is usually open to an upstream chamber of limited volume. Following the work by Ingard [55] and Yang and 
Morgans [56], the radiation impedance should be substituted by a transfer reactance representing an end correction lower than that corresponding to the infinite plenum scenario. In [55] Ingard also adopted the vibrating piston approximation for evaluation of the end correction of a flanged aperture open to a rectangular or circular chamber. This can be easily adapted for an annular opening by changing the integration limits; for example, one has for a square-sectioned chamber:

$$
L_{\mathrm{c}}=\frac{16 \pi}{a^{2}} \sum_{m}^{\prime} \sum_{n}^{\prime} \frac{\left[r_{2} J_{1}\left(\alpha_{m n} r_{2}\right)-r_{1} J_{1}\left(\alpha_{m n} r_{1}\right)\right]^{2}}{\alpha_{m n}^{3}\left(r_{2}^{2}-r_{1}^{2}\right)}
$$

where $a$ is the side of the chamber and $\alpha_{m n}=2 \pi \sqrt{m^{2}+n^{2}} / a$. The prime on the summation sign indicates that the term of $m=n=0$ is excluded. For the frequency range considered in the current work, the inlet impedance in a finite upstream chamber can be estimated accordingly as:

$$
Z_{\text {in }}=R_{\mathrm{s}, \text { in }}+\mathrm{i} \frac{\rho_{\text {in }} \omega\left(L_{\mathrm{c}}+\delta L_{\mathrm{c}}\right)}{A_{\text {in }}}
$$

It is noted that when $a \rightarrow \infty$ the end correction approaches that in an infinite plenum. However, for such a case a large number of terms are needed in Eq. (14) for the result to converge and the approach based on Eqs. (9) to (11) is preferred when an infinite plenum is present. If Eq. (14) is used for estimation of $L_{\mathrm{c}}$ of the current passage inlet geometry in a medium to large aero-engine setup and $\delta L_{\mathrm{c}}$ is assumed unchanged, the resultant decrease in $X_{\text {in }}$ compared to the infinite plenum scenario is typically no more than $40 \%$ of that shown in Fig. 13 for the frequency range considered here. In Section 3.3 it will be demonstrated that such a change is small relative to the total passage reactance. The finiteness of the upstream chamber cross-section also leads to a lower value of $R_{\mathrm{s}, \text { in }}$ compared to the infinite plenum scenario. However, the difference is usually negligible for a general gas turbine setup as $\Delta R_{\mathrm{s}}$ is a function of the square of cross-section area ratio.

\subsubsection{Quasi One-Dimensional Inviscid Unsteady Flow}

When the variation in the cross-section of a duct is continuous and higher order transverse modes are absent, the following quasi one-dimensional equations can be derived from Eqs. (6) and (7) together with the adiabatic relation Eq. (8) for approximation of the unsteady axial inviscid flows through the duct:

$$
\frac{\mathrm{d} \hat{\dot{m}}}{\mathrm{~d} x}=-\mathrm{i} \frac{\omega A}{c^{2}} \hat{p}
$$




$$
\left(1-M^{2}\right) A \frac{\mathrm{d} \hat{p}}{\mathrm{~d} x}=-\left(2 \frac{\mathrm{d} U}{\mathrm{~d} x}+\mathrm{i} \omega\right) \hat{\dot{m}}+\left[\frac{\mathrm{d}\left(M^{2} A\right)}{\mathrm{d} x}+\mathrm{i} \frac{2 \omega U A}{c^{2}}\right] \hat{p}
$$

where $\tilde{\dot{m}}$ is the fluctuation in mass flow rate, given to the first order as:

$$
\hat{\dot{m}}=\dot{m}\left(\frac{\hat{u}}{U}+\frac{\hat{p}}{\rho c^{2}}\right)=\left(-\frac{\rho}{Z_{x}}+\frac{\dot{m}}{\rho c^{2}}\right) \hat{p}
$$

In Eqs. (16) and (17) the duct cross-section area, $A$, can vary with $x$. When the pressure fluctuation, $\hat{p}_{\text {in }}$, and acoustic impedance, $Z_{x, \text { in }}$, at the duct inlet are specified, the corresponding mass flow fluctuation, $\hat{\dot{m}}_{\mathrm{in}}$, is determined by Eq. (18). The pressure and mass flow fluctuations at the duct outlet can then be obtained by solving the first order ordinary differential equation (ODE) system of Eqs. (16) and (17) with $\hat{p}_{\text {in }}$ and $\hat{\dot{m}}_{\text {in }}$ as the initial conditions. Thereafter the acoustic impedance at the duct outlet, $Z_{x, \text { out }}$, is calculated using Eq. (18). With the duct inlet defined at $x=0$, the upstream impedance condition is specified as $Z_{x, \mathrm{u}}=R_{x, \mathrm{u}}+\mathrm{i} X_{x, \mathrm{u}}$ at $x=0^{-}$. The acoustic impedance at the duct inlet is $Z_{x, \text { in }}=Z_{x, \mathrm{u}}+Z_{\text {in }}$ at $x=0^{+}$when there is a sudden geometric change across $x=0$. An example of the inlet impedance $Z_{\text {in }}$ is that described in Section 3.2.1. The duct transfer impedance is given as $Z=Z_{x, \text { out }}-Z_{x, \mathrm{u}}$. Here $Z_{\text {in }}$ is included in the duct transfer impedance based on the argument that the control volume used for the definition of Eq. (1) should enclose the duct inlet. It is noted that $Z$ depends on $Z_{x \text {,in }}$ only but not $\hat{p}_{\text {in }}$ because Eqs. (16) and (17) are linear. Indeed one can derive from Eqs. (16) and (17) the first order ODEs for $Z_{x}$ as well as $R$ and $X$, which are given in Appendix B by Eqs. (B.1), (B.2) and (B.3) respectively. To calculate $Z$ alone it is sufficient to solve Eq. (B.1) or Eqs. (B.2) and (B.3), but to determine the quasi one-dimensional unsteady flow $(\hat{p}, \hat{\dot{m}}$ and $\hat{u})$ Eqs. (16) and (17) need to solved. There is no general closed-form solution for Eqs. (16) and (17) or Eqs. (B.1), (B.2) and (B.3) except for certain special cases; for example, a straight duct of uniform cross-section. For the calculations in the current work the linear ODE system of Eqs. (16) and (17) is used, which is of simpler forms than Eqs. (B.1), (B.2) and (B.3) and can be numerically integrated to any practical accuracy efficiently.

In view of the base flow condition and the frequency range considered in the current study, the transfer impedance for a duct of length $L$ is examined at small Mach number $M$ and Helmholtz numbers $H e_{(L)}=k L$ and $H e=$ $k\left(L+L_{\mathrm{c}}\right)$. Here $M, H e_{(L)}$ and $H e$ are of similar orders. For such an analysis it is more convenient to consider an asymptotic form of Eqs. (B.2) and 
(B.3) to be derived below with representative $Z_{x, \mathrm{u}}$ and $Z_{\text {in }}$. As indicated in Section 2, upstream of the injector in a gas turbine is generally a chamber of finite volume. A typical upstream condition for this chamber in turn is a choked inlet [26]. The upstream impedance observed by the injector is usually dominated by a reactance $X_{x, \mathrm{u}} \propto \cot \left(k L_{\mathrm{p}}\right)$ in a one-dimensional setting, where $L_{\mathrm{p}}$ is a characteristic length of the chamber. For the frequency range of interest here, $\frac{X_{x, \mathrm{u}} A}{\rho c}$ is negative and typically of the order of the cross-section area ratio, $\beta=A / A_{\mathrm{p}}$, where $A_{\mathrm{p}}$ is a characteristic area of the chamber crosssection. On the other hand, $R_{x, \mathrm{u}}$ is usually negligible, for example, compared to $R_{\text {in }}$. From Section 3.2.1 $\frac{R_{\mathrm{in}} A}{\rho c}$ is typically $O(M)$ and $\frac{X_{\mathrm{in}} A}{\rho c}$ is $O\left(k L_{\mathrm{c}}\right)$.

The leading order of duct transfer impedance is determined by integrals of the terms in the right hand side (RHS) of Eqs. (B.2) and (B.3) that do not depend on $R$ and $X$ themselves or $Z_{x, \mathrm{u}}$. The leading-order terms of the transfer resistance and transfer reactance, $R_{\mathrm{o}}$ and $X_{\mathrm{o}}$, are hence identified as:

$$
R_{\mathrm{o}}=R_{\text {in }}+\frac{\dot{m}}{A^{2}}-\frac{\dot{m}}{A_{\text {in }}^{2}}, \quad X_{\mathrm{o}}=\int^{x} \frac{\rho \omega}{\left(1-M^{2}\right) A} \mathrm{~d} x+X_{\text {in }}
$$

$R_{\mathrm{o}}+\mathrm{i} X_{\mathrm{o}}$ gives the exact transfer impedance of an incompressible flow at the limit of $M \rightarrow 0$ and $H e \rightarrow 0$. For a compressible flow, $\frac{R_{0} A}{\rho c}$ is $O(M)$ and $\frac{X_{0} A}{\rho c}$ is $O(H e)$, which are also the leading orders for $\frac{R A}{\rho c}$ and $\frac{X A}{\rho c}$ respectively. For a leading-order analysis it is desirable to decouple the transfer resistance and transfer reactance equations from each other so that they can be examined separately. To this end $X$ appearing in the RHS of Eq. (B.2) is substituted with $X_{\mathrm{o}}$, and $R$ in the RHS of Eq. (B.3) with $R_{\mathrm{o}}$. Additionally, the nonlinear term $X^{2}$ in Eq. (B.3) is replaced by $X_{\mathrm{o}} X$ which is linear. It is further assumed that $R_{x, \mathrm{u}}$ is negligible. Thereupon one arrives at the following equations:

$$
\begin{aligned}
\frac{\mathrm{d} R}{\mathrm{~d} x}=\dot{m} \frac{\mathrm{d}}{\mathrm{d} x}\left(\frac{1}{A^{2}}\right)-2 \frac{\mathrm{d} M^{2}}{\mathrm{~d} x} R+\frac{2 k}{1-M^{2}} \cdot \frac{\left(X_{x, \mathrm{u}}+X_{\mathrm{o}}\right) A}{\rho c} \cdot R \\
\frac{\mathrm{d} X}{\mathrm{~d} x}=\frac{\rho \omega}{\left(1-M^{2}\right) A}\left[1-\left(\frac{R_{\mathrm{o}} A}{\rho c}\right)^{2}+\left(\frac{X_{x, \mathrm{u}} A}{\rho c}\right)^{2}\right]-2 \frac{\mathrm{d} M^{2}}{\mathrm{~d} x}\left(X_{x, \mathrm{u}}+X\right) \\
+\frac{k}{1-M^{2}} \cdot \frac{\left(2 X_{x, \mathrm{u}}+X_{\mathrm{o}}\right) A}{\rho c} \cdot X
\end{aligned}
$$

Same as Eqs. (B.2) and (B.3), the initial conditions for Eqs. (20) and (21) are $R=R_{\text {in }}$ and $X=X_{\text {in }}$ respectively at $x=0$. Based on the discussion above on $Z_{x, \mathrm{u}}$ it is identified from Eq. (20) that relative to $R_{\mathrm{o}}$ the influence of 
$Z_{x, \mathrm{u}}$ on $R$ is $O\left(H e_{(L)} \cdot \beta\right)$ for the frequency range of interest here. On $X$ the influence of $Z_{x, \mathrm{u}}$ is $O\left(H e_{(L)} \cdot \beta, \beta^{2}, M^{2} \beta / H e\right)$ relative to $X_{\mathrm{o}}$. The influence of $Z_{x, \mathrm{u}}$ is therefore of second order in terms of combinations of the small scaling parameters, $M, \beta, H e_{(L)}$ and $H e$. For a single injector passage this influence is generally small considering the relevant values of these parameters.

In Eq. (21) the dominant term on the RHS is $\rho \omega / A\left(1-M^{2}\right)$, integrating to $X_{\mathrm{o}}$. With respect to this leading-order term the relative orders of the terms containing $X_{x, \mathrm{u}}$ have been discussed above and the remaining higher order terms are $O\left(M^{2}, H e^{2}\right)$ compared to the leading-order term. Considering the relative orders between the terms in Eq. (21), maximising $X$ can be largely achieved by maximising $X_{\mathrm{o}}$. By observation of the form of $X_{\mathrm{o}}$ it can be deduced that when the inlet and outlet areas as well as length of the duct are fixed, the transfer reactance of the duct can be increased by reducing the cross-section area of the duct portion between duct inlet and outlet. In Eq. (20) the first term on the RHS integrates to $R_{0}$. The second term can be approximated to the leading order of $M^{2}$ by $-2 R_{\mathrm{o}} \frac{\mathrm{d} M^{2}}{\mathrm{~d} x}$, which can be expressed as a complete integral in terms of $M^{2}$. Its integration is therefore mainly dependent upon the duct inlet and outlet conditions, and the influence of area variation in between is small. The last term on the RHS of Eq. (20) reflects the amplification of transfer resistance due to non-compactness, which results in the growth of transfer resistance with frequency. To the leading order this term is proportional to $\omega M\left(X_{x, \mathrm{u}}+X_{\mathrm{o}}\right)$. For the frequency range of interest here $X_{x, \mathrm{u}}+X_{\mathrm{o}}$ increases with $x$ and is negative only within a small interval of the integration range $[0, L]$. Since contracting the duct portion between inlet and outlet increases $X_{\mathrm{o}}$ (and therefore $X_{x, \mathrm{u}}+X_{\mathrm{o}}$ ), it reduces the $x$-interval of negative $X_{x, \mathrm{u}}+X_{\mathrm{o}}$ at the same time. Meanwhile, decreasing $A$ also lead to higher $M$. Based on the above, reducing the crosssection area of the duct portion between duct inlet and outlet promotes the growth of transfer resistance with frequency. Eqs. (20) and (21) can be solved analytically. However, the solutions are lengthy and do not provide more information than the equations themselves as far as the influence of the relevant parameters, i.e. $Z_{x, \mathrm{u}}$ and $A$, is concerned. For conciseness they are given in Appendix B without further discussion.

In a practical problem, the duct external dimensions, i.e. $A_{\text {in }}, A_{\text {out }}$ (outlet area), and $L$, are often constrained by factors such as base flow loss and geometrical confinement, etc. The above discussion on duct transfer impedance suggests that when $A_{\text {in }}, A_{\text {out }}$, and $L$ are fixed, $Z$ is increased by reducing the cross-section area of the duct portion between inlet and outlet. This may 
be illustrated by applying Eqs. (16) and (17) to the three duct geometries depicted in Fig. 9, namely ducts with a contraction, a uniform cross-section and a bulge respectively. The cross-section areas at the duct inlet and outlet are fixed to that of the passage inlet, and the duct length is similar to that of the injector passage. For duct (a) in Fig. 9 the cross-section area decreases to $0.5 A_{\text {in }}$ in the central half of the duct. In contrast, the cross-section area is expanded to $1.5 A_{\text {in }}$ at the same part of duct (c). A half cosine function is adopted for smooth transition of the cross-section areas in both ducts. At all the three duct inlets, the base flow states are the same as at the injector passage inlet and the inlet impedance $\left(Z_{\text {in }}\right)$ as calculated in Section 3.2.1 is applied as a representative duct inlet impedance. The upstream impedance condition, $Z_{x, \mathrm{u}}$, is defined based on the upstream chamber used by Stow and Dowling in [3], which is subject to the choked inlet condition and of the dimensions $L_{\mathrm{p}}=0.1 \mathrm{~m}$ and $A_{\mathrm{p}}=0.015 \mathrm{~m}^{2}$ (per injector). It is given as:

$$
Z_{x, \mathrm{u}}=\rho_{\mathrm{p}} c_{\mathrm{p}} \frac{\left(1+M_{\mathrm{p}}\right) \exp \left(\mathrm{i} \frac{k L_{\mathrm{p}}}{1-M_{\mathrm{p}}}\right)+\left(1-M_{\mathrm{p}}\right) \exp \left(-\mathrm{i} \frac{k L_{\mathrm{p}}}{1+M_{\mathrm{p}}}\right)}{\left(1+M_{\mathrm{p}}\right) \exp \left(\mathrm{i} \frac{k L_{\mathrm{p}}}{1-M_{\mathrm{p}}}\right)-\left(1-M_{\mathrm{p}}\right) \exp \left(-\mathrm{i} \frac{k L_{\mathrm{p}}}{1+M_{\mathrm{p}}}\right)}
$$

where the subscript 'p' denotes conditions in the chamber. Because $Z_{x}$ is not affected by downstream conditions, it is sufficient in the current example to only consider a domain terminating at $x=L$.

The duct transfer impedance is obtained by solving Eqs. (16) and (17) and calculating $Z=Z_{x, \text { out }}-Z_{x, \mathrm{u}}$. The results are presented in Fig. 10 for comparison. The base flow axial velocity at the inlet is adopted as the velocity scale for normalisation such that $R_{\text {in }}$ is normalised to approximately one. The frequency range of interest in the current study corresponds to $0.08<$ $k L<0.4$. The results in Fig. 10 show that, in accordance with the above proposition, a substantial increase in both the transfer resistance and transfer reactance is achieved by contracting the cross-section of the duct middle portion without altering the external dimensions of the duct. For comparison $Z_{x, \mathrm{u}}$ is also plotted in Fig. 10. As indicated above, $R_{x, \mathrm{u}}$ is almost negligible for the relevant frequency range. Meanwhile the frequency at which $X=-X_{x, \mathrm{u}}$ corresponds to the resonance frequency of the duct and upstream chamber combined.

To examine the influence of $Z_{x, \mathrm{u}}$, the duct transfer impedance was recalculated for duct (a) assuming $Z_{x, \mathrm{u}}=0$ and compared with that given in Fig. 10. The results are plotted in Fig. 11 and the influence of $Z_{x, \mathrm{u}}$ on $Z$ 
is shown to be small, as discussed above. For completeness the duct transfer impedance evaluated with Eq. (B.4) is also included in Fig. 11. Both the transfer resistance and transfer reactance are close to the exact values obtainable by solving Eqs. (16) and (17).

It is indicated in Section 3.2.1 that finiteness of the upstream chamber has negligible effect on $R_{\text {in }}$ but lowers $X_{\text {in }}$ by reducing the inlet end correction. To examine the influence of such an inlet reactance change, the transfer impedance of ducts (a) and (b) are recalculated with $Z_{x, \mathrm{u}}$ and $R_{\text {in }}$ unchanged whilst $X_{\text {in }}$ is lowered by $40 \%$ following the discussion in 3.2.1. The difference in transfer impedance between ducts (a) and (b), $\Delta Z=\Delta R+\mathrm{i} \Delta X=Z_{(\mathrm{a})}-Z_{(\mathrm{b})}$ where the subscripts denote the corresponding duct, is compared with that calculated with the transfer impedances shown in Fig. 10. The results are plotted in Fig. 12. $\Delta R$ exhibits a slight drop due to reduced non-compactness with smaller $L_{\mathrm{c}}$, but there is almost no perceivable change in $\Delta X$. The above discussion regarding the effects of duct cross-section area on the duct transfer impedance remains valid for different $Z_{\text {in }}$ of practical interests.

\subsubsection{Propagation of Acoustic Waves through Bends}

The unsteady flow between two neighbouring swirl vanes in the injector passage resembles that through a bend which is connected with straight sections both upstream and downstream. In the review given by Rostafinski [57] it is indicated that for a smooth bend of small cross-section, the evanescent higher order modes created within and in the vicinity of the bend only penetrate the straight sections for a short distance. For the frequency range and swirl vane geometry considered in the current work the lowest high order mode has decayed by more than $20 \mathrm{~dB}$ at a distance of $0.8 d_{\mathrm{v}}$ from the bend, where $d_{\mathrm{v}}$ is the pitch of swirl vane row. At the same time, the reflected waves resulting from the bend are negligible. Therefore, as far as the fundamental mode is concerned, the bend can be practically treated as a length measured along its centreline at a sufficiently low Helmholtz number. This approximation is adopted in this paper, and the pathway between two neighbouring swirl vanes is modelled as a lossless straight channel of an equivalent length but with varying cross-section area. Accordingly the flow past the front turning sections of the swirl vane row (zone II in Fig. 5) is treated as quasi one-dimensional and solved numerically from Eqs. (16)-(17) as in Section 3.2.2. The underlying geometric parameters, i.e. $A$ and $L$, as well as the base flow for this section of the injector passage, are established as described in Appendix A. It is noted that the definition of $A$ here is mostly 
geometrical because, except at the start and end points, determining the directionality of the wave front requires solving both the two-dimensional mean flow and wave equations. Nevertheless the way $A$ is defined for this section of the injector passage has negligible influence on the transfer impedance of the whole injector passage since it typically comprises only a small fraction of the total passage length.

\subsubsection{Propagation of Acoustic Waves through an Array of Flat Plates}

As depicted in Fig. 5, the rear straight sections of the swirl vane row are modelled by an infinite array of parallel flat plates of zero thickness (i.e. zone III). Applying Green's function in conjunction with the Wiener-Hopf method, Heins solved the problem of the interaction between general plane waves and such an array subject to either the Dirichlet condition [58] or the Neumann condition [59] on the plate surfaces respectively. The more specific scenario of sound propagation through blade rows with a mean flow was studied by Mani and Horvay [40] and Kaji and Okazaki [60] by different means. Mani and Horvay [40] also applied the Wiener-Hopf method, while following the dual integral equation approach. On the other hand, Kaji and Okazaki modelled each blade with a distribution of doublets, which was solved for numerically. More recently Palies et al. [38] and Candel et al. [39] revisited the same problem considering only the fundamental acoustic modes. However, to match the sudden change of acoustic propagation direction across the swirl vane trailing edges the higher order evanescent modes also need to be taken in account. The simplistic analysis in [39] does not reproduce the swirl angle dependence of acoustic propagation manifested in the exact analytical results from [40] and [59]. The current work follows the approaches of [40] and [59]. The solution given in [59] was for general wave propagation and did not take into account any base flow present in an acoustic/fluid mechanics problem, whilst in [40] only the wave amplitudes were derived but not the phase relations. In this subsection, the results from [40] and [59] are combined to obtain the complete solutions for the fundamental modes of the acoustically induced unsteady flow through the swirl vane row within the injector passage.

An illustration of the infinite array of parallel flat plates representing the rear straight sections of the swirl vane row is given in Fig. 13. The low Mach number base flow is along the positive $x$-direction. In this study the incident angle of the incident wave is equal to the swirl vane turning angle and also denoted by $\theta$, whereby $\alpha+\theta=\pi / 2$ in Fig. 13. As remarked in Section 3.1.4, 
the base flow in zones III and IV is uniform. The following linear convective wave equation can be derived from Eqs. (6)-(8):

$$
\frac{1}{c^{2}}\left(\frac{\partial}{\partial t}+\mathbf{U} \cdot \nabla\right)^{2} \tilde{\phi}-\nabla^{2} \tilde{\phi}=0
$$

At the same time, $\tilde{p}$ and $\tilde{\phi}$ are connected by the following relation [61]:

$$
\tilde{p}=-\rho\left(\frac{\partial}{\partial t}+\mathbf{U} \cdot \nabla\right) \tilde{\phi}
$$

The unsteady flow in zones III and IV combined is determined by solving Eq. (23) subject to the Neumann boundary condition on the flat plates. The general solution derived in [59] is suitable for the problem of still air. It is adapted for the current case with the following transformations:

$$
x=x_{\mathrm{t}} \sqrt{1-M^{2}}, \quad k=k_{\mathrm{t}} \sqrt{1-M^{2}}, \quad \hat{\phi}=\hat{\phi}_{\mathrm{t}} e^{\mathrm{i} k M x /\left(1-M^{2}\right)}
$$

where the variables with the subscript 't' correspond to the variables appearing in the wave equation solved in [59]. The angles in Fig. 13 are converted accordingly as:

$$
\tan \alpha_{\mathrm{t}}=\sqrt{1-M^{2}} \tan \alpha, \quad \sin \theta_{\mathrm{t}}=-\frac{\sqrt{1-M^{2}} \sin \theta}{1-M \cos \theta}
$$

By direct calculation it is found that no propagating diffracted waves exist for the geometry and frequency range considered in this paper. Meanwhile, the incident and reflected waves can be shown to travel parallel to $\xi$-axis but in opposite directions because $\alpha+\theta=\pi / 2$. Hence the solutions for the propagating waves are given as:

$$
\begin{gathered}
\hat{\phi}=C_{\phi} \exp \left(-\frac{\mathrm{i} k x}{M-1}\right)+D_{\phi} \exp \left(-\frac{\mathrm{i} k x}{M+1}\right) \quad(x \rightarrow-\infty) \\
\hat{\phi}=A_{\phi} \exp \left(-\frac{\mathrm{i} k \xi}{M \sin \alpha-1}\right)+B_{\phi} \exp \left(-\frac{\mathrm{i} k \xi}{M \sin \alpha+1}\right) \quad(\xi \rightarrow \infty)
\end{gathered}
$$

Eq. (27) describes the acoustic field between neighbouring plates upstream of the plate trailing edges (i.e. zone III in Fig. 5) and Eq. (28) is for the free space downstream of the plate array (i.e. zone IV in Fig. 5). $A_{\phi}$ and $C_{\phi}$ represent the upstream travelling waves in the corresponding regions. 
$B_{\phi}$ and $D_{\phi}$ are the downstream travelling waves. For $\hat{\phi}$ being a continuous solution downstream of the array of plates, one has from [59] the following scattering matrix between the waves:

$$
\begin{aligned}
{\left[\begin{array}{l}
B_{\phi} \\
C_{\phi}
\end{array}\right]=} & {\left[\begin{array}{cc}
G_{\mathrm{I}} & F_{\mathrm{E}} \\
F_{\mathrm{I}} & G_{\mathrm{E}}
\end{array}\right]\left[\begin{array}{l}
A_{\phi} \\
D_{\phi}
\end{array}\right] } \\
= & {\left[\begin{array}{ll}
\frac{\mathrm{i} \bar{K}_{+}^{*}\left(\sigma_{2}\right)}{k_{\mathrm{t}} \sin \left(2 \alpha_{\mathrm{t}}-\theta_{\mathrm{t}}\right)} & \frac{-b \bar{K}_{+}^{*}\left(k_{\mathrm{t}}\right)}{e^{-\mathrm{i} k_{\mathrm{t}}\left(\rho_{\mathrm{t}}-a_{\mathrm{t}}\right)}-1} \\
\frac{\mathrm{i} \sigma_{2} \bar{K}_{+}^{*}\left(\sigma_{2}\right)}{k_{\mathrm{t}} \sin \left(2 \alpha_{\mathrm{t}}-\theta_{\mathrm{t}}\right)} & \frac{-k_{\mathrm{t}} b \bar{K}_{+}^{*}\left(k_{\mathrm{t}}\right)}{e^{-\mathrm{i} k_{\mathrm{t}}\left(\rho_{\mathrm{t}}-a_{\mathrm{t}}\right)}-1}
\end{array}\right]^{-1}\left[\begin{array}{cc}
\frac{\mathrm{i} \bar{K}_{+}^{*}\left(\sigma_{1}\right)}{k_{\mathrm{t}} \sin \theta_{\mathrm{t}}} & \frac{-b \bar{K}_{+}^{*}\left(-k_{\mathrm{t}}\right)}{e^{-\mathrm{i} k_{\mathrm{t}}\left(\rho_{\mathrm{t}}+a_{\mathrm{t}}\right)}-1} \\
\frac{\mathrm{i} \sigma_{1} \bar{K}_{+}^{*}\left(\sigma_{1}\right)}{k_{\mathrm{t}} \sin \theta_{\mathrm{t}}} & \frac{k_{\mathrm{t}} b \bar{K}_{+}^{*}\left(-k_{\mathrm{t}}\right)}{e^{-\mathrm{i} k_{\mathrm{t}}\left(\rho_{\mathrm{t}}+a_{\mathrm{t}}\right)}-1}
\end{array}\right]\left[\begin{array}{l}
A_{\phi} \\
D_{\phi}
\end{array}\right] }
\end{aligned}
$$

where $\sigma_{1}$ and $\sigma_{2}$ are given as:

$$
\sigma_{1}=k_{\mathrm{t}} \cos \theta_{\mathrm{t}}, \quad \sigma_{2}=k_{\mathrm{t}} \cos \left(2 \alpha_{\mathrm{t}}-\theta_{\mathrm{t}}\right)
$$

and the split function $\bar{K}_{+}$according to $[58,59]$ is given in Appendix C including the definitions of $\rho_{\mathrm{t}}$ and $a_{\mathrm{t}}$. The symbol ' $*$ ' denotes complex conjugate.

Eq. (29) represents an unsteady flow that negotiates the sharp turn from the vane direction ( $x$-direction) to the passage axial direction ( $\xi$-direction) without loss. This is true when the base flow is absent or entering the plate array along the negative $x$-direction (i.e. corresponding to the swirl vane leading edges instead). However, in the scenario considered here the base flow leaves the plate array along the positive $x$-direction. Unsteady wakes are formed downstream of the trailing edges of plates (i.e. swirl vanes) in the presence of acoustic perturbation. A general approach to accommodate wakes in a flow solution is by introduction of discontinuities in $\hat{\phi}$ across them, which was applied by Mani and Horvay in [40]. Based on the Fourier transforms given in [40], the scattering matrix between $\left[A_{\phi} D_{\phi}\right]^{T}$ and $\left[B_{\phi} C_{\phi}\right]^{T}$ is modified as follows:

$$
\begin{aligned}
{\left[\begin{array}{l}
B_{\phi} \\
C_{\phi}
\end{array}\right] } & =\left[\begin{array}{ll}
G_{\mathrm{I}, \mathrm{w}} & F_{\mathrm{E}, \mathrm{w}} \\
F_{\mathrm{I}, \mathrm{w}} & G_{\mathrm{E}, \mathrm{w}}
\end{array}\right]\left[\begin{array}{c}
A_{\phi} \\
D_{\phi}
\end{array}\right] \\
= & {\left[\begin{array}{cc}
G_{\mathrm{I}} \cdot \frac{1+M \cos \theta_{\mathrm{t}}}{1+M \cos \left(2 \alpha_{\mathrm{t}}-\theta_{\mathrm{t}}\right)} & F_{\mathrm{E}} \cdot \frac{1-M}{1-M \cos \theta_{\mathrm{t}}} \\
F_{\mathrm{I}} \cdot \frac{1+M \cos \theta_{\mathrm{t}}}{1+M} & G_{\mathrm{E}}
\end{array}\right]\left[\begin{array}{c}
A_{\phi} \\
D_{\phi}
\end{array}\right] }
\end{aligned}
$$

where the matrix components differ from those in Eqs. (29) by factors arising due to the additional pole associated with the unsteady shed wakes. Based on the swirl vane row dimensions considered in the current study it is found 
that at a distance of $0.1 d_{\mathrm{v}}$ from the swirl vane trailing edges the magnitudes of the lowest high order evanescent modes are reduced to less than $2 \%$ of the fundamental modes in the corresponding regions. This justifies the omission of high order evanescent modes from the calculation of acoustic propagation.

The reflection and transmission coefficients for the incident wave $A_{\phi}$ are defined respectively as:

$$
R_{A}=\left|B_{\phi} / A_{\phi}\right|, \quad T_{A}=\left|\left(C_{\phi} \sqrt{\cos \theta}\right) / A_{\phi}\right|
$$

and the corresponding coefficients for $D_{\phi}$ are:

$$
R_{D}=\left|C_{\phi} / D_{\phi}\right|, \quad T_{D}=\left|B_{\phi} /\left(D_{\phi} \sqrt{\cos \theta}\right)\right|
$$

By inspection of Eq. (31) and the split function $\bar{K}_{+}$it can be seen that the reflection and transmission coefficients are functions of the incidence angle (or equivalently the swirl vane turning angle), $\theta$, Mach number, $M$, as well as the Helmholtz number, $H e_{(d)}=k d_{\mathrm{v}} . R_{A}, T_{A}, R_{D}$ and $T_{D}$ are plotted in Fig. 14 against $H e_{(d)}$ for the Mach number and swirl vane turning angle considered in the current study. Additionally, the loss coefficients of acoustic energy, calculated as $\Delta E_{A}=1-R_{A}^{2}-T_{A}^{2}$ and $\Delta E_{D}=1-R_{D}^{2}-T_{D}^{2}$ for $A_{\phi}$ and $D_{\phi}$ respectively, are also included. The influence of the Helmholtz number on the above acoustic coefficients is found to be very small since the wavelengths of the acoustic waves considered here are much larger than the pitch (i.e. $d_{\mathrm{v}}$ ) of plate array. The conversion of part of the acoustic energy into vorticity in the unsteady shed wakes is demonstrated by the non-zero loss coefficients. It is instructive to also examine the variations of the above acoustic coefficients with respect to the base flow conditions $\theta$ and $M$. This is given in Appendix D for completeness.

The dependence between $C_{\phi}$ and $D_{\phi}$ can be specified in terms of the impedance condition, $Z_{x, \text { up }}$, at a location upstream of the trailing edges of the plates. For the simplified passage geometry in Fig. $5, Z_{x \text {,up }}=Z_{x \text {,in }}^{(3)}$ at the inlet of zone III and the following linear relation between $C_{\phi}$ and $D_{\phi}$ exists:

$$
\frac{Z_{x, \mathrm{up}}}{\rho c}=\frac{Z_{x, \mathrm{in}}^{(3)}}{\rho c}=\frac{\frac{C_{\phi}}{M-1} \exp \left(\mathrm{i} \frac{k L_{\mathrm{a}}}{M-1}\right)-\frac{D_{\phi}}{M+1} \exp \left(\mathrm{i} \frac{k L_{\mathrm{a}}}{M+1}\right)}{\frac{C_{\phi}}{M-1} \exp \left(\mathrm{i} \frac{k L_{\mathrm{a}}}{M-1}\right)+\frac{D_{\phi}}{M+1} \exp \left(\mathrm{i} \frac{k L_{\mathrm{a}}}{M+1}\right)}
$$

where $L_{\mathrm{a}}$ is the length of the straight vane section included in the array. The fundamental modes $B_{\phi}, C_{\phi}$ and $D_{\phi}$ can then be determined in terms of $A_{\phi}$ 
from Eqs. (31) and (34). It is noted that downstream of the plate array there exists a convective wave due to the unsteady shed wakes. It will be demonstrated in Section 5 that this wave does not produce any pressure fluctuation or contribute to the fluctuation of volume flux in the axial direction of the injector. Hence the convective wave is not included in the calculation of transfer impedance.

\subsubsection{Outlet Impedance}

At the passage outlet the air flow effuses into the downstream squaresectioned duct as shown in Fig. 1 through a sudden expansion. Assuming

minimum pressure recovery along the passage and duct axial direction, the following expression for $C_{\sigma}$ can be obtained from Eq. (4) together with the Borda-Carnot relation (see, e.g. [62]):

$$
\left(\frac{1}{C_{\sigma}}\right)^{2}-1=\left(\frac{A_{\mathrm{d}}}{A_{\text {out }}}-1\right)^{2}
$$

where the passage outlet area, $A_{\text {out }}$, is equal to $A_{\text {in }}$ in the current study and $A_{\mathrm{d}}$ is equal to the test duct cross-section area. The outlet resistance of the injector passage, $R_{\mathrm{s}, \text { out }}$, is then calculated by Eq. (5). It is negative and offsets approximately $15 \%$ of $R_{\mathrm{s} \text {,in }}$ which is due to contraction at the passage inlet. The outlet reactance is associated with an end correction, $L_{\mathrm{c}, \text { out }}$. As the flow speed in the downstream test duct is very low $(<0.02 \mathrm{Ma})$, it is estimated using Eq. 14 with $a$ being the side of square duct. Due to the confinement of the downstream duct, $L_{\mathrm{c}, \text { out }}$ is found to be approximately $32 \%$ of the end correction corresponding to $X_{\text {rad }}$ calculated in Section 3.2.1 for the passage inlet. Combining the above, the outlet impedance of the passage is given as:

$$
Z_{\text {out }}=R_{\text {out }}+\mathrm{i} X_{\text {out }}=R_{\text {s,out }}+\mathrm{i}\left(\rho \omega L_{\text {c,out }} / A_{\text {out }}\right)
$$

\subsection{Assembly of Wave Propagation for Complete Injector Passage}

The complete solution of the unsteady passage flow associated with acoustic fluctuation is constructed by combining the results from Section 3.2. For the experimental setup described in Section 2 the upstream impedance condition is $Z_{x, \mathrm{u}}=0$. The acoustic impedance at the inlet of zone $\mathrm{I}$ is therefore $Z_{x, \text { in }}^{(1)}=Z_{\text {in }}$, where $Z_{\text {in }}$ is estimated in Section 3.2.1. Although the current calculation is based on the experimental setup, both $Z_{x, \mathrm{u}}$ and $Z_{\text {in }}$ can be substituted by the ones that match the engine condition if the combustor 
design is given. The acoustic impedance at the end of zone I, $Z_{x \text {,end }}^{(1)}$, is calculated by solving Eqs. (16) and (17) with $A=A_{\text {in }}$. Across the interface between zones I and II a jump in transfer resistance is added to account for the aforementioned concentrated hydrodynamic loss. This is given as follows by combining Eqs. (2), (4) and (5):

$$
\Delta R_{\mathrm{LE}}=\frac{\dot{m}\left(1-\eta_{\mathrm{a}}^{2}\right)}{A_{\mathrm{in}}^{2} \cos ^{2} \theta}
$$

The acoustic impedance at the inlet of zone II is then given as $Z_{x, \text { in }}^{(2)}=Z_{x, \text { end }}^{(1)}+$ $\Delta R_{\mathrm{LE}}$. Thereupon the acoustic impedance at the end of zone II, $Z_{x \text {,end }}^{(2)}$, is determined by solving Eqs. (16) and (17) with $A$ defined as in Appendix A. The flow is continuous at the interface between zones II and III. The acoustic impedance at the inlet of zone III is $Z_{x \text {,in }}^{(3)}=Z_{x \text {,end }}^{(2)}$. When the incident wave, $A_{\phi}$, is specified, the fundamental modes $B_{\phi}, C_{\phi}$ and $D_{\phi}$ in zones III and IV are solved for in terms of $A_{\phi}$ using Eqs. (31) and (34). The acoustic impedance at the end of zone IV (passage exit) is then obtained as:

$$
\begin{gathered}
Z_{x, \text { end }}^{(4)}=\rho c \frac{(1+M \cos \theta) \tilde{A}_{\phi, 4}+(1-M \cos \theta) \tilde{B}_{\phi, 4}}{(1+M \cos \theta) \tilde{A}_{\phi, 4}-(1-M \cos \theta) \tilde{B}_{\phi, 4}} \\
\tilde{A}_{\phi, 4}=A_{\phi} \exp \left(-\frac{\mathrm{i} k L_{4}}{M \sin \alpha-1}\right), \quad \tilde{B}_{\phi, 4}=B_{\phi} \exp \left(-\frac{\mathrm{i} k L_{4}}{M \sin \alpha+1}\right)
\end{gathered}
$$

where $L_{4}$ is the length of zone IV. It is noted that for the calculation of $Z_{\text {end }}^{(4)}$ the actual value of $A_{\phi}$ is not important and can be specified to any convenient value. The outlet impedance of the injector passage is estimated as outlined in Section 3.2.5. Since $Z_{x, \mathrm{u}}=0$ in the current setup, the transfer impedance of injector passage is determined as $Z=Z_{x \text {,end }}^{(4)}+Z_{\text {out }}-Z_{x, \mathrm{u}}=Z_{x \text {,end }}^{(4)}+Z_{\text {out }}$. Given the test conditions and passage geometric information, the only parameter required in the whole calculation is $\eta_{\mathrm{a}}$.

The passage transfer impedance of calculated as above is plotted in Fig. 15 together with those obtained from experiments and CFD simulations, showing all three data sets in good agreement. The transfer impedance at the quasi-steady condition (i.e. $S t \rightarrow 0$ ) is resistive only and composed of three parts. The first is associated with the flow acceleration through the injector passage and the subsequent dump loss when the flow exits the injector passage. It is equal to $R_{\mathrm{s}, \text { in }}+\left(\dot{m} / A_{\text {out }}^{2}\right)-\left(\dot{m} / A_{\text {in }}^{2}\right)+R_{\text {out }}$, which is 
essentially $\left(\dot{m} / A_{\text {out }}^{2}\right)+R_{\text {out }}$ here, and accounts for $37 \%$ of the quasi-steady state transfer impedance, $Z_{\mathrm{ss}}$, for the injector passage considered in this paper. Secondly, the contribution from the flow loss within the passage is given by Eq. (37) and constitutes $42 \%$ of $Z_{\mathrm{ss}}$. The remaining part $(21 \%)$ of $Z_{\mathrm{ss}}$ is due to unsteady vortex shedding at the swirl vane trailing edges. As shown in Fig. 14, the analytically estimated acoustic transmission and reflection coefficients remain essentially constant in the frequency range considered here, implying that the conversion of acoustic energy into unsteady shed wakes at the swirl vanes trailing edges is largely independent of the frequency of acoustic perturbation. This suggests that the rise of transfer resistance with frequency, as predicted by the above analytical approach, is mainly due to the effects of compressibility and acoustical non-compactness discussed in Section 3.2.2. Such effects are only notable at high frequencies, for example, at $S t=0.95$ they contribute $21 \%$ of the total transfer resistance of the injector passage studied here. The transfer resistance due to flow acceleration and dump loss is mainly controlled by $A_{\text {out }}$ which is generally subject to the requirement of delivering the nominal air flow rate without causing excessive pressure drop across the injector passage. Meanwhile, the transfer resistance associated with unsteady vortex shedding at the swirl vane trailing edges is determined by $\theta$ which will be mainly designed for flame stabilisation. On the other hand, the transfer resistance due to aerodynamic loss within the injector passage occurs mainly across the swirl vane row and is expected to be reduced substantially with an improved aerodynamic design. As the passage cross-section area is mostly constant, one can obtain from the transfer reactance an effective length, $L_{\text {eff }}=\frac{A_{\text {in }}}{\rho} \cdot \frac{\mathrm{d} X}{\mathrm{~d} \omega}$, for the injector passage. From the analytical estimation it is found to be approximately $1.46 L$ where $L$ is the passage length measured along the injector axis. The end corrections at the inlet and outlet amount to $0.3 \mathrm{~L}$ and $0.1 \mathrm{~L}$ respectively. Between the swirl vanes the acoustic waves travel at an angle inclined to the injector axis. This extends the acoustic path within the injector passage and contributes to the remaining difference between $L_{\text {eff }}$ and $L$. It is indicated in Section 3.2.1 that the inlet end correction of the same injector passage in a gas turbine environment is expected to be lower than that in the current experimental setup. However, following the estimation in Section 3.2.1 this difference in $L_{\mathrm{c}}$ is less than $10 \%$ of $L_{\text {eff }}$ and does not affects the current analytical study on the acoustically induced unsteady flow within the injector passage.

In a preliminary design stage the aerodynamic loss within the injector passage is usually estimated, for example, by empirical correlations. This 
is the main source of uncertainty in the estimation of $\eta_{\mathrm{a}}$ which is the single external parameter required for the transfer impedance calculation in the current section. This uncertainty is expected to decrease with the aerodynamic loss. In view of the dependence of transfer resistance on $\eta_{\mathrm{a}}$, the prediction of transfer resistance as described above is in general as accurate as the estimation of base flow aerodynamic loss. On the other hand, the transfer reactance is mainly determined by the passage geometry and the influence of base flow is $O\left(M^{2}\right)$. The prediction of transfer reactance is therefore insensitive to $\eta_{\mathrm{a}}$. Because the aerodynamic efficiencies of all the preliminary passage designs will be estimated using a common method, the modelling approach presented in the current study for the acoustically perturbed unsteady flow through the injector passage can be applied in an early design stage to provide representative quantitative comparisons for the transfer impedance of different passage designs.

\section{Effects of Swirl Vane Row Design Parameters}

Following its validation with the experimental and CFD data, the analytical approach described above to estimate the passage transfer impedance is applied to examine the influences of certain general parameters of the swirl vane row (specifically, pitch and chord length) on the overall transfer impedance of injector passage. In an actual design process, an appropriate pitch-to-chord ratio may be maintained to achieve the desired turning of the passage flow. Such a design constraint only entails setting up the corresponding simplified geometry. Nonetheless, in the current analysis the dependency between pitch and chord length has been disregarded in order to examine the effects of these two parameters independently. The current section is concerned with the swirl vane row design only and the cross-section of injector passage remains the same as the baseline configuration.

First the swirl vane row pitch is doubled whilst other dimensions remain unchanged, equivalent to reducing the vane count by half. The influence of chord length is assessed by extending the rear straight section of the swirl vane such that the total vane length measured along the injector axis, $L_{\mathrm{v}}$, is doubled, while maintaining the original vane count. The transfer impedances for these two geometric variations are estimated with the analytical approach illustrated above assuming $\eta_{\mathrm{a}}$ is unchanged. The results are presented in Fig. 16 in comparison with the baseline case. In addition, the trends for the variation of transfer impedance with pitch and chord length have also been 
examined at a fixed frequency by changing one of the two design parameters over a range of values with the other kept at the baseline value. The results for $S t=0.76$ are plotted in Fig. 17, where the letter ' $\mathrm{B}$ ' in the subscript denotes baseline values. Note that in these calculations the chord length is modified by shortening or extending the rear straight sections of swirl vanes only.

It can be seen that extending the rear section of swirl vane tends to increase both components of the transfer impedance, more notably the transfer reactance, with the physical overall length of the passage unchanged. The effects of lengthening the swirl vane by the aforementioned manner are threefold. Firstly between the rear sections of two neighbouring vanes, i.e. zone III in Fig. 5, the acoustic waves travel in a direction with an inclined angle to the injector axis and thereby over an effective length longer than the axial length. Moreover, in zone III the base flow is of the highest velocity and the effective passage sectional area normal to the acoustic propagation directions ( $A_{\text {in }} \cos \theta$ instead of $\left.A_{\text {in }}\right)$ is the smallest within the whole passage. According to the discussion in Section 3.2.2 these three factors are all expected to result in higher transfer impedance. On the other hand, a larger vane pitch is shown to have negligible effect on the transfer resistance but lead to a slight drop in the transfer reactance. It has been shown in Fig. 14 that for the frequency range of interest, the interaction between the acoustic waves and the swirl vane row, i.e. transmission and reflection, is mostly invariant with regard to the change in pitch considered here. Hence the transfer impedance is mainly determined by the apparent effective length observed by the travelling waves. With the mean centre streamline for zone II defined as in Appendix A, the combined effective length for zones II and III is reduced for a larger pitch. Therefore a gradual decrease in transfer reactance is predicted.

The swirl vane turning angle is another important design parameter for the swirl vane row. In general it is decided mainly by the requirements on air-fuel mixing and flame stabilisation. Nonetheless, it is instructive to also examine the variation of transfer impedance with swirl vane turning angle. With all the other geometric parameters fixed including the passage crosssection area, the passage transfer impedance was estimated for three different swirl vane turning angles, namely $0.8 \theta_{\mathrm{B}}, \theta_{\mathrm{B}}$ and $1.2 \theta_{\mathrm{B}}$, where $\theta_{\mathrm{B}}$ is the baseline value. The hydrodynamic loss associated with the base flow in the passage is in general a function of swirl. Because estimation of the base flow efficiency is not within the scope of the current study, this factor is disregarded here to confine the problem to the physics described by the analytical solutions dis- 
cussed in Section 3.2. Accordingly the flow is assumed lossless (ideal) within the entire passage and correspondingly, $\Delta R_{\mathrm{LE}}=0$. The results obtained based on this assumption are presented in Fig. 18. The increase in transfer resistance with larger swirl vane turning angles corresponds to an increased amount of acoustic energy being transferred to the velocity fluctuations associated with the unsteady shed wakes (mainly the transverse/circumferential velocity component as demonstrated in Section 5). On the other hand, the change in transfer reactance caused by the variation of swirl vane turning angle is negligible. It is noted that the passage cross-section area often needs to be adjusted in a practical design based on the swirl vane turning angle in order to achieve the desired air flow rate. Although it is not included in the current analysis for isolation of the effects of $\theta$ from other geometric design parameters, such a constraint can be applied in the actual design process by adjusting the simplified geometry for the analytical calculation accordingly.

\section{Convective Wave and Unsteady Shed Wakes}

Because of the assumptions of inviscid flow and zero swirl vane thickness, the idealised steady state base flow in the simplified geometry of Fig. 5 leaves the swirl vane trailing edges uniformly. However, unsteady shed wakes are formed in the presence of acoustic perturbations due to the sudden misalignment between the acoustic wave propagation directions and the base flow direction near the swirl vane trailing edges. As a result of the unsteady shed wakes, a significant part of the acoustic energy is converted into a fluctuating circumferential velocity component. It has previously been shown (see for example $[18,37,38,39])$ that while the bulk axial velocity fluctuation is mostly of acoustic nature and propagates at the speed of sound, the circumferential velocity fluctuation is convected at the base flow velocity. This was also demonstrated in the CFD simulations of [19] by tracing the unsteady flow field along a streamline of the base flow through the injector passage.

The convective wave associated with the unsteady shed wakes is often ignored in acoustic studies (e.g. [40,61]) as it does not contribute to any pressure disturbance. However, this convective wave is an integral part of the acoustically induced unsteady flow within the injector passage, and its the characteristics is of importance from the thermoacoustic perspective because the flow swirl fluctuation induced by the convective wave can affect the combustion process downstream. Palies et al. [38] presented a phenomenological analysis assuming that the unsteady vorticity generated downstream of the 
swirl vane row results in only a single fluctuating velocity component in the circumferential direction and this fluctuating velocity is uniform in the circumferential direction. However, the vorticity field derived from the analysis in [38] implies the generation of vorticity within the flow itself, whilst in a non-reacting flow vorticity can only be produced on a solid boundary and convected by the base flow (see, e.g. [62]). In view of this difficulty, the problem is revisited here by considering a more complete functional form of the convective wave. On account of the hydrodynamic nature of the unsteady shed wakes, the oscillating flow is treated as incompressible in this section without loss of the quintessence of the problem. The schematic of unsteady flow near the swirl vane trailing edges for the incompressible scenario is reproduced in Fig. 19. The base flow is along the positive $x$-direction and of a uniform velocity $U$. The upstream condition is the velocity fluctuation $\tilde{u}_{\text {up }}=\hat{u}_{\text {up }} e^{\mathrm{i} \omega t}$ along the $x$-axis. The same general assumptions as those in Section 3.2.4, i.e. inviscidity, periodicity and compactness of the wakes, apply here.

The equation to solve for a small perturbation incompressible unsteady flow is the Laplace equation $\nabla^{2} \tilde{\phi}=0$. The periodic condition downstream of the swirl vane row is $\tilde{\phi}(\xi, \eta)=\tilde{\phi}\left(\xi, \eta+d_{\mathrm{v}}\right)$, or equivalently $\tilde{\phi}(x, y)=\tilde{\phi}(x+$ $\left.d_{\mathrm{v}} \sin \theta, y+d_{\mathrm{v}} \cos \theta\right)$ in the $x-y$ coordinates. Across the wakes the conditions of continuity in pressure and the velocity component normal to the wakes apply. Instead of a full derivation of the solution to the problem formulated above, the current discussion is mainly concerned with its functional forms for the region downstream of the swirl vane row (i.e. $\xi>0$ ). The complete solution of $\tilde{\phi}=\hat{\phi} e^{\mathrm{i} \omega t}$ is comprised of components which are continuous or discontinuous across the wakes. One obvious continuous solution is:

$$
\hat{\phi}_{\mathrm{a}}=\hat{u}_{\mathrm{up}} \xi \cos \theta+C_{\xi}
$$

where $C_{\xi}$ is a constant. This solution corresponds to the axial velocity fluctuation downstream of the swirl vane row. The associated unsteady velocity is $\hat{u}_{\xi, \mathrm{a}}=\hat{u}_{\mathrm{up}} \cos \theta$ along the $\xi$-axis. When finite pressure is required for $\xi \rightarrow \infty$, the remaining continuous solutions of $\hat{\phi}$ that satisfy the periodic condition are of the form:

$$
\hat{\phi}_{\mathrm{e}}=\left[E_{n} \sin \left(\frac{2 n \pi}{d_{\mathrm{v}}} \eta\right)+F_{n} \cos \left(\frac{2 n \pi}{d_{\mathrm{v}}} \eta\right)\right] \exp \left(-\frac{2 n \pi}{d_{\mathrm{v}}} \xi\right)
$$

which decays quickly over a short distance in the positive $\xi$ direction. Thus Eq. (39) is the only persistent continuous component in the region of $\xi>0$. 
By substituting the corresponding pole into the Fourier transform given in [40] one can prove that the unsteady shed wakes are convected at the base flow velocity. The solution representing this convective wave is of the form:

$$
\hat{\phi}_{\mathrm{w}}=\left(C_{\mathrm{w}} e^{k_{\mathrm{w}} y}+D_{\mathrm{w}} e^{-k_{\mathrm{w}} y}\right) e^{-\mathrm{i} k_{\mathrm{w}} x}, \quad k_{\mathrm{w}}=\frac{\omega}{U}
$$

where the constants $C_{\mathrm{w}}$ and $D_{\mathrm{w}}$ assume different values in different regions separated by the unsteady shed wakes downstream of the swirl vane row. Without loss of generality, the region labelled with '2' in Fig. 19 is considered. From Eq. (24) one can easily verify that $\hat{\phi}_{\mathrm{w}}$ does not contribute to the pressure fluctuation. The continuity condition for pressure across the wakes is automatically satisfied by $\hat{\phi}_{\mathrm{w}}$. Application of the continuity condition for the unsteady normal velocity across the wake together with the periodic condition leads to the following relation between $C_{\mathrm{w}}$ and $D_{\mathrm{w}}$ :

$$
\left(C_{\mathrm{w}} e^{k_{\mathrm{w}} d_{\mathrm{v}} \cos \theta}-D_{\mathrm{w}} e^{-k_{\mathrm{w}} d_{\mathrm{v}} \cos \theta}\right) e^{-\mathrm{i} k_{\mathrm{w}} d_{\mathrm{v}} \sin \theta}=C_{\mathrm{w}}-D_{\mathrm{w}}
$$

The Kutta condition at the swirl vane trailing edges is that the flow direction is parallel to the swirl vane surfaces, i.e. $\hat{u}_{y}=\frac{\partial \hat{\phi}}{\partial y}=0$ at the swirl vane trailing edges, so that the unsteady flow leaves the swirl vane trailing edges smoothly without sudden change in the flow direction. When this condition is satisfied by the sum of the two persistent modes, i.e. Eqs. (39) and (41), it is expressed as follows at $(x, y)=(0,0)$, or equivalently $(\xi, \eta)=(0,0)$ :

$$
k_{\mathrm{w}}\left(C_{\mathrm{w}}-D_{\mathrm{w}}\right)-\hat{u}_{\mathrm{up}} \sin \theta \cos \theta=0
$$

which also holds for $(x, y)=\left(d_{\mathrm{v}} \sin \theta, d_{\mathrm{v}} \cos \theta\right)$, or equivalently $(\xi, \eta)=\left(0, d_{\mathrm{v}}\right)$, due to periodicity. Accordingly, the evanescent solutions, Eq. (40), should satisfy a Neumann type of condition at the same locations, which are not considered further. It is recognised that Eqs. (42) and (43) determine the values of the constants $C_{\mathrm{w}}$ and $D_{\mathrm{w}}$ in Eq. (41) uniquely. This in turn completes the solution given by Eq. (41). The unsteady velocity components in the $\xi$ and $\eta$ directions are obtained respectively as:

$$
\begin{aligned}
\hat{u}_{\xi, \mathrm{w}}= & -k_{\mathrm{w}} C_{\mathrm{w}}(\sin \theta+\mathrm{i} \cos \theta) e^{-k_{\mathrm{w}}(\sin \theta+\mathrm{i} \cos \theta)(\xi+\mathrm{i} \eta)} \\
& +k_{\mathrm{w}} D_{\mathrm{w}}(\sin \theta-\mathrm{i} \cos \theta) e^{k_{\mathrm{w}}(\sin \theta-\mathrm{i} \cos \theta)(\xi-\mathrm{i} \eta)} \\
\hat{u}_{\eta, \mathrm{w}}= & -\mathrm{i} k_{\mathrm{w}} C_{\mathrm{w}}(\sin \theta+\mathrm{i} \cos \theta) e^{-k_{\mathrm{w}}(\sin \theta+\mathrm{i} \cos \theta)(\xi+\mathrm{i} \eta)} \\
& -\mathrm{i} k_{\mathrm{w}} D_{\mathrm{w}}(\sin \theta-\mathrm{i} \cos \theta) e^{k_{\mathrm{w}}(\sin \theta-\mathrm{i} \cos \theta)(\xi-\mathrm{i} \eta)}
\end{aligned}
$$


The amplitude profiles of both $\hat{u}_{\xi, \mathrm{w}}$ and $\hat{u}_{\eta, \mathrm{w}}$ remain unchanged while they are transported downstream by the uniform base flow. These profiles at any fixed $\xi$ location are plotted against the $\eta$ coordinate in Fig. 20 for two example frequencies, $S t=0.38$ and 0.66 . Because the $\eta$-coordinate of region 2 at a $\xi$ location is $\xi \tan \theta$ to $\xi \tan \theta+d_{\mathrm{v}}$, the horizontal axes in Fig. 20 are chosen as $\eta-\xi \tan \theta$ normalised with $d_{\mathrm{v}}$. The amplitude of $\hat{u}_{\xi, \mathrm{w}}(\xi, \eta)$ is normalised with $\left|\hat{u}_{\xi, \mathrm{a}}\right|$ and that of $\hat{u}_{\eta, \mathrm{w}}(\xi, \eta)$ with $\left|\hat{u}_{\xi, \mathrm{a}} \tan \theta\right|$. The axial velocity fluctuation $\hat{u}_{\xi, \mathrm{w}}$ due to the unsteady shed wakes is shown to be significantly smaller than the unsteady bulk axial flow $\hat{u}_{\xi, \mathrm{a}}$. Moreover, through direct integration one can show $\int_{\xi \tan \theta}^{\xi \tan \theta+d_{\mathrm{v}}} \hat{u}_{\xi, \mathrm{w}}(\xi, \eta) \mathrm{d} \eta=0$ by invoking the relation of Eq. (42). This indicates that the solution $\hat{\phi}_{\mathrm{w}}$ does not contribute to the net unsteady flux through a plane normal to the $\xi$-axis (which aligns with the passage axis). On the other hand, the transverse velocity fluctuation $\left(\hat{u}_{\eta, \mathrm{w}}\right)$ due to the compact unsteady shed wakes is more significant and mostly equal to the transverse component of the unsteady bulk flow through the swirl vane row. It corresponds to the circumferential velocity fluctuation downstream of the swirl vane row which was revealed in the CFD simulations of [19].

Following the above discussion, the persistent unsteady flow downstream of the swirl vane row is essentially determined by the sum of $\hat{\phi}_{\mathrm{a}}$ and $\hat{\phi}_{\mathrm{w}}$. In [51] the unsteady velocities were examined from the CFD solutions along a circumference at the median radius of the passage exit. The results for $S t=0.38$ and 0.66 are presented in Figs. 21 and 22 together with the unsteady velocities calculated from $\hat{\phi}_{\mathrm{a}}+\hat{\phi}_{\mathrm{w}}$ at the same axial location. The reference unsteady axial velocity for the CFD results is that averaged over the whole circumference, whereas for the analytical results it is $\hat{u}_{\xi, \mathrm{a}}$. The same normalisation as in Fig. 20 is applied in the amplitude plots, while the phases of the unsteady velocities are calculated with respect to that of the corresponding reference unsteady axial velocity. The unsteady velocities obtained from CFD simulations exhibit a much more pronounced variation around the circumference of the injector passage. The dominant factor is the significant width of the wakes, which results from the finite swirl vane trailing edge thickness and the wake growth due to viscous diffusivity and turbulence. Notwithstanding, the qualitative signatures related to the idealised shed wakes can still be retrieved. The actual unsteady flow field downstream of the swirl vane is more involved than the inviscid simplification here. The main complications include the wakes in the base flow itself and the turbulence. These are in general hydrodynamic phenomena and the conversion 
of acoustic energy into vorticity is mostly a local event taking place at the trailing edges of the swirl vanes. Therefore it is argued that the evolution of the shed wakes and the associated flow unsteadiness have only small effects on the propagation of acoustic waves within the injector passage.

In contrast to the analysis in [38], it is demonstrated above that the circumferential velocity fluctuation exhibits a notable variation in phase along the transverse/circumferential direction, whilst associated with the convective wave there also exists an unsteady axial velocity component (i.e. $\hat{u}_{\xi, \mathrm{w}}$ ). From the amplitude and phase plots in Figs. 20 to 22 one can infer that the unsteady shed wake at a swirl vane trailing edge is originated from the phase difference between the unsteady flows on either side of the swirl vane. The convective wave leads to circumferential variation of the unsteady flow (in both the axial and circumferential velocity components) downstream of the swirl vane row, which becomes more significant at high frequencies. Because of the relatively large main passage radius for a lean burn injector as mentioned in Section 2, such a periodic non-uniformity in the unsteady passage flow can result in distinctive structures in the unsteady flame response, which can be of importance to the stability of the combustion process. This, however, is outside the scope of the current study and will not be considered further here. Another consequence of the convective wave is the flow swirl fluctuation, induced by the unsteady circumferential velocity. Its influence on the combustion process and its control through axial repositioning of the swirl vane row have been discussed in [20] and [38].

\section{Conclusions}

This paper presents an analytical study for the acoustically perturbed unsteady flow inside a generic gas turbine injector passage based on inviscid theory. The solution for the unsteady flow is constructed from those for the elementary geometrical features comprising the passage. The transfer impedance of the injector passage is calculated from this solution and shows good agreement with that obtained from experimental measurements and CFD simulations. One primary objective in injector design for acoustic performance is to reduce the sensitivity of injector flow to acoustic perturbations, and this can in principle be achieved by improving the transfer impedance of individual passages. The upstream acoustic condition for the fuel injector in a gas turbine engine is usually imposed by a finite-sized chamber whose inlet is connected to the compressor exit. In this work it is shown that the influence 
of such an upstream condition on the transfer impedance of injector passage is in general small for the frequency range of relevance to thermoacoustic instability. The analytical solution for the unsteady flow demonstrates that part of the acoustic energy is dissipated through unsteady vortex shedding at the swirl vane trailing edges, which increases with the swirl vane turning angle and base flow Mach number. This contributes to a considerable part of transfer resistance on top of that associated with the viscous damping within the passage and the acoustic dissipation at the passage exit due to sudden expansion. In addition, it is shown that compressibility and the acoustical non-compactness of injector passage lead to a notable rise of transfer resistance as the frequency increases. Meanwhile, the transfer reactance is mainly a function of the length of effective propagation path for acoustic waves and the passage cross-section area.

For an injector passage design, the passage inlet/outlet areas and the swirl vane turning angle are mainly specified according to the requirements on base flow under nominal operating conditions. Meanwhile, the passage length will be subject to the geometric constraints on the injector size. Based on the findings from the current work, two design practices are proposed with the objective to attain higher transfer impedance for the injector passage under the aforementioned design specifications (i.e. the inlet/outlet areas and length of the passage as well as the swirl vane turning angle have been fixed and will not be changed during the acoustic design):

1. It is recommended to minimise the cross-section area of the part of passage between inlet and outlet. This in general implies contraction downstream of the inlet followed by (smooth) diffusion to restore the passage cross-section area to the specified value at the outlet. Since the passage outlet area is unchanged, the desired base flow rate is largely retained under the nominal operating pressure drop across the passage.

2. For an axial swirler it is recommended to maximise the length of the swirl vane section which is at an inclined angle with respect to the injector axis. To accommodate a longer swirl vane within the limit of specified passage length this can also suggest turning the air flow within the passage as early as possible. An example of more ambitious design is to over turn the air flow in the front part of the passage before restoring flow swirl to the designed level in the rear part of the passage.

The viscous loss within the passage is expected to increase as a result of the design modifications guided by the two designed practices suggested 
above. Generally this can be minimised with optimised aerodynamic designs such that the desired acoustic characteristics of injector passage can be achieved with only minor compromise on the base flow efficiency.

In [19] the axial and circumferential velocity fluctuations downstream of the swirl vane row were analysed from the results of CFD simulations. Following this study the current paper provides a functional analysis on the convective wave associated with the unsteady shed wakes downstream of the swirl vane trailing edges. It is demonstrated that the unsteady shed wakes are originated from the phase difference between the acoustically induced unsteady flows on either side of each swirl vane. The convective wave comprises fluctuating axial and circumferential velocity components which are convected with the base flow. The convective wave does not contribute to any pressure fluctuation or unsteady volume flux through the injector passage. However, the associated unsteady velocities exhibit frequency-dependent variations in the circumferential direction, which become more significant at high frequencies.

By taking into account the main geometric features of the injector passage, the analytical approach presented in this work provides a cost-effective means to assess the general acoustic characteristics of a preliminary injector passage design of the axial swirler type, for example, in terms of transfer impedance. This enables the acoustic performance of injector passage to be included during exploration of a broad design envelope. The external input required in the calculation is the coefficient of aerodynamic efficiency of injector passage, which can often be estimated empirically in an early design stage. With modern computing resources it can also be obtained from a routine steady RANS calculation efficiently. The current analytical calculation could be extended to predict the transfer impedance of a multi-passage injector, although in such a case the interaction between the unsteady flows at different passage inlets and outlets also needs to be modelled, for example, by modification of the method used in [55].

\section{Acknowledgement}

This work has been funded by a Rolls-Royce/UK Engineering and Physical Sciences Research Council (EPSRC) industrial CASE studentship which is gratefully acknowledged by the authors. Numerical simulations were performed on were performed on HPC-Midlands funded by the EPSRC, Grant $\operatorname{ref} \mathrm{EP} / \mathrm{K} 000063 / 1$. 


\section{Appendix A. Base Flow in Simplified Passage Geometry}

The external setup conditions to be specified include the total pressure $p_{0}$, and total temperature, $T_{0}$, in the plenum and the nominal pressure drop, $\Delta p$, across the injector passage. Ignoring any heat transfer, the total temperature is equal to $T_{0}$ throughout the whole passage. The parameter required for the passage base flow is $\eta_{\mathrm{a}}$. Given these parameters and the simplified passage geometry, the base flow conditions $M, p$ and $T$ can be determined. Subsequently $\rho$ and $c$ are obtained from $p$ and $T$ for an ideal gas, and $U$ is equal to $M c$.

For low Mach number, the following base flow conditions at the passage inlet are obtained assuming $C_{\sigma}=1$ :

$$
M_{\text {in }} \approx \frac{A_{\text {out }} \eta_{\mathrm{a}} \cos \theta}{A_{\text {in }}} \sqrt{\frac{2 \Delta p}{\gamma p_{0}}}, \quad p_{\text {in }} \approx p_{0}-\Delta p\left(\frac{A_{\text {out }} \eta_{\mathrm{a}} \cos \theta}{A_{\text {in }}}\right)^{2}, \quad T_{\text {in }} \approx T_{0}
$$

$\rho_{\text {in }}, c_{\text {in }}$ and $U_{\text {in }}$ are found afterwards as described above. The base flow conditions are constant in zone I, i.e. $M_{1}=M_{\text {in }}, p_{1}=p_{\text {in }}, T_{1}=T_{\text {in }}, \rho_{1}=\rho_{\text {in }}$, $c_{1}=c_{\text {in }}$ and $U_{1}=U_{\text {in }}$, where the number in subscript indicates the zone.

As discussed in Section 3.2.3 the flow through the front turning section of the swirl vane row, i.e. zone II, can be treated as quasi one-dimensional. The geometrical relation for definition of the varying cross-section area, $A$, along the mean streamwise distance, $x$, is illustrated in Figs. A.1 and A.2. The turning part of the swirl vane is modelled with a circular arc, followed by a straight section that is tangent to it. In Figs. A.1 and A.2 the origin is at the centre of the circle which the circular arc of the lower swirl vane belongs to. $l_{\mathrm{v}}$ is the length of the turning part of the swirl vane measured along the axial direction of the injector. The mean streamline (denoted by the dash-dot line) is formed by the centre points of the segments of dashed lines between the swirl vanes as shown in Fig. A.1. $A$ is given as follows:

$$
A=A_{\text {in }} \frac{a_{x}-a_{\mathrm{v}}}{d_{\mathrm{v}}}
$$

where $d_{\mathrm{v}}$ is the pitch, i.e. midspan space between adjacent vanes. It is noted that the definitions of $x$ and $A$ above are approximate and not unique.

As assumed in Section 3.1.2, the total loss within the injector passage is modelled as a concentrated hydrodynamic loss at the leading edge of the swirl vane, calculated from $\Delta p$ and $\eta_{\mathrm{a}}$ with Eq. (2). For the current case 
the change in Mach number across the swirl vane leading edges due to $\Delta p_{\text {tot }}$ is small enough to be considered negligible. Based on this argument the base flow conditions $M_{2}, p_{2}$ and $T_{2}$ in zone II are determined from basic gas dynamics as below:

$$
\begin{gathered}
\frac{M_{2} A}{\left(1+\frac{\gamma-1}{2} M_{2}^{2}\right)^{\frac{\gamma+1}{2(\gamma-1)}}}=\frac{M_{\mathrm{in}} A_{\mathrm{in}}}{\left(1+\frac{\gamma-1}{2} M_{\mathrm{in}}^{2}\right)^{\frac{\gamma+1}{2(\gamma-1)}}} \\
p_{2}=\frac{p_{2, \text { tot }}}{\left(1+\frac{\gamma-1}{2} M_{2}^{2}\right)^{\frac{\gamma}{\gamma-1}}}, \quad T_{2}=\frac{T_{\infty}}{1+\frac{\gamma-1}{2} M_{2}^{2}}
\end{gathered}
$$

$\rho_{2}, c_{2}$ and $U_{2}$ are then calculated from $M_{2}, p_{2}$ and $T_{2}$ accordingly. The base flow conditions are constant and uniform from the end of turning section of the swirl vane row up to the exit of injector passage. Therefore, $M_{3}=M_{4}=$ $M_{2 \text {,end }}, p_{3}=p_{4}=p_{2, \text { end }}, T_{3}=T_{4}=T_{2, \text { end }}, \rho_{3}=\rho_{4}=\rho_{2, \text { end }}, c_{3}=c_{4}=c_{2, \text { end }}$ and $U_{3}=U_{4}=U_{2 \text {,end. }}$. For conciseness the notation of zone number for the base flow conditions is suppressed in the main text.

\section{Appendix B. Differential Equation for Impedance in Quasi One- dimensional Flow}

Using the substitution $Z_{x}=Z+Z_{x, \mathrm{u}}$, the following first order Riccati equation for the duct impedance can be obtained from Eqs. (16) to (18):

$$
\begin{aligned}
\frac{\mathrm{d} Z}{\mathrm{~d} x}= & \dot{m} \frac{\mathrm{d}}{\mathrm{d} x}\left(\frac{1}{A^{2}}\right)+\mathrm{i} \frac{\rho \omega}{\left(1-M^{2}\right) A} \cdot\left[1-\left(\frac{Z A}{\rho c}+\frac{Z_{x, \mathrm{u}} A}{\rho c}\right)^{2}\right] \\
& -\frac{4-(\gamma+1) M^{2}}{1-M^{2}} \cdot \frac{1}{2+(\gamma-1) M^{2}} \cdot \frac{\mathrm{d} M^{2}}{\mathrm{~d} x} \cdot\left(Z+Z_{x, \mathrm{u}}\right) \\
& +\frac{\rho U}{\left(1-M^{2}\right) A} \cdot \frac{\gamma-1}{\gamma} \cdot \frac{1}{p} \cdot \frac{\mathrm{d} p}{\mathrm{~d} x} \cdot\left(\frac{Z A}{\rho c}+\frac{Z_{x, \mathrm{u}} A}{\rho c}\right)^{2}
\end{aligned}
$$

In Eq. (B.1) the first term on the RHS is related to area change, c.f. Eq. (5) when $C_{\sigma}=1$. The second term can actually be derived from the acoustic propagation within a uniform duct of infinitesimal length. The last two terms are associated with density change along the duct. Usually the base flow pressure variation within the duct is much smaller than the absolute pressure, making the last term mostly negligible compared to the other terms (for example, it is $O\left(M^{4}\right)$ relative to the first term). Eq. (B.1) becomes 
singular at $M \rightarrow 1$, corresponding to the choked condition. The differential equation for the duct transfer impedance can be obtained by substituting $Z_{x}=Z+Z_{x, \mathrm{u}}$ into Eq. (B.1). Neglecting the last term on the RHS of Eq. (B.1) and retaining the Taylor expansion of the third term up to the leading order of $M^{2}$, one arrives at the following equations for transfer resistance and transfer reactance respectively:

$$
\begin{gathered}
\frac{\mathrm{d} R}{\mathrm{~d} x}=\dot{m} \frac{\mathrm{d}}{\mathrm{d} x}\left(\frac{1}{A^{2}}\right)+\frac{2 k A\left(R+R_{x, \mathrm{u}}\right)\left(X+X_{x, \mathrm{u}}\right)}{\rho c\left(1-M^{2}\right)}-2 \frac{\mathrm{d} M^{2}}{\mathrm{~d} x}\left(R+R_{x, \mathrm{u}}\right) \quad \text { (B.2) } \\
\frac{\mathrm{d} X}{\mathrm{~d} x}=\frac{\mathrm{i} \rho \omega}{\left(1-M^{2}\right) A}\left[1-\left(\frac{R+R_{x, \mathrm{u}}}{\rho c} A\right)^{2}+\left(\frac{X+X_{x, \mathrm{u}}}{\rho c} A\right)^{2}\right]-2 \frac{\mathrm{d} M^{2}}{\mathrm{~d} x}\left(X+X_{x, \mathrm{u}}\right)
\end{gathered}
$$

The initial conditions for Eqs. (B.2) and (B.3) are $R=R_{\text {in }}$ and $X=X_{\text {in }}$ at $x=0$ respectively. The following approximate solutions can be obtained for $R$ and $X$ by solving Eqs. (20) and (21):

$$
R=\frac{\int^{L} e^{\int^{x} P_{\mathrm{r}}\left(x^{\prime}\right) \mathrm{d} x^{\prime}} Q_{\mathrm{r}}(x) \mathrm{d} x+R_{\mathrm{in}}}{e^{\int^{L} P_{\mathrm{r}}(x) \mathrm{d} x}}, X=\frac{\int^{L} e^{\int^{x} P_{\mathrm{i}}\left(x^{\prime}\right) \mathrm{d} x^{\prime}} Q_{\mathrm{i}}(x) \mathrm{d} x+X_{\mathrm{in}}}{e^{\int^{L} P_{\mathrm{i}}(x) \mathrm{d} x}}
$$

where,

$$
\begin{aligned}
P_{\mathrm{r}} & =\dot{m} \frac{\mathrm{d}}{\mathrm{d} x}\left(\frac{1}{A^{2}}\right), \quad Q_{\mathrm{r}}=2 \frac{\mathrm{d} M^{2}}{\mathrm{~d} x}-\frac{2 \omega\left(X_{x, \mathrm{u}}+X_{\mathrm{o}}\right) A}{\gamma\left(1-M^{2}\right) p} \\
P_{\mathrm{i}} & =\frac{\rho \omega}{\left(1-M^{2}\right) A}\left[1-\left(\frac{R_{\mathrm{o}} A}{\rho c}\right)^{2}+\left(\frac{X_{x, \mathrm{u}} A}{\rho c}\right)^{2}\right]-2 \frac{\mathrm{d} M^{2}}{\mathrm{~d} x}+\frac{2 \omega X_{x, \mathrm{u}} A}{\gamma\left(1-M^{2}\right) p} \\
Q_{\mathrm{i}} & =2 \frac{\mathrm{d} M^{2}}{\mathrm{~d} x}-\frac{\omega X_{\mathrm{o}} A}{\gamma\left(1-M^{2}\right) p}
\end{aligned}
$$

\section{Appendix C. Split Function $\bar{K}_{+}$for Wiener-Hopf Decomposition}

From [59], the split function $\bar{K}_{+}$in Eqs. (29) is expressed explicitly as:

$$
\begin{gathered}
\bar{K}_{+}(w)=\frac{M_{+}(w)}{\bar{L}_{+}(w) \exp [-\bar{\chi}(w)]} \\
\bar{L}_{+}(w)=\prod_{n=1}^{\infty}\left(\sqrt{1-\frac{k_{\mathrm{t}}^{2} b^{2}}{n^{2} \pi^{2}}}-\frac{\mathrm{i} w b}{n \pi}\right) e^{\mathrm{i} w b / n \pi}
\end{gathered}
$$




$$
\begin{aligned}
M_{+}(w)= & \prod_{n=1}^{\infty}\left(\Gamma_{n}+\mathrm{i} \Psi_{n}\right) \exp \left[\frac{w a_{\mathrm{t}}-k_{\mathrm{t}} \rho_{\mathrm{t}}-\mathrm{i} w b}{2 \pi n}+\mathrm{i}\left(\frac{\pi}{2}-\alpha_{\mathrm{t}}\right)\right] \\
& \cdot \prod_{n=1}^{\infty}\left(\Gamma_{n}^{\prime}-\mathrm{i} \Psi_{n}^{\prime}\right) \exp \left[\frac{k_{\mathrm{t}} \rho_{\mathrm{t}}-w a_{\mathrm{t}}-\mathrm{i} w b}{2 \pi n}-\mathrm{i}\left(\frac{\pi}{2}-\alpha_{\mathrm{t}}\right)\right]
\end{aligned}
$$

where,

$$
\begin{aligned}
& a_{\mathrm{t}}=\frac{b}{\tan \alpha_{\mathrm{t}}}, \quad \rho_{\mathrm{t}}=a_{\mathrm{t}} \cos \theta_{\mathrm{t}}+b \sin \theta_{\mathrm{t}} \\
& \Gamma_{n}=\sqrt{\sin ^{2} \alpha_{\mathrm{t}}\left(1+\frac{k_{\mathrm{t}} \rho_{\mathrm{t}}}{2 \pi n}\right)^{2}-\left(\frac{b k_{\mathrm{t}}}{2 \pi n}\right)^{2}} \\
& \Gamma_{n}^{\prime}=\sqrt{\sin ^{2} \alpha_{\mathrm{t}}\left(1-\frac{k_{\mathrm{t}} \rho_{\mathrm{t}}}{2 \pi n}\right)^{2}-\left(\frac{b k_{\mathrm{t}}}{2 \pi n}\right)^{2}} \\
& \Psi_{n}=\cos \alpha_{t}\left(1+\frac{k_{\mathrm{t}} \rho_{\mathrm{t}}}{2 \pi n}\right)-\frac{w b}{2 \pi n \sin \alpha_{\mathrm{t}}} \\
& \Psi_{n}^{\prime}=\cos \alpha_{\mathrm{t}}\left(1+\frac{k_{\mathrm{t}} \rho_{\mathrm{t}}}{2 \pi n}\right)+\frac{w b}{2 \pi n \sin \alpha_{\mathrm{t}}}
\end{aligned}
$$

The infinite products in $\bar{K}_{+}$are calculated directly with the first 20 terms, beyond which the change in the products is found to be less than $0.1 \%$. It can be shown that the factor function $\bar{\chi}(w)$ is of the following form:

$$
\bar{\chi}(w)=\chi(w)+\mathrm{i} \frac{w b}{\pi}=-\mathrm{i} \frac{w b}{\pi}\left[\left(\alpha_{\mathrm{t}}-\frac{\pi}{2}\right) \cot \alpha_{\mathrm{t}}-(\ln 2) \sin \alpha_{\mathrm{t}}\right]+\mathrm{i} \frac{w b}{\pi}
$$

where $\chi(w)$ is as specified in [58].

\section{Appendix D. Variations of Reflection and Transmission Coeffi- cients with Mach number and Incidence Angle for Array of Flat Plates}

The acoustic coefficients $R_{A}, R_{D}, T_{A}, T_{D}, \Delta E_{A}$ and $\Delta E_{D}$ for an infinite array of flat plates are plotted in Fig. D.1 against the incidence angle with $H e_{(d)}=0.15$ and the base flow Mach number kept at the operating condition considered in this work. As expected, the reflection coefficients increase with $\theta$ whilst the transmission coefficients decrease. At the same time, as the ratio between the transverse/circumferential and axial velocity fluctuations 
increases with $\theta$, one should also expect a higher proportion of acoustic energy being converted into vorticity accordingly. This is demonstrated by the plots of $\Delta E_{A}$ and $\Delta E_{D}$. Similarly, the variations of the above acoustic coefficients against $M$ are examined by fixing $H e_{(d)}=0.15$ and $\theta$ at the design value of the swirl vane row considered in the current study. The results are presented Fig. D.2. It is shown that the loss coefficients of acoustic energy increase with the base flow Mach number. This corresponds to more vorticity energy being convected downstream within a unit time due to a higher base flow velocity.

\section{References}

[1] T. C. Lieuwen, B. T. Zinn, The role of equivalence ratio oscillations in driving combustion instabilities in low $\mathrm{NO}_{\mathrm{x}}$ gas turbines, Symposium (International) on Combustion 27 (1998) 1809-1816.

[2] T. Lieuwen, Modeling premixed combustion - acoustic wave interactions: a review, Journal of Propulsion and Power 19 (2003) 765-781.

[3] S. R. Stow, A. P. Dowling, A time-domain network model for nonlinear thermoacoustic oscillations, Journal of engineering for gas turbines and power 131 (2009) 031502.

[4] Y. Huang, V. Yang, Dynamics and stability of lean-premixed swirlstabilised combustion, Progress in Energy and Combustion Science 35 (2009) 293-364.

[5] P. Palies, T. Schuller, D. Durox, L. Y. M. Gicquel, T. Poinsot, Acoustically perturbed turbulent premixed swirling flames, Physics of Fluids 23 (2010) 037101.

[6] B. G. Franzelli, E. Riber, L. Y. M. Gicquel, T. Poinsot, Large eddy simulation of combustion instabilities in a lean partially premixed swirled flame, Combustion and Flame 159 (2012) 621-637.

[7] T. Yi, D. A. Santavicca, Combustion instability and flame structure of turbulent swirl-stabilized liquid-fueled combustion, Journal of Propulsion and Power 28 (2012) 1000-1014. 
[8] N. A. Worth, J. R. Dawson, Self-excited circumferential instabilities in a model annular gas turbine combustor: Global flame dynamics, Proceedings of the Combustion Institute 34 (2013) 3127-3134.

[9] M. Schulze, T. Hummel, N. Klarmann, F. Berger, B. Schuermans, T. Sattelmayer, Linearized Euler equations for the prediction of linear high-frequency stability in gas turbine combustors, Journal of Engineering for Gas Turbines and Power 139 (2017) 031510.

[10] O. Lucca-Negro, T. O'Doherty, Vortex breakdown: a review, Progress in Energy and Combustion Science 27 (2001) 431-481.

[11] L. Graftieaux, M. Michard, N. Grosjean, Combining PIV, POD and vortex identification algorithms for the study of unsteady turbulent swirling flows, Measurement Science and Technology 12 (2001) 1422-1429.

[12] P. Jochmann, A. Sinigersky, M. Hehle, O. Schäfer, R. Koch, H.-J. Bauer, Numerical simulation of precessing vortex breakdown, International Journal of Heat and Fluid Flow 27 (2006) 192-203.

[13] S. Wang, V. Yang, G. Hsiao, S. Hsieh, H. C. Mongia, Large-eddy simulation of gas-turbine swirl injector flow dynamics, Journal of Engineering for Gas Turbines and Power 88 (2007) 143-168.

[14] A. Spencer, J. J. McGuirk, K. Midgley, Vortex breakdown in swirling fuel injector flows, ASME Journal of Engineering for Gas Turbines and Power 130 (2008) 021503-1-8.

[15] L. Cheng, M. Dianat, A. Spencer, J. J. McGuirk, Validation of LES predictions of scalar mixing in high-swirl fuel injectors, Flow Turbulence and Combustion 88 (2012) 143-168.

[16] J. Cohen, J. Hibshman, An experimental study of combustor air swirler acoustic and fluid dynamic sensitivities, in: PERC 9th Symposium on Propulsion, Cleveland, United States, 1997.

[17] S. Wang, S.-Y. Hsieh, V. Yang, Vortical dynamics and acoustic response in gas-turbine swirl-stabilised injectors, in: 40th Aerospace Sciences Meeting \& Exhibit, Reno, United States, 2002. 
[18] S. Wang, V. Yang, Unsteady flow evolution in swirl injectors with radial entry. II. External excitations, Physics of Fluids 17 (2005) 045107.

[19] J. Su, A. Garmory, J. F. Carrotte, The aerodynamic response of fuel injector passages to incident acoustic waves, in: Proceedings of ASME Turbo Expo 2015: Turbine Technical Conference and Expopsition, GT2015-43248, Montreal, Canada, 2015.

[20] N. C. W. Treleaven, J. Su, A. Garmory, G. J. Page, The response to incident acoustic waves of the flow field produced by a multi-passage leanburn aero-engine fuel injector, in: Proceedings of ASME Turbo Expo 2017: Turbomachinery Technical Conference and Expopsition, GT201764527, Charlotte, USA, 2017.

[21] B. Schuermans, V. Bellucci, F. Guethe, F. Meili, P. Flohr, C. O. Paschereit, A detailed analysis of thermoacoustic interaction mechanisms in a turbulent premixed flame, ASME Paper No. GT2004-53831 (2004).

[22] W. Meier, P. Weigand, X. R. Duan, R. Giezendanner-Thoben, Detailed characterization of the dynamics of thermoacoustic pulsations in a lean premixed swirl flame, Combustion and Flame 150 (2007) 2-26.

[23] K. T. Kim, J. G. Lee, B. D. Quay, D. A. Santavicca, Response of partially premixed flames to acoustic velocity and equivalence ratio perturbations, Combustion and Flame 157 (2010) 1731-1744.

[24] K. T. Kim, S. Hochgreb, Effects of nonuniform reactant stoichiometry on thermoacoustic instability in a lean-premixed gas turbine combustor, Combustion Science and Technology 184 (2012) 608-628.

[25] C. Huang, C. Yoon, R. Gejji, W. E. Anderson, V. Sankaran, Computational study of combustion dynamics in a single-element lean direct injection gas turbine combustor, in: 52th Aerospace Sciences Meeting, American Institute of Aeronautics and Astronautics, 2014.

[26] A. P. Dowling, S. R. Stow, Acoustic analysis of gas turbine combustors, Journal of propulsion and power 19 (2003) 751-764.

[27] C. O. Paschereit, B. Schuermans, V. Bellucci, P. Flohr, Integrated analytical, numerical and experimental design process. Chapter 15. In: 
Combustion instabilities in gas turbine engines: operational experience, fundamental mechanisms, and modeling, Progress in astronautics and aeronautics (2005).

[28] W. Polifke, C. Paschereit, Determination of thermo-acoustic transfer matrices by experiment and computational fluid dynamics, ERCOFTAC bulletin 38 (1998).

[29] B. B. Schuermans, W. Polifke, C. O. Paschereit, Modeling transfer matrices of premixed flames and comparison with experimental results, in: ASME 1999 International Gas Turbine and Aeroengine Congress and Exhibition, American Society of Mechanical Engineers, 1999, p. V002T02A024.

[30] A. Fischer, C. Hirsch, T. Sattelmayer, Comparison of multi-microphone transfer matrix measurements with acoustic network models of swirl burners, Journal of Sound and Vibration 298 (2006) 73-83.

[31] C. Yoon, O. Graham, F. Han, K. Kim, K. Maxted, T. Caley, J. G. Lee, LES-based scattering matrix method for low-order acoustic network models, in: ASME Turbo Expo 2017: Turbomachinery Technical Conference and Exposition, American Society of Mechanical Engineers, 2017.

[32] P. Cobo, M. Cuesta, M. Siguero, Comparison of models describing double layer microperforated absorbers, Noise Control Engineering Journal 57 (2009) 10-15.

[33] T. Herdtle, J. Stuart Bolton, N. N. Kim, J. H. Alexander, R. W. Gerdes, Transfer impedance of microperforated materials with tapered holes, The Journal of the Acoustical Society of America 134 (2013) 4752-4762.

[34] J. Tournadre, K. Förner, P. Martinez-Lera, W. Polifke, W. Desmet, Determination of acoustic impedance for helmholtz resonators through incompressible unsteady flow simulations, in: 22nd AIAA/CEAS Aeroacoustics Conference, 2016, p. 2917.

[35] J. M. Beér, N. A. Chigier, Combustion aerodynamics, New York (1972).

[36] A. H. Lefebvre, Gas Turbine Combustion: Alternative Fuels and Emissions, CRC press, 2010. 
[37] T. Komarek, W. Polifke, Impact of swirl fluctuations on the flame response of a perfectly premixed swirl burner, Journal of Engineering for Gas Turbines and Power 132 (2010) 061503.

[38] P. Palies, D. Durox, T. Schuller, S. Candel, The combined dynamics of swirler and turbulent premixed swirling flames, Combustion and Flame 157 (2010) 1698-1717.

[39] S. Candel, D. Durox, T. Schuller, J.-F. Bourgouin, J. P. Moeck, Dynamics of swirling flames, Annual review of fluid mechanics 46 (2014) $147-173$.

[40] R. Mani, G. Horvay, Sound transmission through blade rows, Journal of Sound and Vibration 12 (1970) 59-83.

[41] J. Kopitz, A. Huber, T. Sattelmayer, W. Polifke, Thermoacoustic stability analysis of an annular combustion chamber with acoustic low order modeling and validation against experiment, in: ASME Turbo Expo 2005: Power for Land, Sea, and Air, American Society of Mechanical Engineers, 2005, pp. 583-593.

[42] R. C. Steele, L. H. Cowell, S. M. Cannon, C. E. Smith, Passive control of combustion instability in lean premixed combustors, Journal of Engineering for Gas Turbines and Power 122 (2000) 412-419.

[43] J. Rupp, J. Carrotte, M. Macquisten, The use of perforated damping liners in aero gas turbine combustion systems, Journal of Engineering for Gas Turbines and Power 134 (2012) 071502.

[44] J. Rupp, Acoustic Absorption and the Unsteady Flow Associated with Circular Apertures in a Gas Turbine Environment, PhD Thesis, Loughborough University, 2013.

[45] OpenFOAM, www.openfoam.com v. 2.1.1 (2013).

[46] R. I. Issa, Solution of the implicitly discretised fluid flow equations by operator-splitting, Journal of Computational Physics 62 (1985) 40-65.

[47] T. Luong, M. S. Howe, R. S. McGowan, On the Rayleight conductivity of a bias-flow aperture, Journal of Fluids and Structures 21 (2005) 769778 . 
[48] V. Bellucci, P. Flohr, C. O. Paschereit, Numerical and experimental study of acoustic damping generated by perforated screens, AIAA Journal 42 (2004) 1543-1549.

[49] M. S. Howe, The damping of sound by wall turbulent shear layers, The Journal of the Acoustical Society of America 98 (1995) 1723-1730.

[50] C. Weng, S. Boij, A. Hanifi, The attenuation of sound by turbulence in internal flows, The Journal of the Acoustical Society of America 133 (2013) 3764-3776.

[51] J. Su, Unsteady Aerodynamic Response Characteristics of Gas Turbine Fuel Injectors, PhD Thesis, Loughborough University, 2017.

[52] L. E. Kinsler, A. R. Frey, A. B. Coppens, J. V. Saunders, Fundamentals of Acoustics, John Wiley \& Sons, Inc., 2000.

[53] J. W. S. Rayleigh, The Theory of Sound, Vol. 2), 2nd ed., Dover Publications Inc., New York, 1945.

[54] A. Selamet, Z. L. Ji, R. A. Kach, Wave reflections from duct terminations, Journal of the Acoustical Society of America 109 (2001) 13041311.

[55] U. Ingard, On the theory and design of acoustic resonators, The Journal of the acoustical society of America 25 (1953) 1037-1061.

[56] D. Yang, A. S. Morgans, The acoustics of short circular holes opening to confined and unconfined spaces, Journal of Sound and Vibration 393 (2017) 41-61.

[57] W. Rostafinski, Monograph on propagation of sound waves in curved ducts, NASA, 1991.

[58] A. E. Heins, The Green's function for periodic structures in diffraction theory with an application to parallel plate media, I, Journal of Mathematics and Mechanics 6 (1957) 401-426.

[59] A. E. Heins, The Green's function for periodic structures in diffraction theory with an application to parallel plate media, II, Journal of Mathematics and Mechanics 6 (1957) 629-639. 
[60] S. Kaji, T. Okazaki, Propagation of sound waves through a blade row: II analysis based on the acceleration potential method, Journal of Sound and Vibration 11 (1970) 355-375.

[61] R. M. Munt, Acoustic radiation from a circular cylinder in a subsonic stream, IMA Journal of Applied Mathematics 16 (1975) 1-10.

[62] G. K. Batchelor, An introduction to fluid dynamics, Cambridge university press, 2000. 


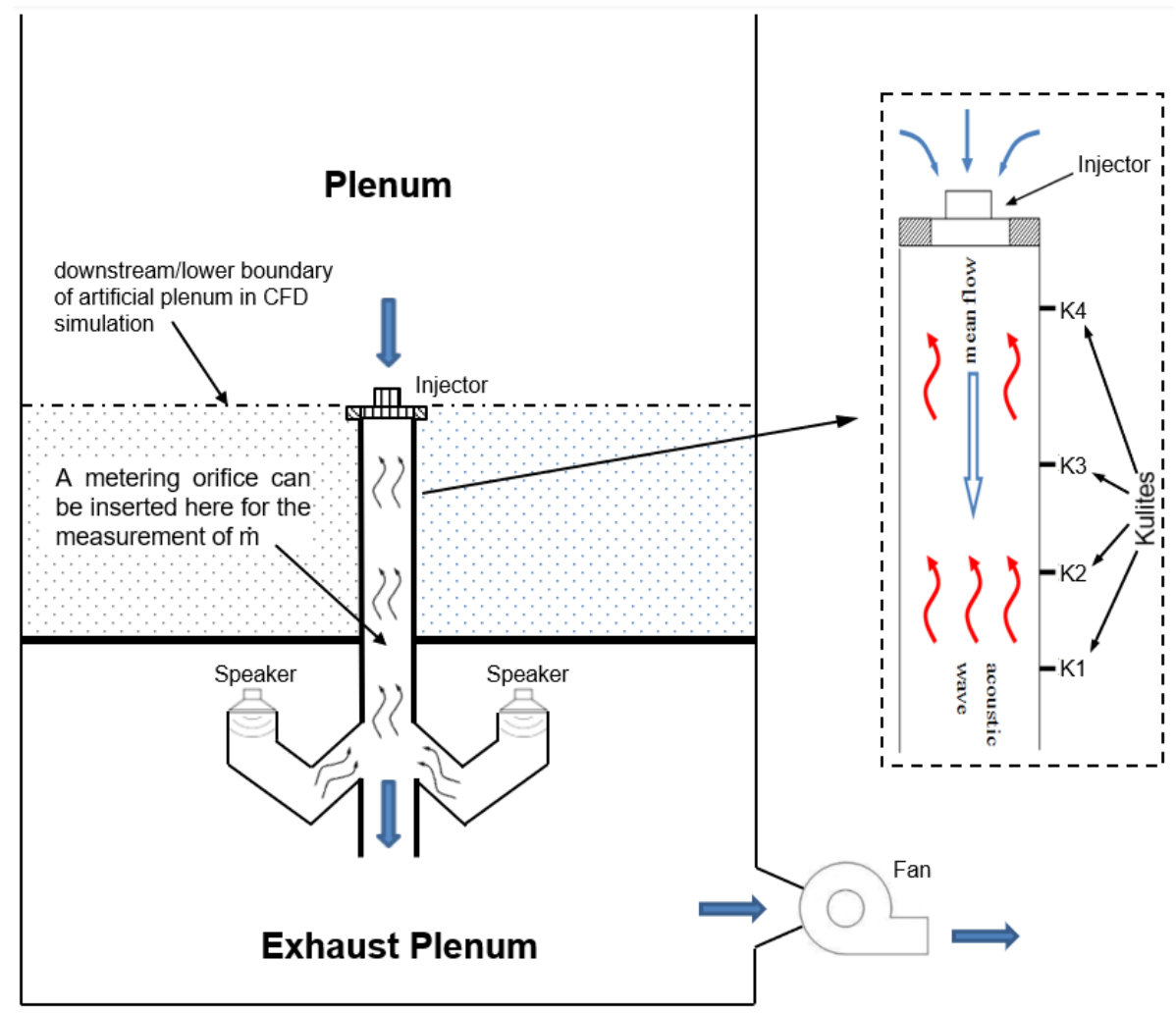

Figure 1: Schematic of experimental setup 


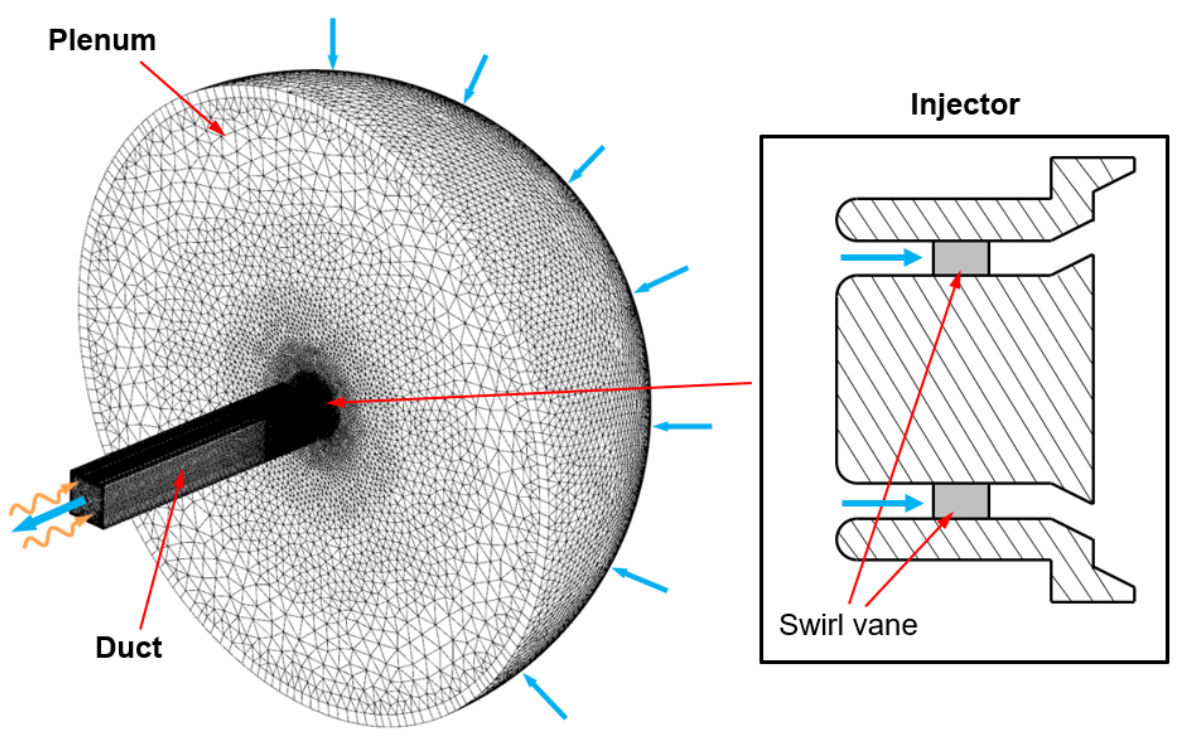

Figure 2: Computational domain and grid for CFD simulation of injectors 


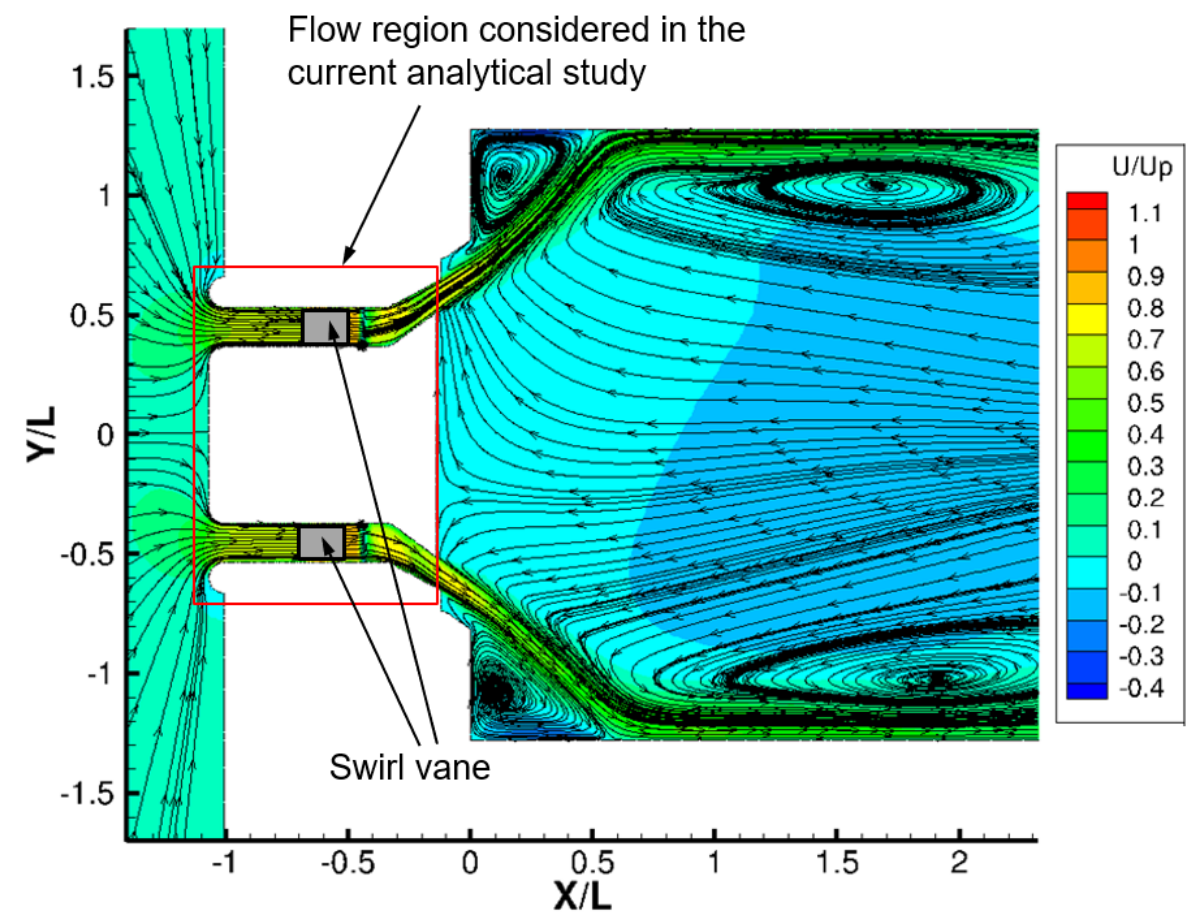

Figure 3: Flow field in centre cross-plane of single passage injector [19] 


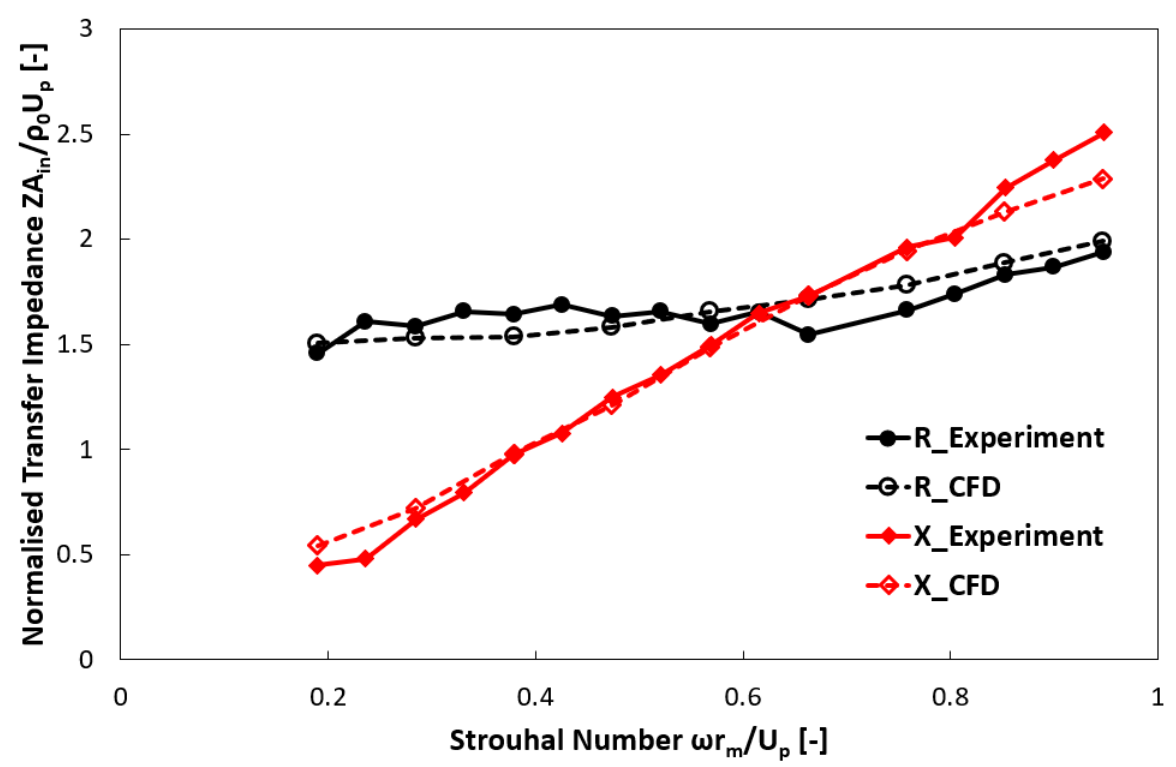

Figure 4: Transfer impedance of baseline single-passage injector configuration

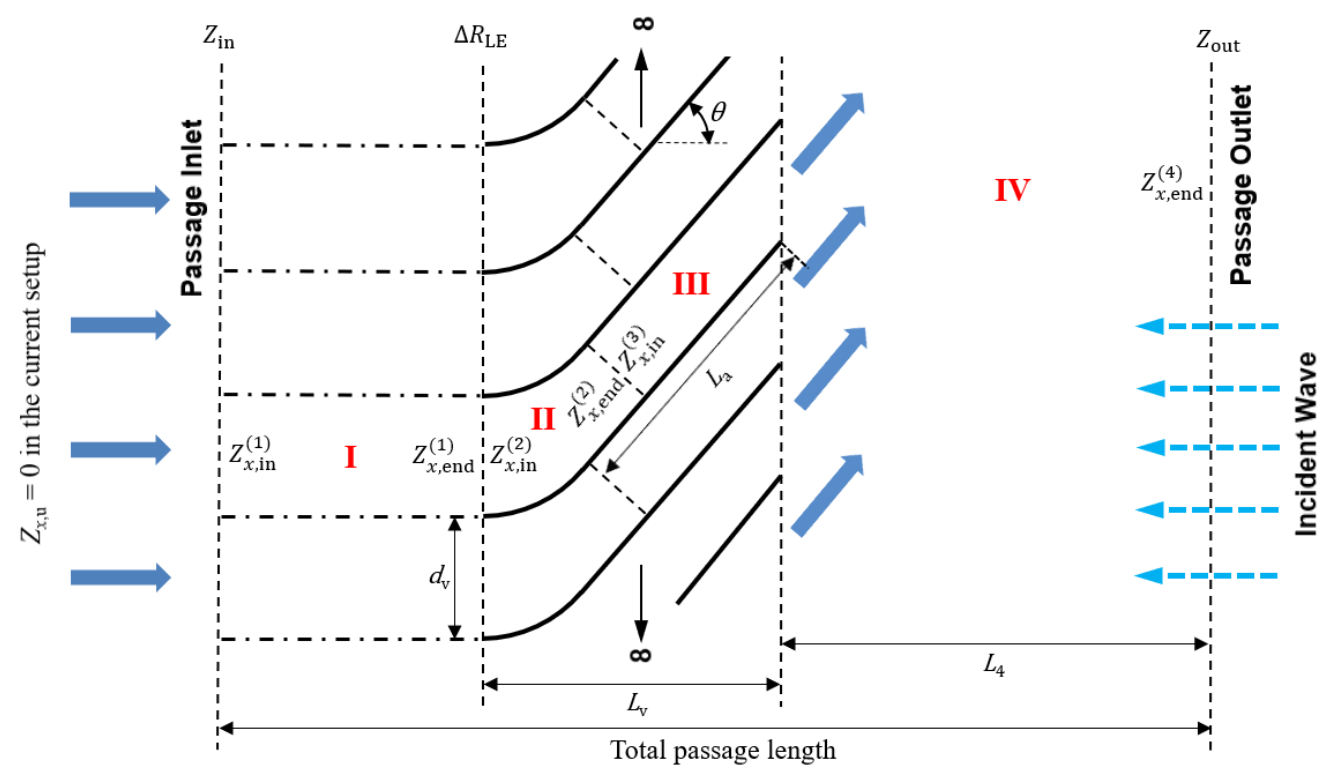

Figure 5: Simplified geometry for analytical study 


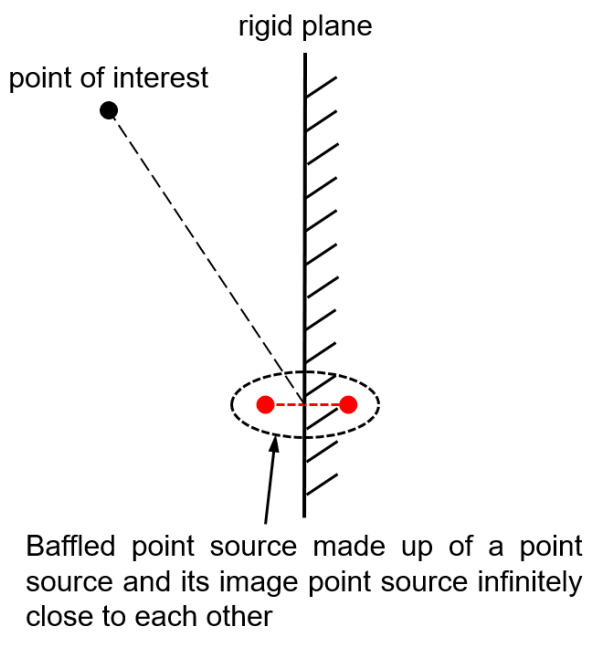

(a) Baffled point source

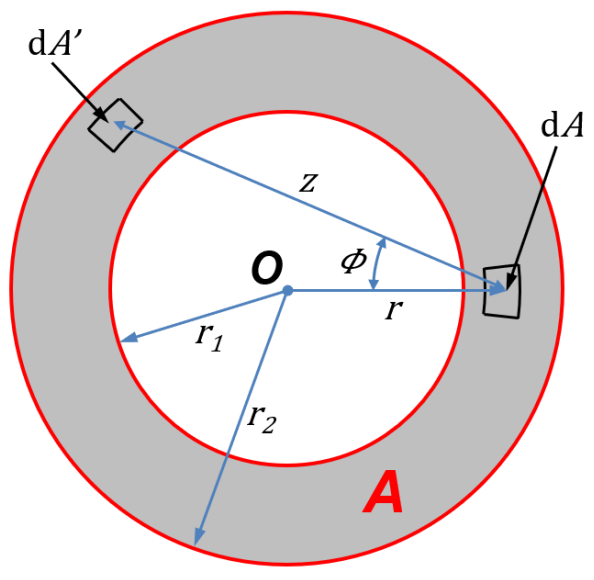

(b) Passage inlet plane

Figure 6: Inlet plane of injector passage as vibrating annular piston

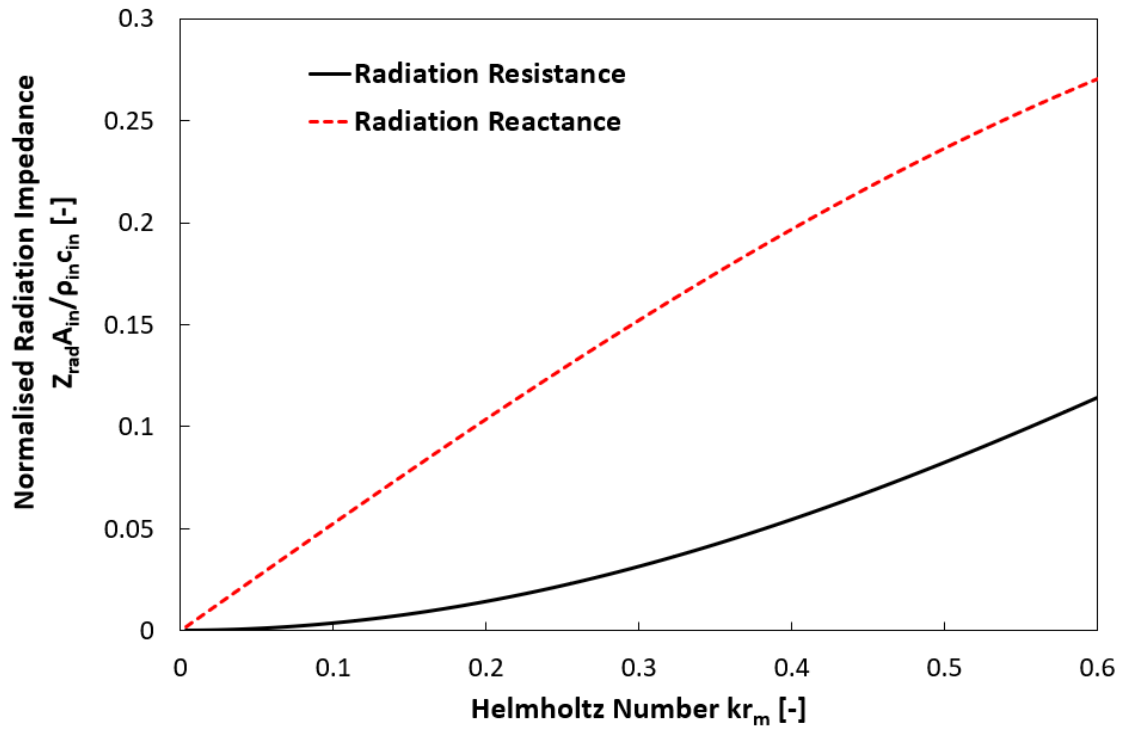

Figure 7: Radiation impedance of flanged annular inlet 


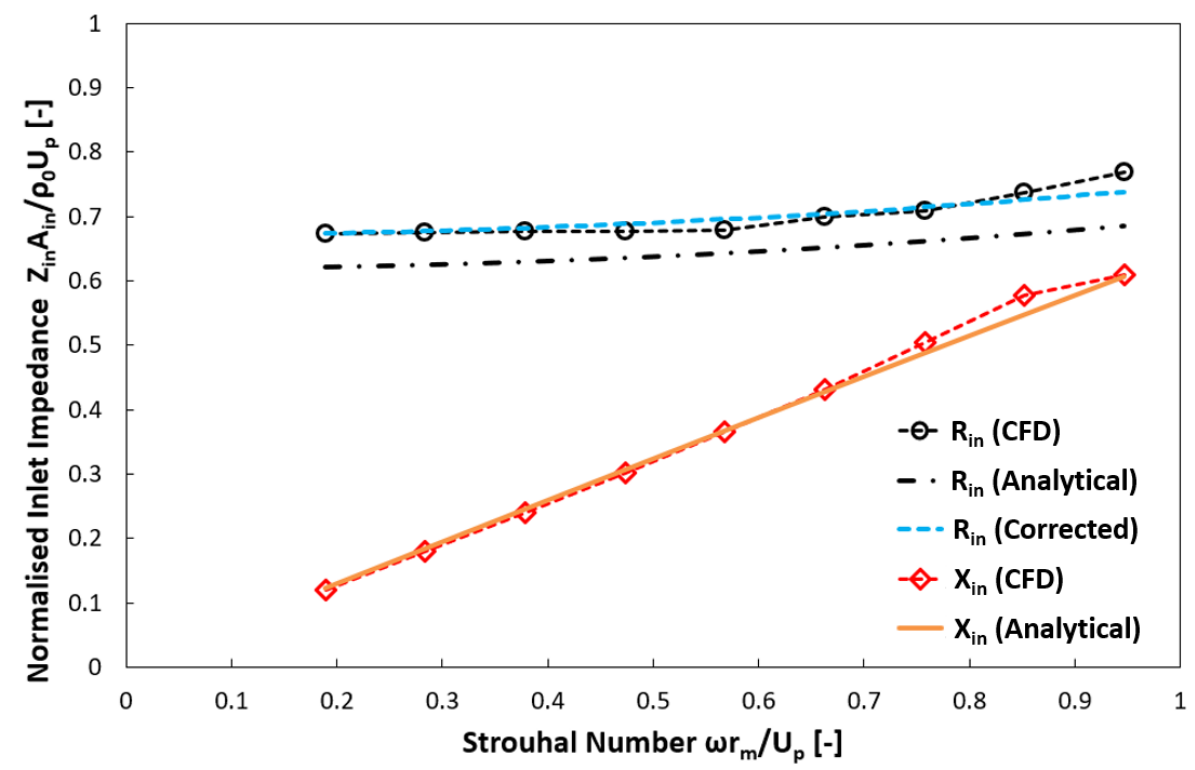

Figure 8: Baseline passage inlet impedance

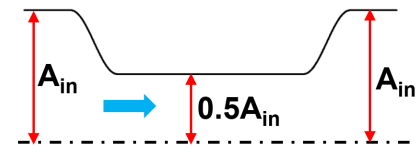

(a) Contraction

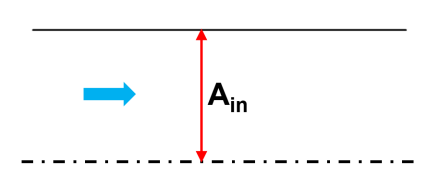

(b) Uniform

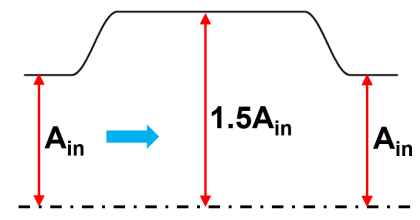

(c) Expansion

Figure 9: Straight ducts with varying cross-section areas 


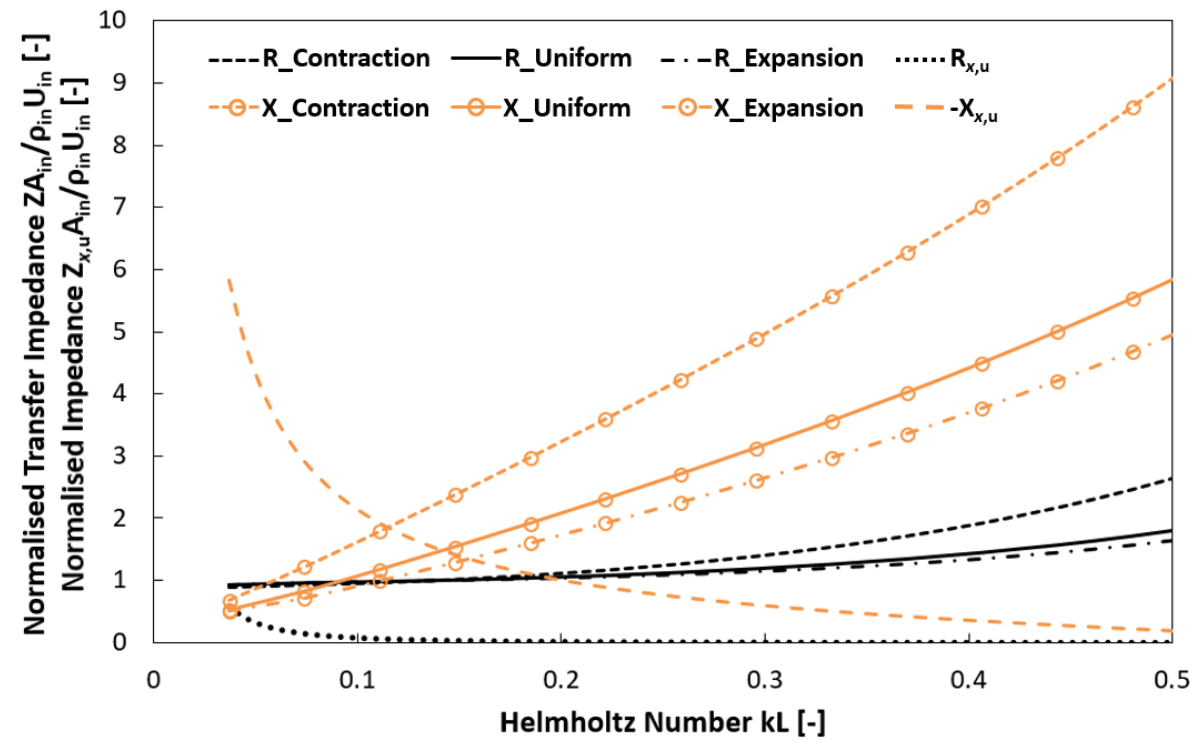

Figure 10: Transfer impedance of straight ducts with varying cross-section areas

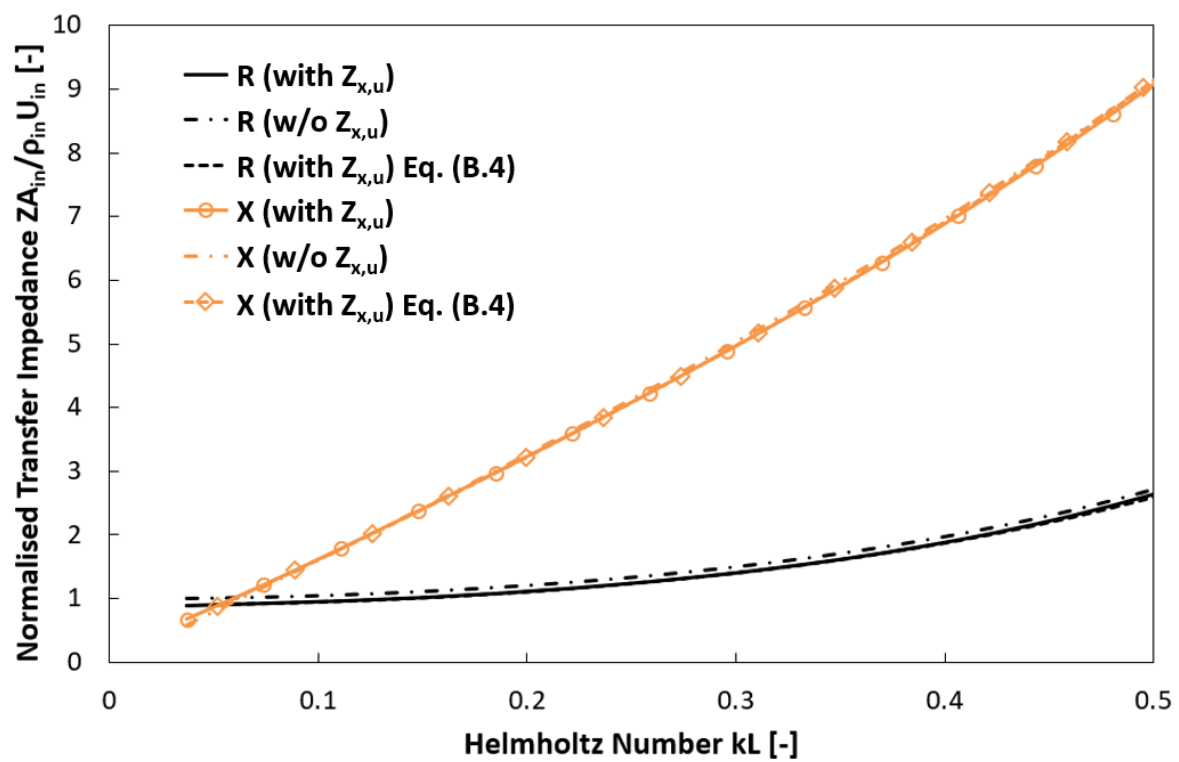

Figure 11: Influence of upstream impedance on transfer impedance of duct with contraction 


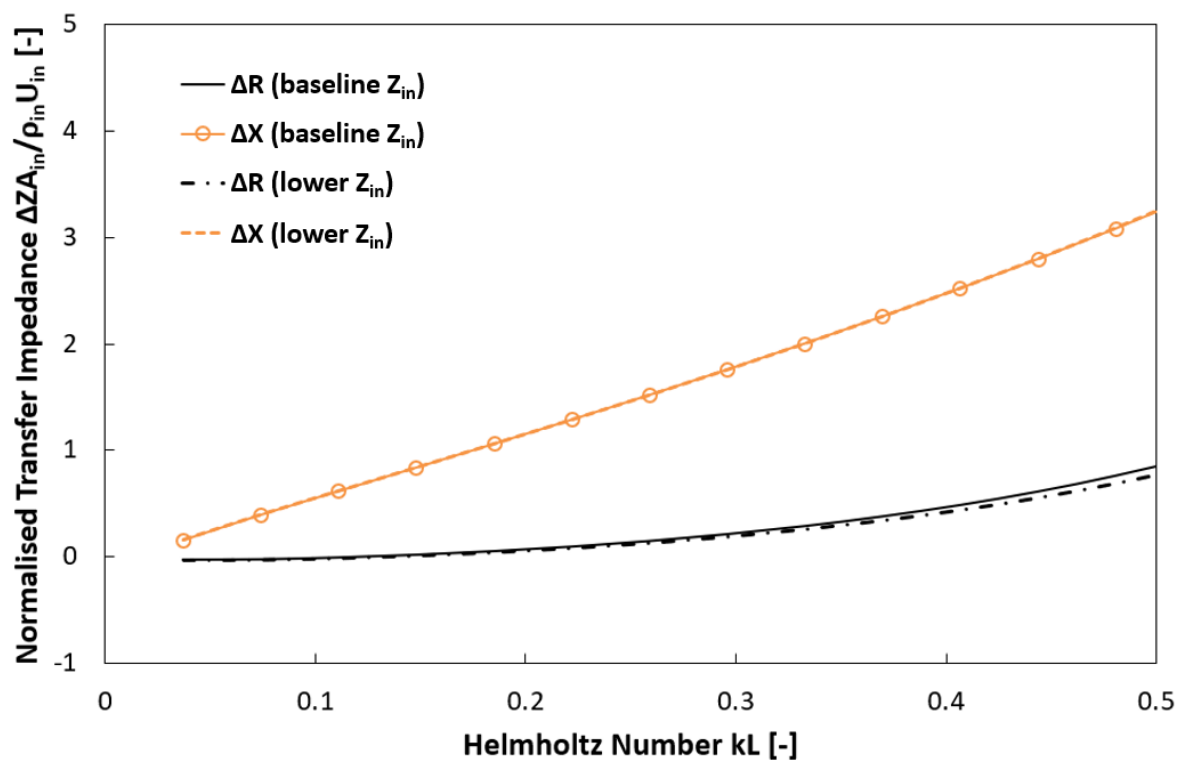

Figure 12: Influence of inlet reactance on transfer impedance change due to duct crosssection contraction

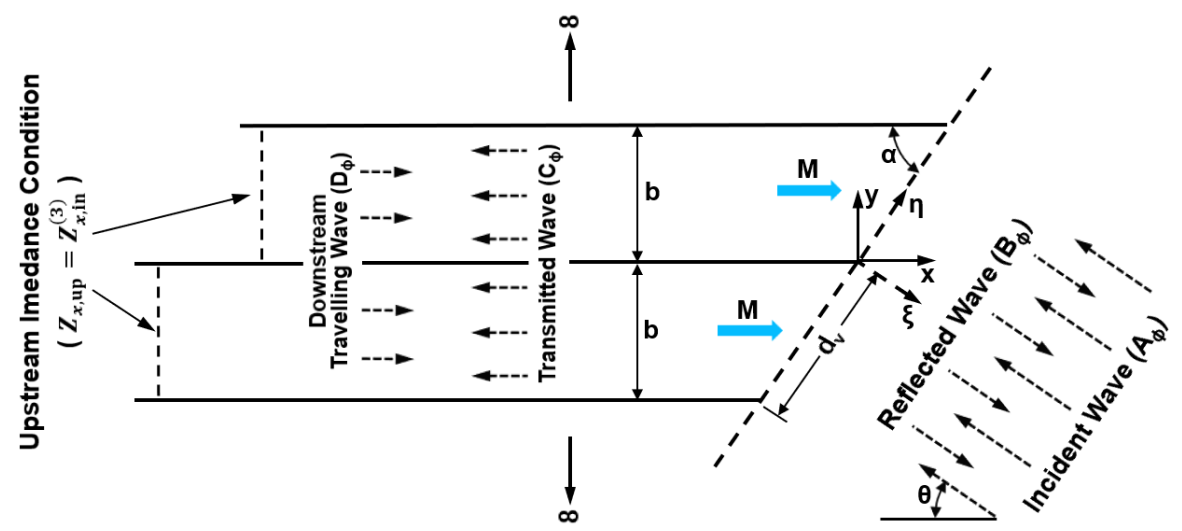

Figure 13: Acoustic wave incident upon an infinite array of parallel flat plates 


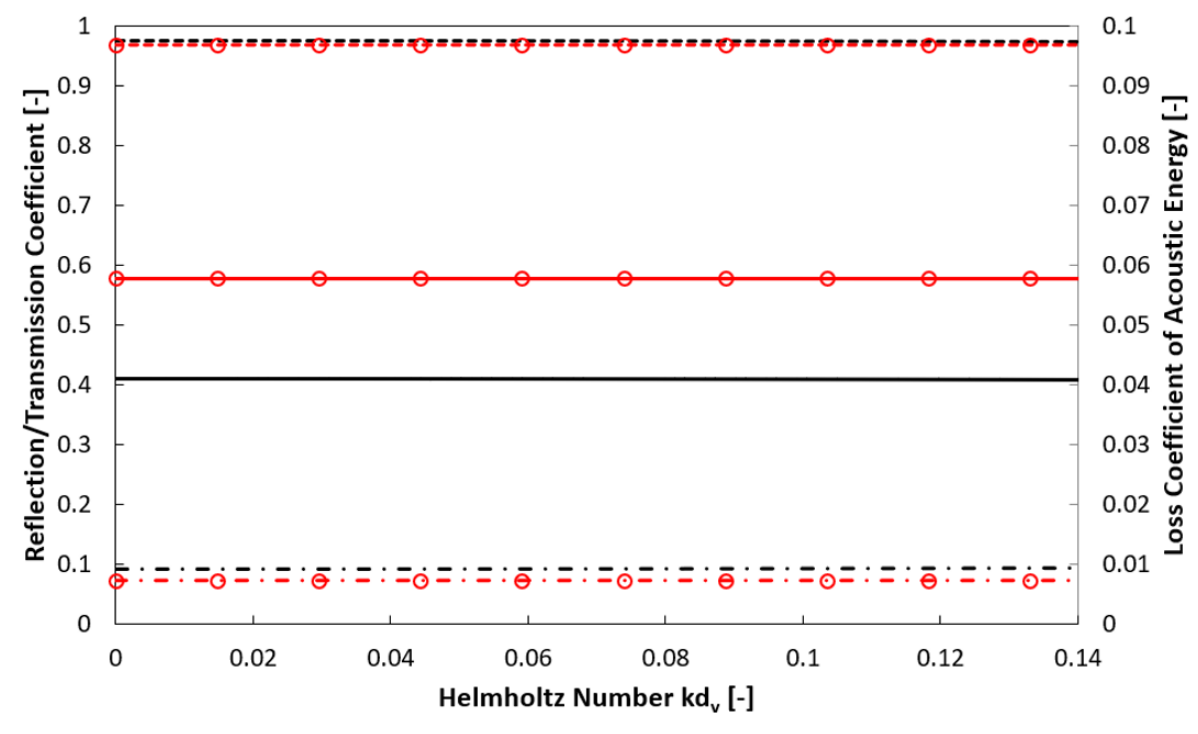

$$
-\cdots R_{A}-\cdots T_{A}-\odot \cdot R_{D}-\Theta-T_{D}-\Delta E_{A}-\Delta E_{D}
$$

Figure 14: Reflection and transmission coefficients as function of Helmholtz number

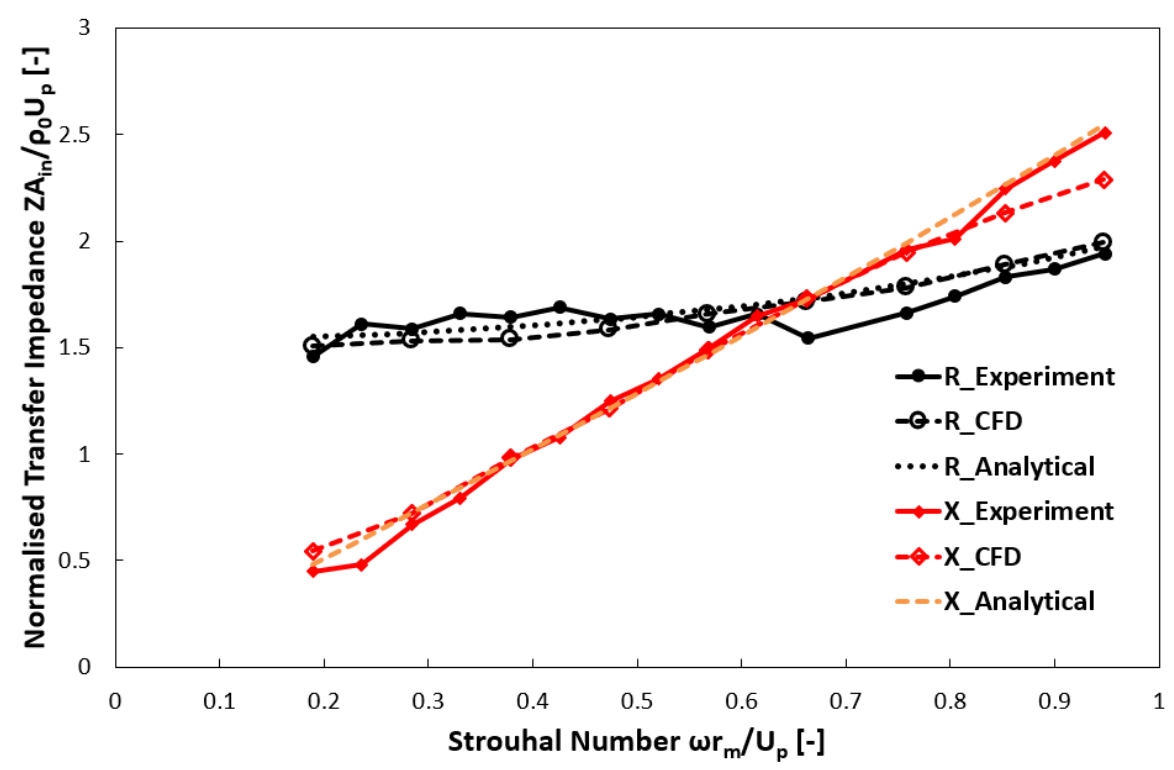

Figure 15: Analytical estimation of transfer impedance of injector passage 


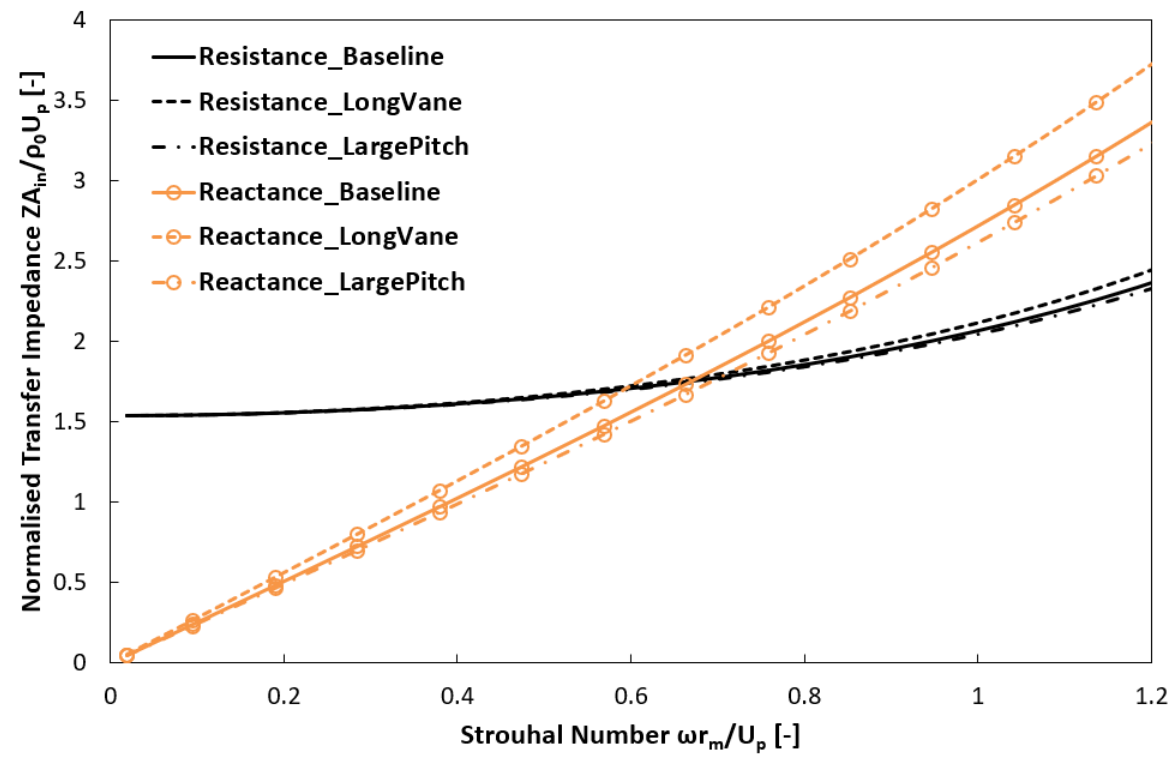

Figure 16: Transfer impedances of passages with different chord and pitch of swirl vane row

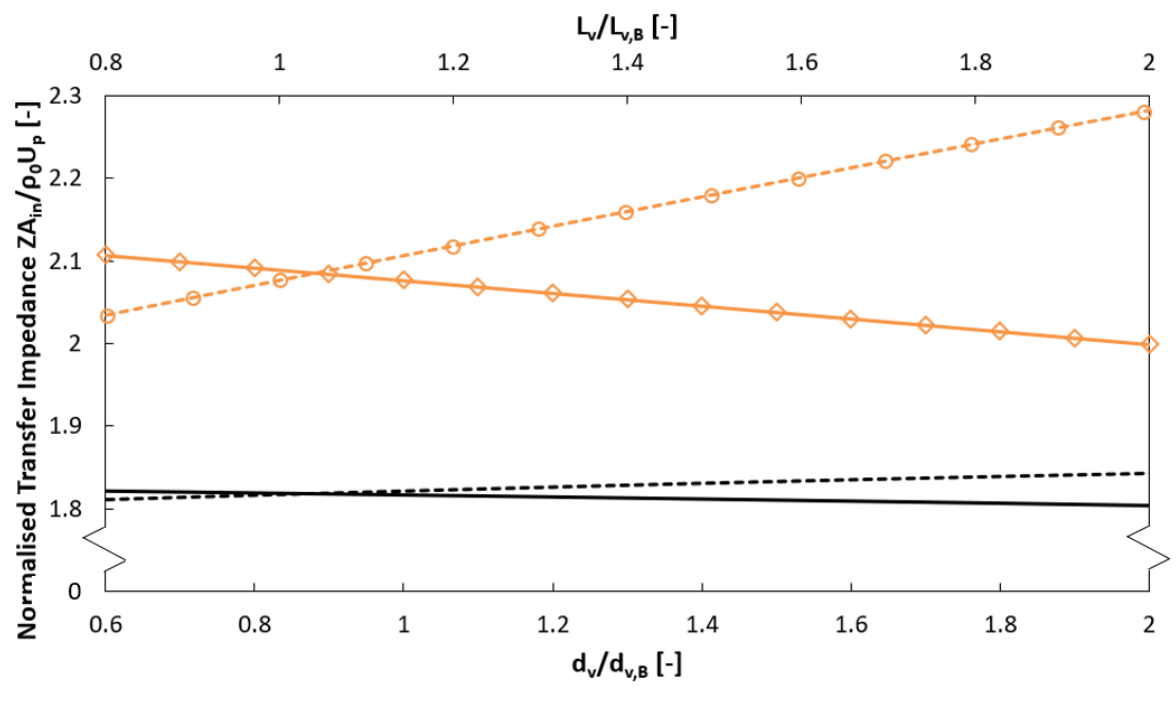

$-\mathbf{R}$ (pitch varied) $\rightarrow \mathbf{X}$ (pitch varied) --- $\mathbf{R}$ (chord varied) $-\odot-\mathbf{X}$ (chord varied)

Figure 17: Variation of transfer impedance with vane length and pitch $(S t=0.76)$ 


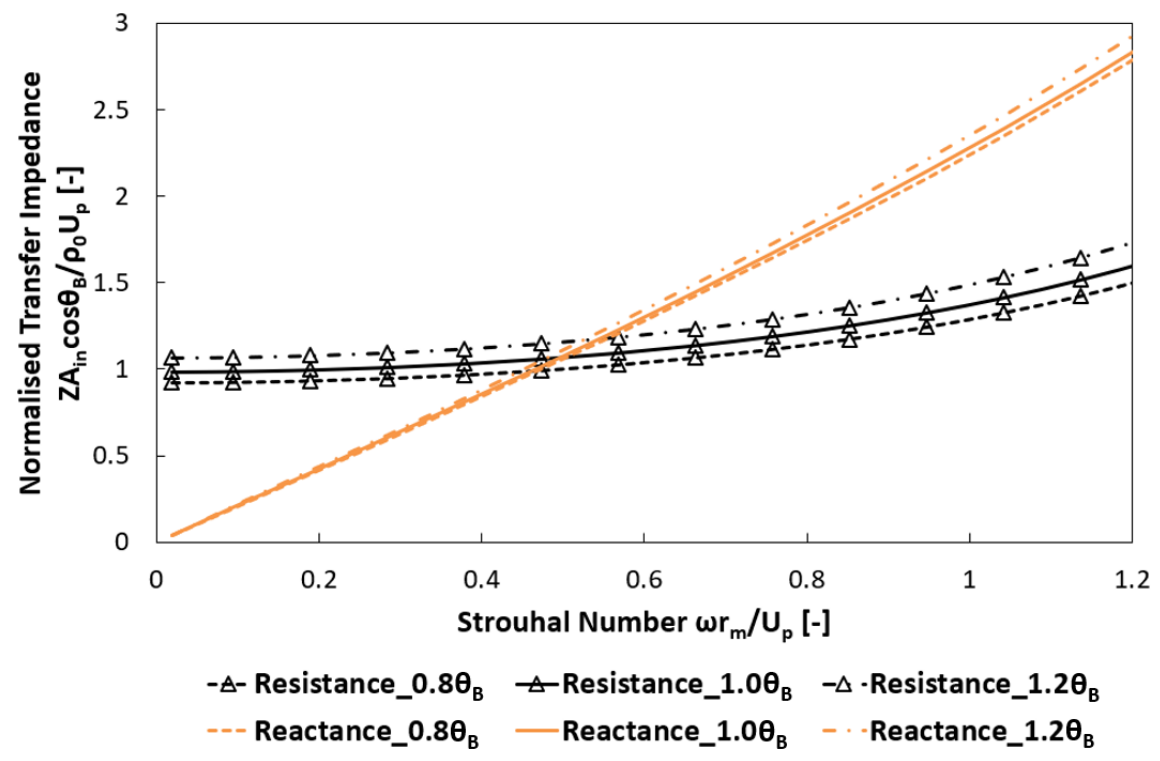

Figure 18: Variation of transfer impedance with change of swirl vane turning angle

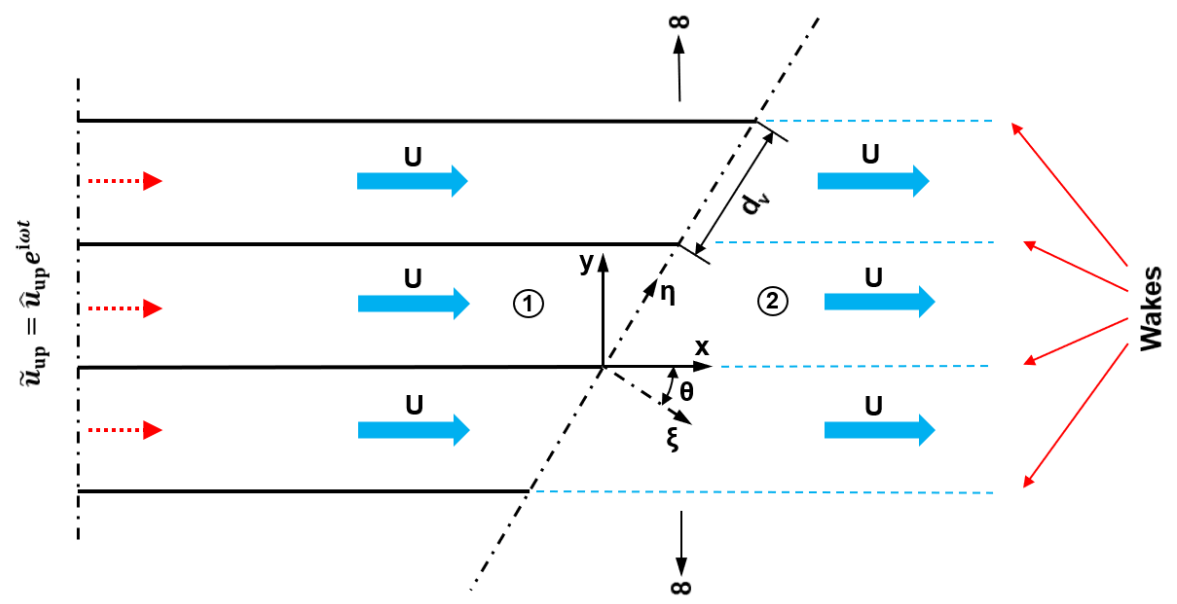

Figure 19: Incompressible oscillating flow with swirl vane wakes 


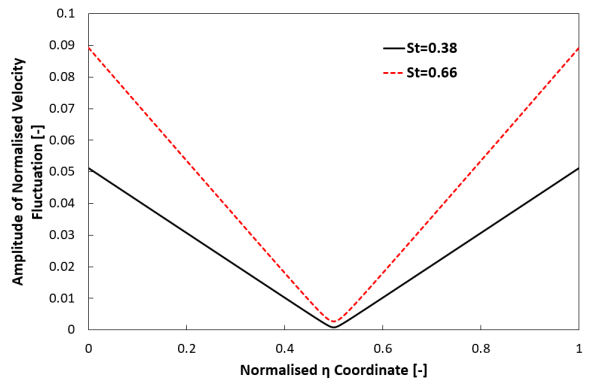

(a) Axial

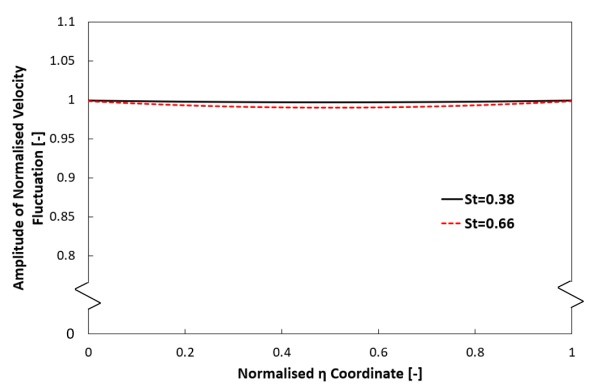

(b) Transverse

Figure 20: Amplitude of velocity fluctuation due to shed wakes 


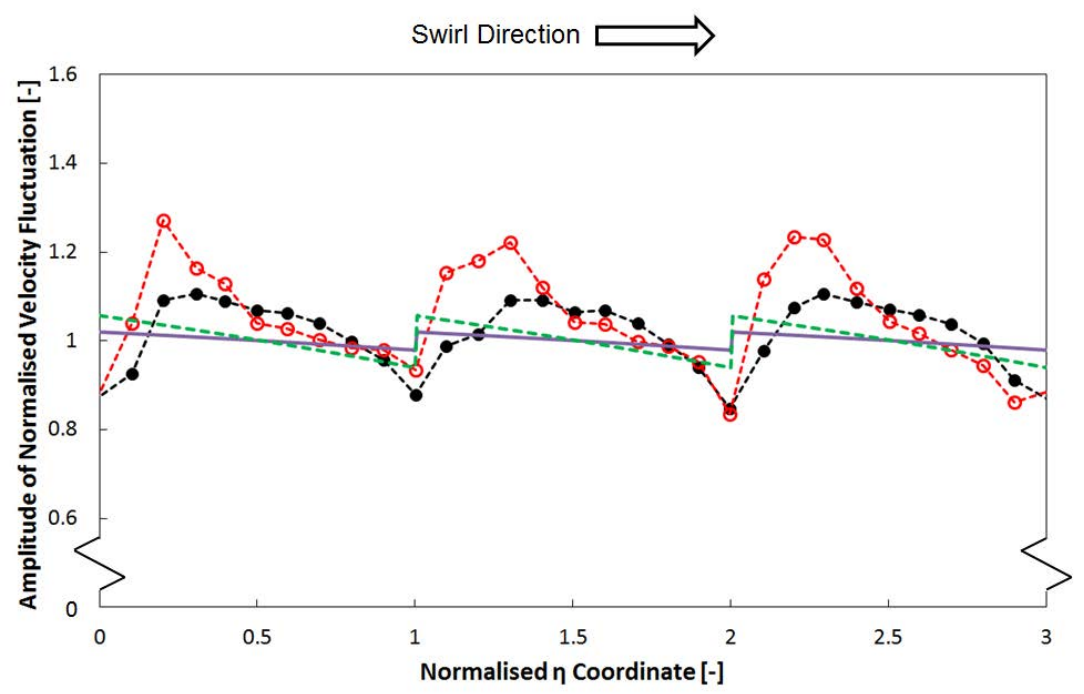

$-\bullet-\mathrm{St}=0.38$ (CFD) $-\Theta-\mathrm{St}=0.66$ (CFD) - $\mathrm{St}=0.38$ (Analytical) - - $\mathrm{St}=0.66$ (Analytical)

(a) Amplitude

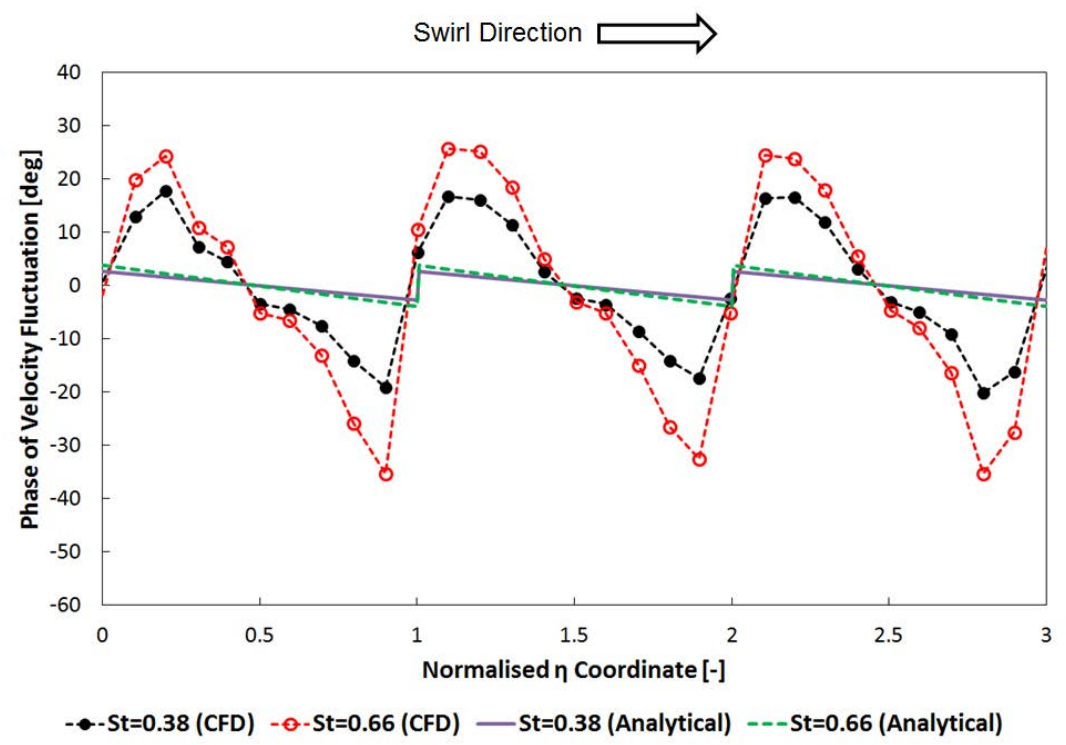

(b) Phase

Figure 21: Axial velocity fluctuation at passage exit 


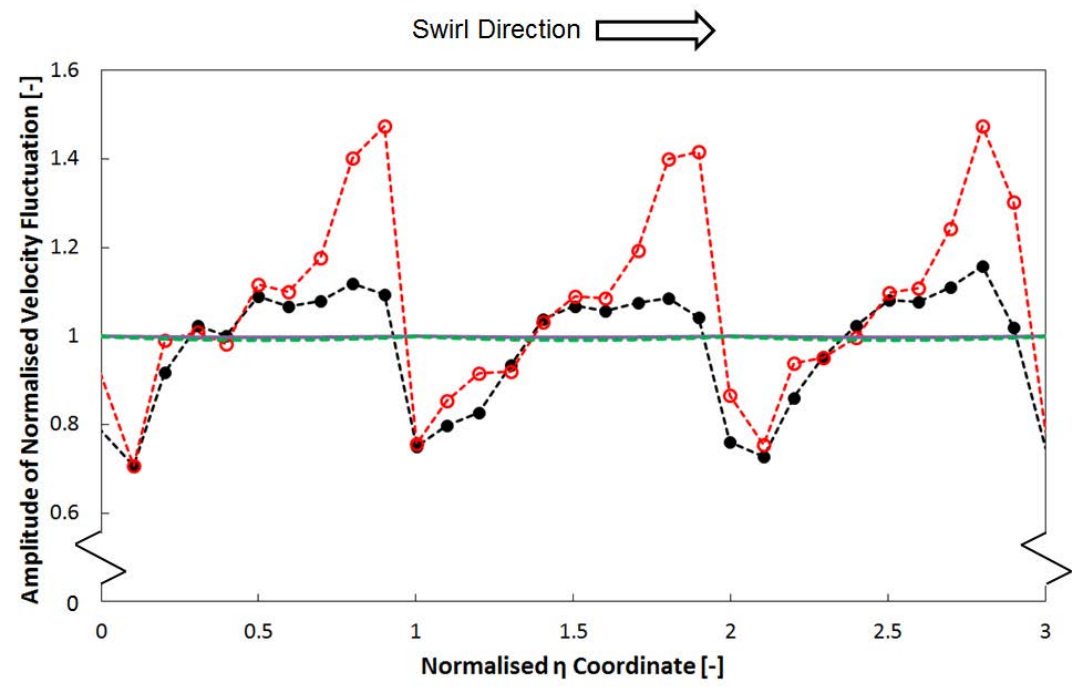

$-\bullet-\mathrm{St}=0.38$ (CFD) $-\Theta-\mathrm{St}=0.66$ (CFD) - $-\mathrm{St}=0.38$ (Analytical) - - $\mathrm{St}=0.66$ (Analytical)

(a) Amplitude

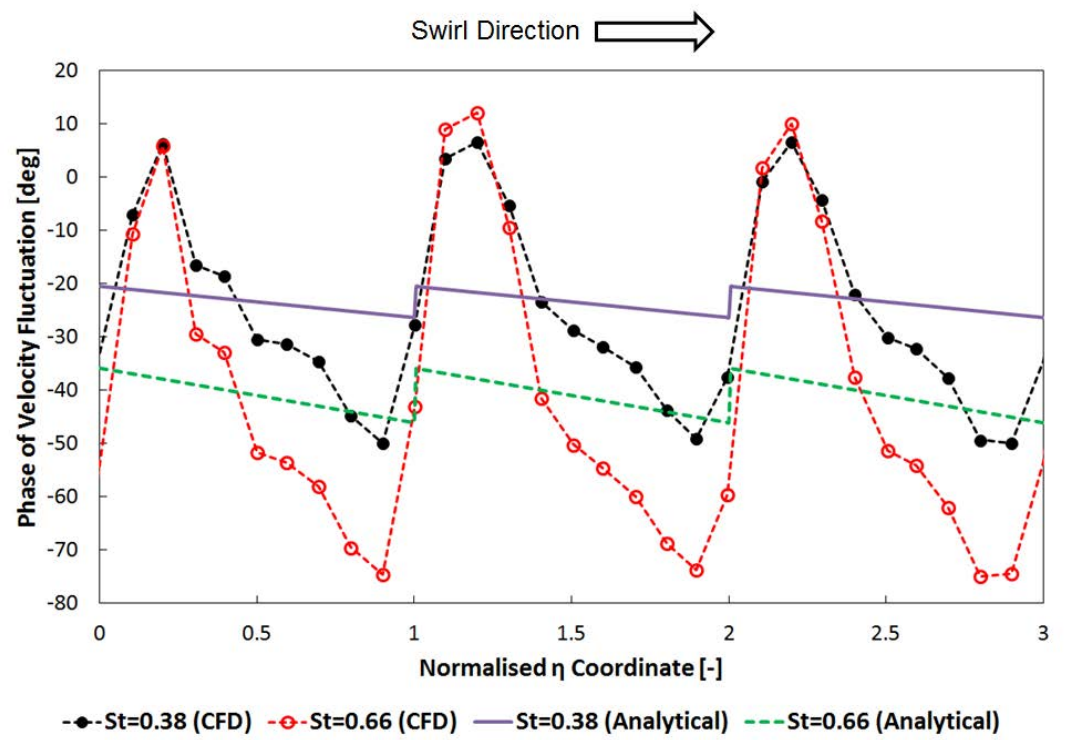

(b) Phase

Figure 22: Tangential velocity fluctuation at passage exit 


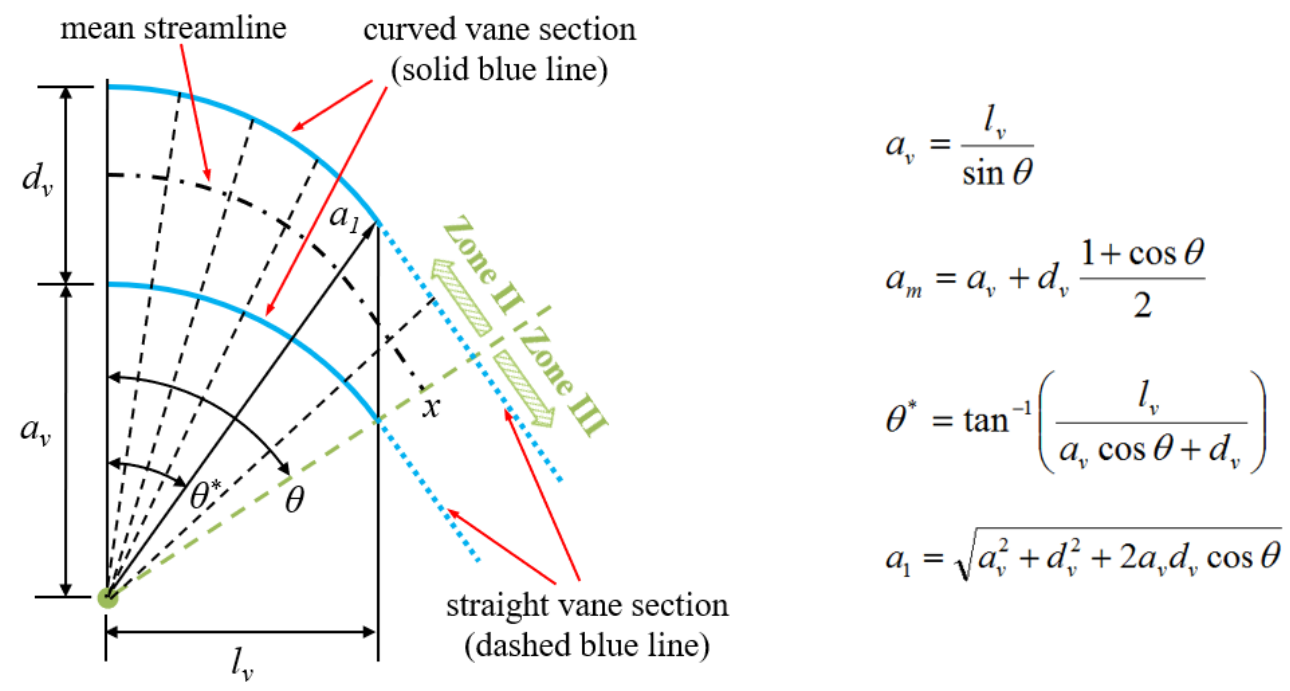

Figure A.1: Geometric relations of turning section of swirl vane

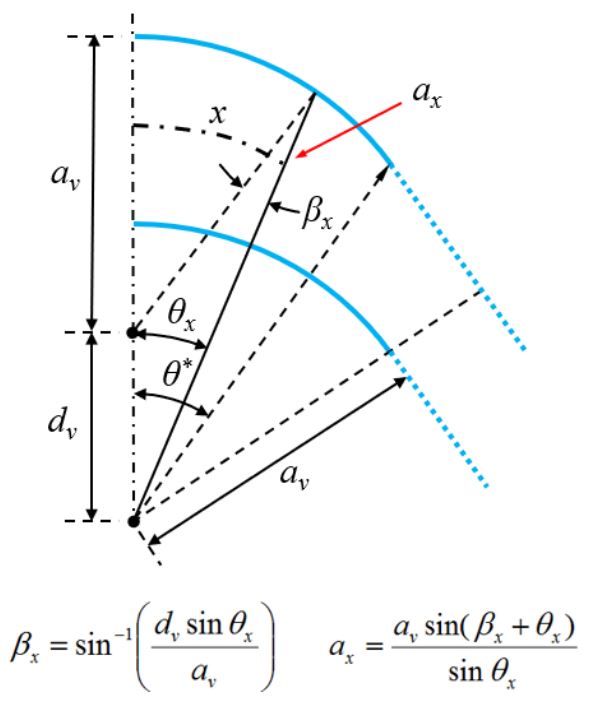

(a)

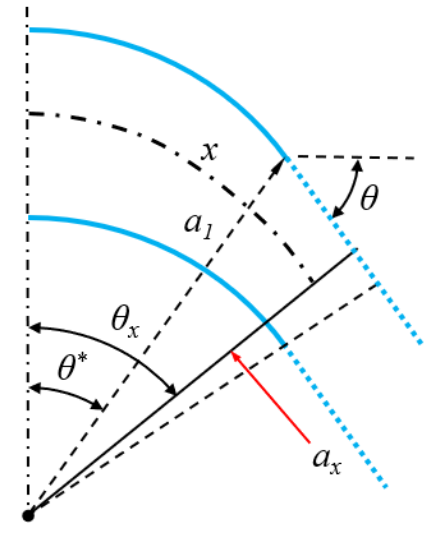

$$
a_{x}=\frac{a_{1} \cos \left(\theta-\theta^{*}\right)}{\cos \left(\theta_{x}-\theta\right)}
$$

(b)

Figure A.2: Geometric relation for determination of $A$ : (a) $\theta_{x}<\theta^{*}$, (b) $\theta_{x}>\theta^{*}$. 


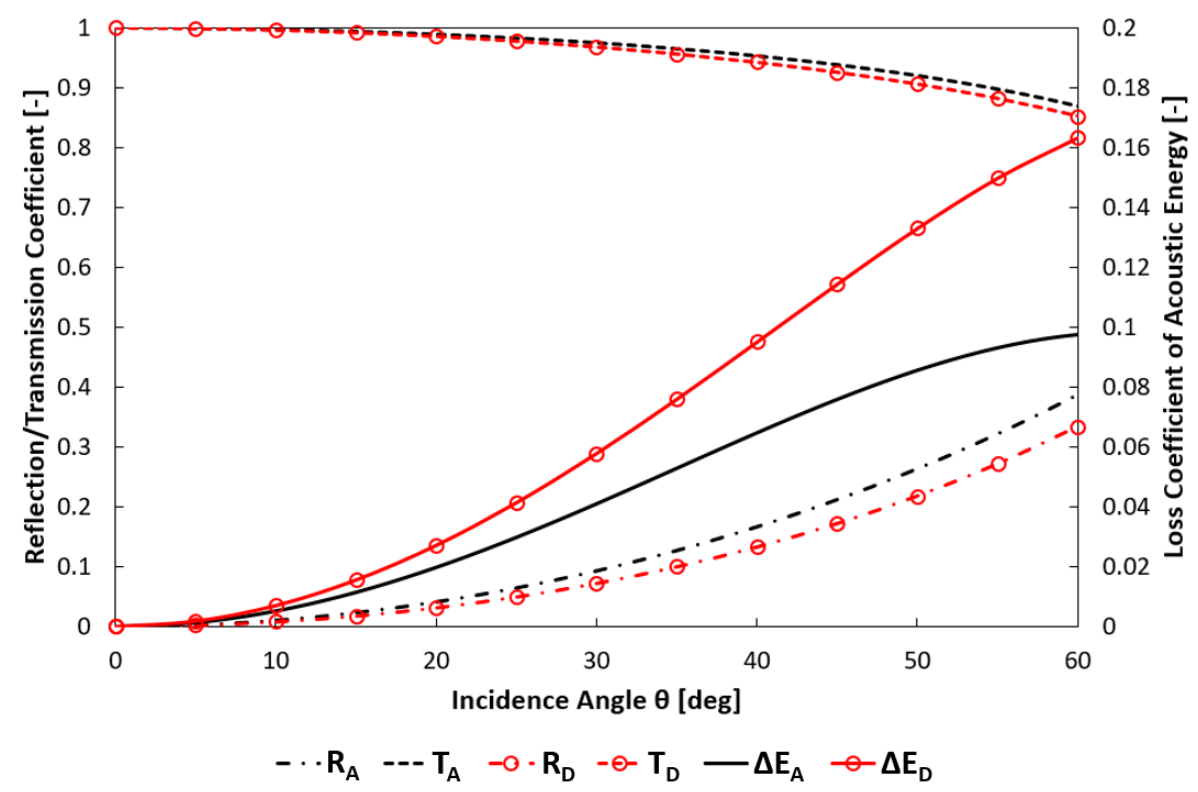

Figure D.1: Reflection and transmission coefficients as function of incidence angle

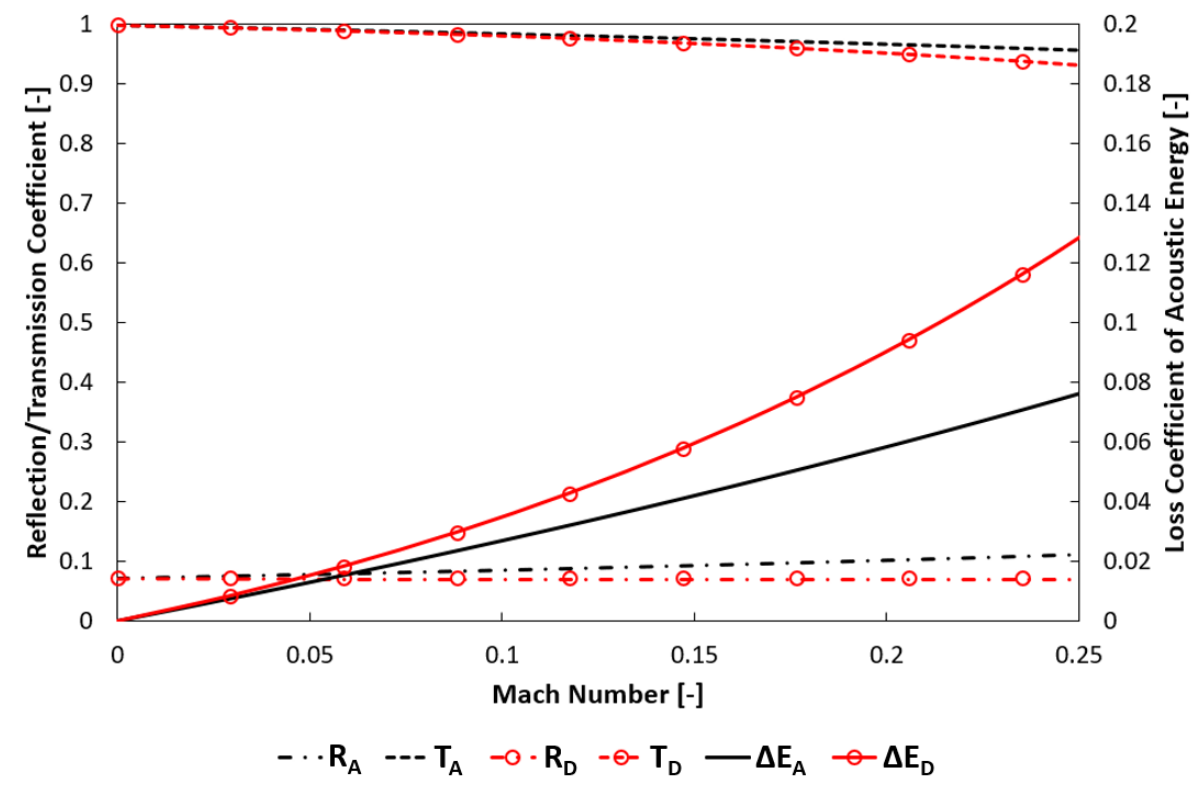

Figure D.2: Reflection and transmission coefficients as function of base flow Mach number 